\title{
THE INPACT OF HEAT-MOISTURE TREATMENT ON THE MOLECULAR STRUCTURE AND PHYSICOCHEMICAL PROPERTIES OF NORNAL AND WAXY POTATO STARCHES
}

\section{VARATHARAMAN VAMADEVAN}




$$
\text { . }
$$
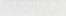


THE IMPACT OF HEAT-MOISTURE TREATMENT ON THE MOLECULAR STRUCTURE AND PHYSICOCHEMICAL PROPERTIES OF NORMAL AND WAXY POTATO

\section{STARCHES}

by

QVaratharajan Vamadevan

A thesis submitted to the School of Graduate Studies in partial fulfillment of the requirements for the degree of Master of Science

Department of Biochemistry

Memorial University of Newfoundland

November 2010

St. John's

Newfoundland and Labrador 


\begin{abstract}
\end{abstract}
Heat-moisture treatment (HMT) is a physical modification technique that modifies starch structure and properties without destroying its granular structure. HMT has been shown to cause starch chain interactions and crystallite disruption/reorientation within the amorphous and crystalline domains. However, the part played by amylose (AM) during HMT of starches is not properly understood. Furthermore, a systematic study has not been carried out to examine how variations in HMT temperatures influence molecular structure, physicochemical properties and digestibility of normal potato (NP) and waxy potato (WP) starches. Thus, the objective of this study was to determine changes to molecular structure, physicochemical properties and digestibility of NP and WP starches on HMT at different temperatures $\left(80,100,120\right.$ and $\left.130^{\circ} \mathrm{C}\right)$ for $16 \mathrm{~h}$ at $27 \%$ moisture. Structural changes on HMT were monitored by scanning electron microscopy (SEM), confocal laser scanning microscopy (CLSM), polarized light microscopy (PLM), wide angle X-ray scattering (WAXS), attenuated total reflection-Fourier transform infrared (ATR-FTIR) spectroscopy, fluorophore assisted capillary electrophoresis (FACE) and $\mathrm{K} / \mathrm{S}$ spectra. Changes to physicochemical properties on HMT were determined by granular swelling, amylose leaching, gelatinization parameters and pasting properties. Digestibility of normal and waxy potato starches before and after HMT by porcine pancreatic $\alpha$-amylase (PPA) was monitored by measuring initial velocity and by examination of the granular structure at different stages of hydrolysis by SEM, CLSM and PLM. The results showed that structural changes on HMT were influenced by differences in starch chain mobility at different temperatures of HMT. Starch chain mobility, in turn, was influenced by the interplay between the extent to which B-type crystallites were transformed into A+B-type crystallites, kinetic energy imparted to starch chains and amylose content. The main type of structural changes 
influencing physicochemical properties at different temperatures of HMT were starch chain interactions (at 80 and $100^{\circ} \mathrm{C}$ ), disruption of hydrogen bonds between amylose-amylopectin and amylopectin-amylopectin (at 120 and $130^{\circ} \mathrm{C}$ ), disorganization of amylopectin chains near the vicinity of the hilum (at 100,120 and $130^{\circ} \mathrm{C}$ ) and formation of interrupted helices (at $130^{\circ} \mathrm{C}$ ). The susceptibility of NP and WP starches towards $\alpha$-amylase decreased at $80^{\circ} \mathrm{C}$, but increased in the range 100 to $130^{\circ} \mathrm{C}$. NP and WP starches exhibited heterogeneity in degradation (NP>WP) in both their native and HMT states. 


\section{ACKNOWLEDGEMENTS}

I would like to express my sincere appreciation for the invaluable guidance and advice of my supervisor, Dr. R. Hoover. I also appreciate Dr. F. Shahidi and Dr. V. K. Booth for serving on my supervisory committee and for the time and effort that they spent on my behalf. I would like to acknowledge the Department of Biochemistry and the School of Graduate Studies for providing financial assistance. Financial support from Dr. R. Hoover is also acknowledged.

I would like to express my sincere thanks to Dr. K. Seetharaman, Dr. Q. Liu, Dr. T. Vasanthan, Dr. R. N. Chibbar, Ms. E. Donner, Dr. L. Jihong, K. K. M. Nantanga and Dr. S. Jaiswal for their help with some of the analytical techniques described in the thesis.

I wish to express my sincere thanks to Dr. A. Yethiraj (Department of Physics and Physical Oceanography) for assistance with confocal laser scanning and polarized microscopy. Thanks are also extended to my colleagues H. Lan, T. Hughes and A. Priyatharani.

Finally, I thank my wife, daughter, parents and friends for helping me in all possible ways to succeed. 


\section{TABLE OF CONTENTS}

\section{Page}

Abstract

Acknowledgements

iv

Table of Contents

v

List of Tables

ix

List of Figures

$\mathrm{x}$

List of Abbreviations $\quad$ xii

Chapter 1 Introduction 1

Chapter 2 Literature review 4

2.1 General 4

2.2 Starch granule morphology 4

2.2.1 Granule size and shape $\quad 4$

2.2.2 Pores, channels and cavities 6

2.3 Starch structure $\quad 10$

2.3.1 Major structural components $\quad 10$

2.3.1.1 Amylose 14

2.3.1.1.1 Fine structure of amylose $\quad 14$

2.3.1.1.2 Conformation of amylose $\quad 15$

2.3.1.1.3 Location of amylose $\quad 16$

2.3.1.1.4 Amylose-iodine inclusion complex 20

2.3.1.2 Amylopectin 23

2.3.1.2.1 Fine structure of amylopectin 23

2.3.1.2.2 Amylopectin iodine complex 26

2.3.2 Minor components $\quad 26$

2.3.2.1 Phosphorus $\quad 26$ 
2.3.2.2.1 Amylose-lipid inclusion complex 30

2.3.2.3 Protein $\quad 35$

2.3.3 Supramolecular structure $\quad 36$

2.3.3.1 Birefringence $\quad 40$

2.3.3.2 Starch crystallinity $\quad 42$

2.3.3.3 Degree of crystallinity 51

2.4 Starch properties $\quad 52$

2.4.1 Granular swelling $\quad 52$

2.4.2 Amylose leaching $\quad 54$

2.4.3 Gelatinization $\quad 55$

2.4.4 Pasting properties 61

2.4.5 Retrogradation $\quad 62$

2.4.6 Acid hydrolysis $\quad 63$

2.4.7 Enzyme hydrolysis of starch by $\alpha$-amylase $\quad 64$

2.4.7.1 Classification $\quad 64$

2.4.7.2 Mechanism for enzymatic hydrolysis 65

2.4.7.2.1 Double displacement mechanism 67

2.4.7.3 Mode of action of $\alpha$-amylase $\quad 69$

2.4.7.4 Stages of enzymatic reaction $\quad 69$

2.4.7.5 Enzyme hydrolysis of native starches $\quad 70$

2.5 Heat-moisture treatment (HMT) $\quad 74$

2.5.1 Impact of HMT on granule morphology 76

2.5.2 Impact of HMT on X-ray pattern and crystallinity 77

2.5.3 Impact of HMT on granular swelling and amylose leaching $\quad 79$

2.5.4 Impact of HMT on gelatinization parameters $\quad 80$

2.5.5 Impact of HMT on pasting properties $\quad 82$

2.5.6 Impact of HMT on enzyme hydrolysis 83

2.5.7 Uses of HMT starches $\quad 84$ 
$\begin{array}{ll}3.1 \text { Materials } & 87\end{array}$

$\begin{array}{ll}3.2 \text { Methods } & 87\end{array}$

$\begin{array}{ll}3.2 .1 \text { Chemical composition of starch } & 87\end{array}$

$\begin{array}{ll}\text { 3.2.1.1 Moisture content } & 87\end{array}$

3.2.1.2 Nitrogen content $\quad 87$

3.2.1.3 Phosphorus content $\quad 87$

3.2.1.4 Ash content $\quad 88$

3.2.1.5 Lipid content $\quad 89$

$\begin{array}{ll}3.2 .1 .5 .1 & \text { Surface lipid }\end{array}$

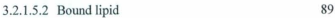

3.2.1.6 Amylose content $\quad 90$

3.2.1.6.1 Apparent amylose content $\quad 90$

3.2.1.6.2 Total amylose content 91

3.2.2 Heat-moisture treatment $\quad 91$

3.2.3 Polarized microscopy 92

3.2.4 Scanning electron microscopy 92

3.2.5 Confocal laser scanning microscopy $\quad 92$

3.2.6 Iodine binding 93

3.2.7 Fluorophore-assisted capillary electrophoresis 94

3.2.8 Attenuated total reflectance Fourier transform infrared spectroscopy 96

3.2.9 Wide angle X-ray diffraction 96

3.2.10 Amylose leaching $\quad 97$

3.2.11 Swelling factor $\quad 97$

3.2.12 Differential scanning calorimetry 98

3.2.13 Rapid Visco Analyser $\quad 99$

3.2.14 Enzymatic hydrolysis $\quad 99$

$\begin{array}{ll}3.2 .15 \text { Statistical analysis } & 101\end{array}$

$\begin{array}{lll}\text { Chapter } 4 \text { Results and discussion } & 102\end{array}$

$\begin{array}{ll}4.1 \text { Composition } & 102\end{array}$ 
4.2 Morphology 102

4.2.1 Scanning electron microscopy 102

4.2.2 Confocal laser scanning microscopy 105

4.2.2.1 Phosphate distribution 105

4.2.2.2 Growth rings and amylose distribution 107

4.2.3 Polarized and bright field microscopy 109

4.3 Amylopectin chain length distribution $\quad 111$

4.4 X-ray diffraction 111

4.5 ATR-FTIR spectroscopy $\quad 116$

4.6 Amylose leaching $\quad 118$

4.7 Swelling factor $\quad 120$

$4.8 \mathrm{~K} / \mathrm{S}$ spectral analysis $\quad 123$

4.9 Gelatinization characteristics $\quad 128$

4.10Pasting properties 131

4.11Enzyme Hydrolysis 135

Chapter 5 Summary, conclusions and directions for future research 146

$\begin{array}{lr}\text { References } & 149\end{array}$

$\begin{array}{ll}\text { Publications } & 194\end{array}$

$\begin{array}{ll}\text { Scholarships and awards } & 195\end{array}$

$\begin{array}{ll}\text { Appendices } & 196\end{array}$

$\begin{array}{ll}\text { Appendix } 1 & 197\end{array}$

$\begin{array}{ll}\text { Appendix } 2 & 198\end{array}$

$\begin{array}{lr}\text { Appendix } 3 & 199\end{array}$

$\begin{array}{ll}\text { Appendix } 4 & 200\end{array}$ 


\section{LIST OF TABLES}

Table

2.1 Food and non-food applications of starches

2.2 Size and shape of starch granules from different botanical origins 7

2.3 Proximate composition of starches from different botanical origins 13

2.4 Double helical content and crystallinity of starches 43

4.1 Chemical composition (\%) of normal and waxy potato starches 103

4.2 Amylopectin chain length distribution of native and HMT normal and waxy $\quad 112$ potato starches

4.3 Peak height $(\mathrm{PH})$ and full width at half-maximum peak height (FWHM) of 115 $\mathrm{X}$-ray reflections

4.4 ATR-FTIR spectral data for native and HMT starches

4.5 Gelatinization parameters of native and HMT normal and waxy potato starches 130

4.6 RVA parameters of native and HMT normal and waxy potato starches 134 


\section{LIST OF FIGURES}

Figure

2.1 Schematic diagram of amylose and amylopectin

2.2 Models proposed for amylose in aqueous solution

2.3 Model proposed for the co-crystallization of amylose with amylopectin

2.4 Schematic illustration of amylose-iodine inclusion complex

2.5 A cluster model of amylopectin

2.6 Schematic illustration of amylose-lipid complex

$2.7 \quad{ }^{13} \mathrm{C}$-CP-MAS NMR spectra of V-amylose

2.8 DSC thermal curve of a cereal starch showing the different melting transitions and the corresponding structural domains

2.9 Schematic representation of the starch granule

2.10 Localization of amylose chains in amylopectin clusters

2.11 Schematic model of the organization of a starch granule

2.12 X-ray diffraction patterns of A-, B- and C-type starches

2.13 V-type X-ray diffraction pattern of amylose-lipid complex

2.14 Arrangement of double helices A-type and B-type crystallites in starch granules

2.15 Structure of A- and B-type unit cells

2.16 Proposed models for A-type and B-type amylopectin branching patterns

2.17 A proposed model for gelatinization mechanism

2.18 Schematic representation of the action of different amylolytic enzymes on starch 66 (amylopectin) polymers

2.19 Proposed mechanisms involved in the hydrolysis of glycosidic linkages

2.20 An energy profile ( $\Delta \mathrm{H} \mathrm{kcal} / \mathrm{mole}$ ) for the inter-conversion of the chair to a 'half chair' conformation 
2.21 A model for the polymorphic transition from B-type to A-type unit cell in the 78 solid state

3.1 Labelling reaction of glucose with APTS

4.1 Scanning electron microscopy of native normal and waxy potato starches

4.2 Confocal laser scanning microscopy of Pro-Q Diamond stained granules of 106 native and porcine pancreatic $\alpha$-amylase hydrolysed normal and waxy potato starches

4.3 Confocal laser scanning microscopy of APTS stained granules of native and 108 HMT normal and waxy potato starches

4.4 Polarized light microscopy of native and HMT normal potato and waxy potato starches

4.5 X-ray patterns of native and HMT normal potato and waxy potato starches

4.6 Amylose leaching of native and HMT normal potato starches

4.7 Swelling factor of native and HMT normal and waxy potato starches

4.8 Schematic model for structural changes on HMT at different temperatures and their impact on iodine binding

4.9 Reflectance spectra of native and HMT normal and waxy potato starches

4.10 RVA profiles of native and HMT normal and waxy potato starches

4.11 Hydrolysis of native and HMT normal and waxy potato starches by porcine pancreatic $\alpha$-amylase

4.12 Microscopy of porcine pancreatic $\alpha$-amylase hydrolysed residues of native and HMT normal and waxy potato starches

4.13 Confocal laser scanning microscopy of APTS stained porcine pancreatic $\alpha$-amylase hydrolysed native and HMT normal and waxy potato starches

4.14 Scanning electron microscopy of porcine pancreatic $\alpha$-amylase hydrolysed native and HMT normal and waxy potato starches 


\section{LIST OF ABBREVIATIONS}

AFM

$\mathrm{AM}$

AML

AMP

APTS

ATR-FTIR

$\mathrm{a}_{w}$

BFM

${ }^{13} \mathrm{C}$-CPMAS-NMR

CL

CLSM

Cps

$\mathrm{db}$

DMSO

dp

DSC

FACE

HMT

MALDI-TOF MS

NP

PLM
Atomic force microscopy

Amylose

Amylose leaching

Amylopectin

Aminopyrene trisulfonic acid

Attenuated total reflectance Fourier transform infrared

spectroscopy

Water activity

Bright field microscopy

Cross-polarization magic angle spinning carbon-13 nuclear magnetic resonance

Chain length

Confocal laser scanning microscopy

Counts per second

Dry basis

Dimethyl sulfoxide

Degree of polymerization

Differential scanning calorimetry

Fluorophore-assisted capillary-electrophoresis

Heat-moisture treatment

Matrix assisted laser desorption ionization - time of flight mass spectroscopy

Normal potato

Polarized light microscopy 
PPA

RDS

RS

RVA

SCLCP

SDS

SEM

SF

TEM

$\mathrm{Tg}$

Tm

XRD

WP
Porcine pancreatic $\alpha$-amylase

Rapidly digestible starch

Resistant starch

Rapid Visco Analyser

Side chain liquid crystalline polymer

Slowly digestible starch

Scanning electron microscopy

Swelling factor

Transmission electron microscopy

Glass transition temperature

Melting temperature

X-ray diffraction

Waxy potato 


\section{CHAPTER 1}

\section{INTRODUCTION}

Starch is an important functional ingredient in many food products. However, native starches usually do not meet industrial needs in which starch should be able to withstand low acidity, high temperatures and high shear forces. Consequently, starches have been modified physically and chemically in order to extend the range of starch applications in the food and non-food sectors (Hoover, 2010). Heat-moisture treatment (HMT) is a physical modification technique wherein starch granules at low moisture levels $\left(<5 \% \mathrm{H}_{2} \mathrm{O}, \mathrm{w} / \mathrm{w}\right)$ are heated at a temperature above the glass transition temperature $(\mathrm{Tg})$ but below the gelatinization temperature for a fixed period of time (15 min to $16 \mathrm{~h}$ ). HMT has been shown to change starch properties by facilitating starch chain interactions within the amorphous and crystalline domains and/or by disrupting starch crystallites, the extent of these changes being influenced by starch composition, organization of amylose and amylopectin within the native granules, and by the conditions (temperature, moisture, time) prevailing during HMT (Hoover, 2010). Regardless of the origin of the starch, HMT increases the gelatinisation transition temperature, widens the gelatinisation temperature range, decreases granular swelling and amylose leaching, and increases thermal stability. Depending on the starch source, changes to the $\mathrm{X}$-ray pattern $(\mathrm{B}$ to $\mathrm{A}+\mathrm{B})$, formation of amylose-lipid complexes, disruption of crystallinity, and an increase or decrease in enzyme susceptibility have also been shown to occur on HMT (Gunaratne and Hoover, 2002; Hoover and Vasanthan, 1994a).

Most studies have been on the impact of HMT on the thermal properties, crystallinity and pasting properties of normal starches. Some researchers (Hoover and Manuel, 1996; Kweon et al., 2000; 
Zavareze et al., 2010) have attempted to explain the impact of HMT on the structure and physicochemical properties of starches varying in amylose content. However, the part played by amylose during HMT of starches is still not properly understood. Unlike in cereal starches, amylose has been shown to be co-crystallized with amylopectin in normal potato starch (Hoover and Vasanthan, 1994a; Saibene et al., 2008; Zobel, 1988a). Therefore, it is hypothesized that amylose may have a significant impact on the extent to which double helical chains forming the crystalline lamellae of potato amylopectin move and rearrange during HMT.

HMT has been conducted mainly at a fixed temperature and moisture content for periods varying from $15 \mathrm{~min}$ to $24 \mathrm{~h}$. No attempt has been made to investigate the influence of varying temperature and moisture content during HMT on starch structure and properties. Furthermore, the impact of HMT on starch digestibility has received only scant attention. Kweon et al. (2000) and Zavareze et al. (2010) studied the influence of moisture content during HMT on the digestibility of normal, waxy and high amylose maize $\left(110^{\circ} \mathrm{C} / 16 \mathrm{~h}\right)$ and rice $\left(110^{\circ} \mathrm{C} / 1 \mathrm{~h}\right)$ starches, respectively. Digestibility of HMT maize starches by porcine pancreatic $\alpha$-amylase (PPA) was shown to decrease in the moisture range of (15-21\%) but to increase at $27 \%$ moisture (Kweon et al., 2000). However, Zavareze et al. (2010) reported increased PPA hydrolysis with an increase in moisture content (15 to 25\%) in HMT rice starches. Hoover and Manuel (1996) reported decreased PPA hydrolysis on HMT $\left(100^{\circ} \mathrm{C}, 30 \%\right.$ moisture, $\left.16 \mathrm{~h}\right)$ of normal, waxy and high amylose maize starches. In pulse (Hoover and Manuel, 1995) and tuber (Gunaratne and Hoover, 2002) starches, PPA hydrolysis was shown to increase on $\mathrm{HMT}\left(100^{\circ} \mathrm{C}, 30 \%\right.$ moisture, $16 \mathrm{~h})$. The results from all of the above studies showed that the impact of HMT on PPA hydrolysis is influenced by starch moisture content and by structural (crystallite disruption, 
change in polymorphic form, crystallite reorientation, amylose-lipid interaction, starch chain interaction) and morphological (formation of fissures and cracks on the granule surface) changes on HMT. However, no systematic study has been carried out to investigate the susceptibility to PPA of starches subjected to HMT at different temperatures but at the same moisture level and time period of heat treatment. It is hypothesised, that under the above conditions, structural changes within the amorphous and crystalline domains of starch granules will vary at different temperatures of HMT, due to differences in starch chain mobility. Consequently, this would influence the accessibility of PPA to the amorphous and crystalline domains of the starch granule. Thus, a comparison of the action pattern of PPA on native normal and waxy potato starches with those of their HMT counterparts may provide deep insight into the influence of starch structure on the rate and extent of a-amylolysis.

The objectives of this study were as follows: 1) to determine the structural and property changes of normal and waxy potato starches subjected to heat-moisture treatment at $80,100,120$ and $130^{\circ} \mathrm{C}$ at $27 \%$ moisture for $16 \mathrm{~h}$ and 2 ) to determine the impact of structural changes on the susceptibility (monitored by determination of the rate and extent of hydrolysis and degradation pattern) of normal and waxy potato starches to PPA hydrolysis. 


\section{CHAPTER 2}

\section{LITERATURE REVIEW}

\subsection{General}

Starch is a natural biopolymer produced by many plants as a source of stored energy. It is the second most abundant biomass material in nature. Starch is stored in seeds, tubers, roots, stems, leaves, fruits and pollen. The most important sources of starch are cereal grains (e.g., rice, maize, wheat, barley, sorghum and others), tubers (e.g., potato, sweet potato, yam), storage roots (e.g., cassava, taro) and seeds of beans and peas. Worldwide, the main sources of starch are maize $(82 \%)$, wheat $(8 \%)$, potatoes $(5 \%)$ and cassava ( $5 \%$ ) (Le Corre et al., 2010$)$.

The unique physical properties and behaviour of starch makes it an important functional ingredient in many food products. Starch contributes $50-70 \%$ of the energy in the human diet (Copeland et al., 2009). In addition to being an important source of energy in the human diet, starch is also utilized in many different industrial processes because it is abundant, renewable, biodegradable and safe (Table 2.1). However, native starches from various plant species have poor functionality, and are thus not directly suitable for food applications. Consequently, to meet industrial demands, modification of starch is often needed (Hoover, 2010).

\subsection{Starch granule morphology}

\subsubsection{Granule size and shape}

Starch occurs in granules that are relatively dense, and the granules from different botanical sources vary in their size and shape (Jane et al., 1994; Svegmark and Hermansson, 1993). 
Table 2.1 Food and non-food applications of starches

\begin{tabular}{|c|c|}
\hline Application area & Uses of native and modified starches \\
\hline Food & $\begin{array}{l}\text { Bakery products, batters and breadings, beverages, confectionery, gravies } \\
\text { and creams, syrups and desserts, fat mimetics, yoghurts, cheese and } \\
\text { imitation cheese, meat binders, cereals and snacks, puddings, instant meals, } \\
\text { dressings, soups, sauces, viscosity modifiers, glazing agents, stabilizing } \\
\text { agents }\end{array}$ \\
\hline Functional foods & $\begin{array}{l}\text { Ingredient (e.g., slowly digestible starch [SDS], resistant starch [RS]), } \\
\text { encapsulant }\end{array}$ \\
\hline Pharmaceutical & Diluent, binder, drug delivery, dusting agent, pill coating \\
\hline Medical & $\begin{array}{l}\text { Plasma extender/replacement (hydroxyethyl starch plasma), organ } \\
\text { preservation, absorbent sanitary products, plasters and dressings }\end{array}$ \\
\hline Biotechnology & Substrate for growing microorganisms \\
\hline Fuel & Bioethanol (e.g., corn starch) \\
\hline Adhesive & $\begin{array}{l}\text { Hot-melt glues, stamps, book binding, envelopes, labels, wood adhesives, } \\
\text { lamination, paper bags }\end{array}$ \\
\hline Agrochemical & Mulches, pesticide delivery, seed coatings \\
\hline Cosmetics & Dusting powder, make-up, soap filler/extender, face cream, tooth paste \\
\hline Detergent & Bio-surfactants, builders, co-builders, bleaching agents, bleaching activators \\
\hline Paper & $\begin{array}{l}\text { Binding, internal sizing, surface sizing, filler retention, paper coating, } \\
\text { carbonless paper stilt material, disposable diapers, feminine products }\end{array}$ \\
\hline Plastics & Biodegradable filler \\
\hline $\begin{array}{l}\text { Biologically- } \\
\text { degradable materials }\end{array}$ & Biodegradable plastic film (e.g., food packaging materials) \\
\hline Textiles & Warp sizing, fabric finishing and printing, fire resistance \\
\hline Ceramics & Filler \\
\hline Metals & Foundry core binder, sintered metal additive, sand casting binder \\
\hline Construction & $\begin{array}{l}\text { Concrete block binder, paint filler, plywood/chipboard adhesive, asbestos, } \\
\text { clay/limestone binder, fire resistant wallboard, gypsum board binder }\end{array}$ \\
\hline Purification & Flocculent for wastewater treatment \\
\hline Mining & $\begin{array}{l}\text { Viscosity modifier (oil), ore flotation, ore sedimentation, oil well drilling } \\
\text { muds }\end{array}$ \\
\hline $\begin{array}{l}\text { Explosives } \\
\text { Miscellaneot }\end{array}$ & $\begin{array}{l}\text { Wide range binding agent, match-head binder } \\
\text { Dry cell batteries, printed circuit boards, leather finishing }\end{array}$ \\
\hline Miscellaneo & Dry cell batteries, printed circuit boards, leather fimisning \\
\hline
\end{tabular}


The morphology of starch granules depends on the biochemistry of the chloroplast or amyloplast, as well as the physiology of the plant (Badenhuizen, 1969). In some sources, the age of the starch could cause differences in the size of the starch granules (French, 1984; Shannon and Garwood, 1984). Diameter of starch granules varies from less than $1 \mu \mathrm{m}$ (e.g., amaranth, small pigweed) to more than $100 \mu \mathrm{m}$ (e.g., canna) (Hoover, 2001; Jane, 2009; Pérez and Bertoft, 2010). Light microscopy, scanning electron microscopy (SEM), transmission electron microscopy (TEM) and atomic force microscopy (AFM) are widely used to characterize the size and shape of starch granules (Baker et al., 2001; Baldwin et al., 1997; Gallant et al., 1992; Glaring et al., 2006; Juszczak et al., 2003; Ridout et al., 2002; Sujka and Jamroz, 2009).

Tuber starches are generally large, ellipsoid or spherical with apparent shells around an eccentric hilum. Cereal starches are generally small and polyhedric with a concentric hilum (Gallant $e t$ al., 1992; Hoover, 2001; Tester and Karkalas, 2002). Wheat, triticale, barley and rye starches have

bimodal size (large A- and small B-type) distributions (Peng et al., 1999; Stoddard, 1999). High amylose maize starch exhibits filamentous granules (budlike protrusions) (Jane, 2009). Legume starches are kidney-like or ovoid with well-defined shells centred along an elongated hilum (Hoover et al., 2009). The general characteristics of starch granules are presented in Table 2.2.

\subsubsection{Pores, channels and eavities}

Surface pores, cavities and channels are important characteristic features of starch granules. They are large enough that they can be seen by SEM (Fannon et al., 1992, 2004; Sujka and Jamroz, 2007). Pores have been observed on the granule surface of the following starches: corn, 
Table 2.2 Size and shape of starch granules from different botanical origins

\begin{tabular}{|c|c|c|c|}
\hline Starch source & Shape & Size $(\mu \mathrm{m})$ & Source of information \\
\hline \multicolumn{4}{|l|}{ Potato } \\
\hline Normal & Oval, spherical, & $5-100$ & Tester and Karkalas (2002) \\
\hline Waxy & Round, oval & $5-100$ & ELIANETM $(2006)^{\circ}$ \\
\hline High amylose & Rough & - & Glaring et al., (2006) \\
\hline Sweet potato & Round, oval, polygonal & $2-42$ & Lim et al. (1994) \\
\hline Yam (D. alata) & Oblong, oval & $12-100$ & Gunaratne and Hoover (2002) \\
\hline Cassava & Spherical, truncated, round & $7-25$ & Mishra and Raj (2006) \\
\hline Canna & Elliptical & $6-79$ & Peroni et al. (2006) \\
\hline Arrowroot & Round, oval & $9-42$ & Peroni et al. (2006) \\
\hline Ginger & Polygonal & 3-34 & Peroni et al. (2006) \\
\hline Barley & Round, elliptical & $2-25$ & Davis et al. (2003) \\
\hline \multicolumn{4}{|l|}{ Corn } \\
\hline Normal & Spherical, polyhedral & $2-30$ & Tester and Karkalas (2002) \\
\hline Waxy & Spherical, polyhedral & $2-30$ & Tester and Karkalas (2002) \\
\hline High amylose & Irregular & $2-30$ & Tester and Karkalas (2002) \\
\hline Oat & Polyhedral & $\begin{array}{l}3-10 \text { (single), } \\
80 \text { (compound) }\end{array}$ & Tester and Karkalas (2002) \\
\hline Rice & Polyhedral & $\begin{array}{l}3-8 \text { ( single), } \\
150 \text { (compound) }\end{array}$ & Tester and Karkalas (2002) \\
\hline Sago & Oval & $20-40$ & Tester and Karkalas (2002) \\
\hline Sorghum & Spherical & $5-20$ & Tester and Karkalas (2002) \\
\hline \multirow[t]{2}{*}{ Rye } & Lenticular (A-type) & $10-40$ & Tester and Karkalas (2002) \\
\hline & Spherical (B-type) & $5-10$ & Tester and Karkalas (2002) \\
\hline Triticale & Spherical & $1-30$ & Tester and Karkalas (2002) \\
\hline \multirow[t]{2}{*}{ Wheat } & Lenticular (A-type) & $15-35$ & Tester and Karkalas (2002) \\
\hline & Spherical (B-type) & $2-10$ & Tester and Karkalas (2002) \\
\hline Lentil & Oval, round, ellipsoidal & $15-30$ & Hoover, et al. (2009) \\
\hline Smooth pea & $\begin{array}{l}\text { Oval, round, irregular, } \\
\text { rentiform }\end{array}$ & $2-40$ & Hoover et al. (2009) \\
\hline Wrinkled pea & Round & $17-30$ & Hoover et al. (2009) \\
\hline
\end{tabular}

${ }^{*}$ ELIANE $^{\text {TM }}$ is a waxy potato starch marketed by the starch company AVEBE 
sorghum, millet (over the entire granule surface), wheat, rye, and barley (along the equatorial groove of large granules). Granules of potato, tapioca, rice, oat, canna, and arrowroot appear to have no pores, but there is disagreement about the presence of pores on the surface of potato starch granules (Fannon et al., 1992). The occurrence of pores on the potato starch granular surface was reported by Sterling (1973). AFM images showed depressions and protrusions (ranging up to $1 \mu \mathrm{m}$ in size) on the surface of potato and tapioca starch granules (Juszczak et al., 2003). Singh et al. (2006) have also reported the presence of some small nodules or protuberances, and surface fragmentation, on potato starch granules. This suggests that the structure of starch granules is more complicated than can be seen in photographs made by conventional SEM techniques. Li et al. (2007) have shown that pores on maize starch granules develop during a late maturation stage, i.e. 30 days after pollination (DAP). Starch granules isolated from maize kernels harvested 30 DAP showed few or no pores, whereas those from kernels harvested 45 DAP (fully matured and dried in the field) showed a large number of pores (Li et al., 2007). Apart from surface pores, Huber and BeMiller (1997) observed central cavities in normal maize, waxy maize and sorghum starch granules and they proved that the cavities were not formed by drying but could be enlarged by this process.

The pores were shown to be openings to channels $(5-400 \mathrm{~nm}$ in diameter) connecting an internal cavity at the granule hilum to the external surface (Fannon et al., 1993; Huber and BeMiller, 1997, 2000). It is still unclear how pores and channels are formed in certain starch granules. Jane et al. (1993) isolated starch granules from different parts of the corn kernel. They reported that starch granules near to the germ contained more pores than granules from other parts of the kernel, and suggested that this was a consequence of active in situ amylase(s). Fannon et al. 
(2004) hypothesized that amyloplasts of maize and sorghum endosperm tissue contained microtubules that radiated from the locus of the hilum outward to the plastid periphery and that the granule develops around the radially oriented microtubules, which become the granule channels that terminate in openings to the outside surface of the granule. Benmoussa et al. (2010) also believed that the channels in maize starch granules are remnants of amyloplast microtubules. Microtubules may serve at least two purposes in amyloplasts and granules in which they occur: (1) they may facilitate starch polymer and granule biosynthesis and, (2) they may function to provide variation in the rate of granule breakdown during seed germination (Benmoussa et al., 2010).

The channels of corn starch granules have been shown to be lined with proteins and phospholipids (Benmoussa et al., 2010; Fannon et al., 2004; Gray and BeMiller, 2004; Han et al., 2005; Han and Hamaker, 2002). Han et al. (2005) found that Bt1 protein, the major translocator of ADP-glucose (Cao and Shannon, 1997; Shannon et al., 1998) into the amyloplast for starch synthesis, was missing after digestion of starch granules with thermolysin and concluded that Bt1 protein is present in channels. Further investigations of the nature of channels were done by Gray (2003), who found that the binding of silver ions was completely removed only after treatment with both thermolysin and phospholipase $\mathrm{C}$, and not by treatment with either one alone, indicating the presence of both proteins and phospholipids in channels. Subsequently, phospholipid was identified by matrix assisted laser desorption ionization-time-offlight mass spectroscopy (MALDI-TOF MS) as lysophosphatidyl choline with either palmitic acid [16:0] or linoleic acid [18:2, n-6] fatty acid moieties (Lee and BeMiller, 2008). 
The number of individual channels in corn starch granules varies from granule to granule, and the average number of channels per granule in a population of granules is a function of the genetic makeup of the parent plant (Fannon et al., 2004; Widya et al., 2010). In wheat starch, Atype granules contain two types of channels: larger channels located on the equatorial groove and smaller channels located throughout the granule. B-type wheat starch granules contain only one type of channel: large, void-like channels that are less defined than the channels of A-type granules (Kim and Huber, 2008). The presence of pores, channels and cavities increases the granule surface which is potentially available for chemical and enzymatic reactions (Sujka and Jamroz, 2007 and 2009).

\subsection{Starch structure}

Starch is a semicrystalline biopolymer, which displays a hierarchical structural periodicity. It is considered to be structured on five different length scales: the whole granule architecture (1-100 $\mu \mathrm{m})$, growth rings (120-400 nm), blocklets (20-500 nm), amorphous and crystalline lamellas ( 9 $\mathrm{nm}$ ) and amylopectin and amylose chains (0.1-1.0 nm) (Copeland et al., 2009; Perez and Bertoft, 2010; Tester et al., 2004; Vandeputte and Delcour, 2004).

\subsubsection{Major structural components}

Native starches from different botanical sources vary widely in structure and composition (Hoover, 2001). Starch consists of two main structural components, amylose and amylopectin. Amylose is a relatively long, linear $\alpha$-glucan with few branches, containing approximately $99 \%$ $\alpha-(1,4)$ and up to $1 \%$ of $\alpha-(1,6)$ linkages, whereas amylopectin is a heavily branched structure, built from 
Fig 2.1 Schematic diagram of amylose (A) and amylopectin (B) (adapted from Tester and Karkalas, 2002). 

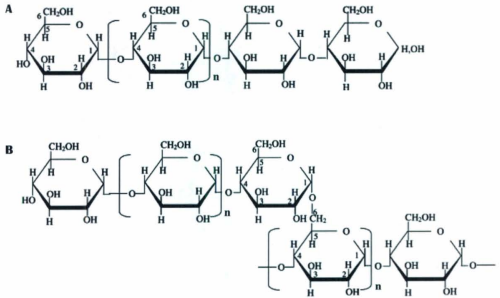
about $95 \% \alpha-(1,4)$ and $5 \% \alpha-(1,6)$ linkages (Fig 2.1) (Buléon et al., 1998; Pérez and Bertoft, 2010). Normal starches consist of $20-30 \%$ amylose and $70-80 \%$ amylopectin, but waxy (amylose-free) starches contain essentially 100\% amylopectin. High amylose starches (e.g., amylomaize V and VII and wrinkled pea) contain less than $30 \%$ amylopectin. The ratio of these two polysaccharides influences the functional properties of starch (Hoover, 2001). The proximate composition of starches of different botanical origin is presented in Table 2.3.

Amylose has a molecular weight range of approximately $10^{5}-10^{6} \mathrm{~g} / \mathrm{mol}$ (Buléon et al., 1998; Mua and Jackson, 1997), degrees of polymerization (dp) of 324-4920 monomer units and 9-20 branch points equivalent to 3-11 chains per molecule (Hizukuri, et al., 1981; Mua and Jackson, 1997; Takeda et al., 1987; Wang and White, 1994; Yashushi et al., 2002; Yoshimoto et al., 2000). Each chain contains approximately 200-700 glucose residues (Tester and Karkalas, 2002). Amylopectin is a much larger molecule than amylose with a molecular weight of $10^{7}-10^{9}$ (Biliaderis, 1998; Buléon et al., 1998; Morrison and Karkalas, 1990; Mua and Jackson, 1997). The dp of amylopectin is generally within the range 0.7 to $26.5 \times 10^{3}$ (Pérez and Bertoft, 2010).

A third component, intermediate materials, has been identified, especially in starches from plants with modified starch biosynthesis and in low amounts in normal starches (Banks and Greenwood, 1967, Klucinac and Thompson, 1998; Lansky et al., 1949; Wang et al., 1993; Yoon and Lim, 2003). High amylose maize genotypes (Buléon et al., 1998; Yuan et al., 1993) and wrinkled pea (Bertoft, 1993) are best known for their content of intermediate materials. 
Table 2.3 Proximate composition (\%) of starches of different botanical origin

\begin{tabular}{|c|c|c|c|c|c|}
\hline Starch source & Amylose & Lipid & Nitrogen & Phosphorus & Reference \\
\hline \multicolumn{6}{|l|}{ Potato } \\
\hline Normal & 28.1 & 0.20 & 0.09 & 0.1 & Guinaratne and Hoover (2002) \\
\hline Waxy & 0.0 & $\therefore$ & - & 0.066 & McPhetson and Jane (1999) \\
\hline High amylose & $59-72$ & $=$ & - & 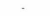 & Schwall et al. $(2000)$ \\
\hline Sweet potato & 22.6 & 0.14 & 0.02 & 0.014 & Petconi et al: (2006) \\
\hline Yam & 32.6 & 0.10 & 0.01 & 0.022 & Peroni et al: (2006) \\
\hline Cassava & 19.8 & 0.15 & 0.03 & 0.007 & Peroni et al (2006) \\
\hline Arrowroot & 20.8 & 0.17 & 0.02 & 0.018 & Peroai et al: (2006) \\
\hline Canna & 31.7 & 0.19 & 0.02 & 0.031 & Peroni et al, (2006) \\
\hline Ginger & 26.5 & 0.24 & 0.05 & 0.007 & Peroni et al. (2006) \\
\hline Wheat & 27.3 & 0.7 & 0,04 & - & Hoover and Vasanthan (1994a) \\
\hline Oat & 19.4 & 1.13 & 0.05 & - & Hoover and Vasanthan (1994a) \\
\hline \multicolumn{6}{|l|}{ Maize } \\
\hline Nermal & 29.9 & 0.75 & 0.02 & - & Hoover and Marnuel (1996) \\
\hline Waxy & 12 & 0.22 & 0,02 & . & Hoover and Manuel (1996) \\
\hline Amylomaize V & 65.5 & 0.95 & 0.03 & - & Hoover and Manuel (1996) \\
\hline \multicolumn{6}{|l|}{ Barley } \\
\hline Normal & $23.6-29.0$ & 0.91 & 0.3 & - & Lietal. (2001) \\
\hline Waxy & $0-6.44$ & 0.48 & 0.25 & - & Liefal (2001) \\
\hline High amylose & $41.7-44.5$ & 1.34 & 0.41 & - & Lietal. (2001) \\
\hline Lentil & 25.3 .32 .3 & $0.01-0.08$ & $0.03-0.09$ & - & Hoover et al. (2009) \\
\hline Simooth pea & $24-49$ & $0.02-0.08$ & $0.02-0.07$ & $\cdot$ & Hoover et al. $(2009)$ \\
\hline Wrinkled pea & $60.5-88.0$ & $0.8-0.84$ & $0.05-0.08$ & $*$ & Hoover et al. (2009) \\
\hline
\end{tabular}


These intermediate materials have structures and properties intermediate to those of amylose and amylopectin (Jane, 2009).

\subsubsection{Amylose}

\subsection{Fine structure of amylose}

Although all amylose molecules were once considered to be linear, many amylose molecules cannot be completely hydrolysed by $\beta$-amylase $[\beta$-amylase cannot hydrolyse or by pass $\alpha$ - $(1,6)$ linkages; its action stops at the branch points (see section 2.4.7.1)]. The incomplete $\beta$-amylolysis indicates that a certain degree of branching is present in some amylose molecules (Greenwood and Thompson, 1962; Hizukuri et al., 1981; Takeda et al., 1987). The $\beta$-amylolysis limit of amylose varies from $72-95 \%$ compared to $55-61 \%$ for amylopectin (Jane, 2009).

The amylose fraction of starch occurs in double-helical A- and B-amyloses and the single-helical $\mathrm{V}$-amylose. Both A and B forms feature left-handed belices with six glucose units per turn and seem to differ only in the packing of the starch helices (Buléon et al., 1998). The latter contains a channel-like central cavity that is able to include molecules such as iodine, dimethyl sulphoxide (DMSO), alcohols or fatty acids (Rappenecker and Zugenmaier 1998; Godet et al., 1995; Saitô et al., 1991; Imberty et al., 1991). In the single helical form, the interior surface of amylose is built up by $\mathrm{C}-\mathrm{H}$ groups and glycosidic oxygen atoms forming a lipophilic core, whereas all the polar hydroxyl groups are positioned on the outer surface of the helix (Hoover, 1998). The inner diameter of the amylose helix is controlled by the size of the complexing agent. Predominant in the $\mathrm{V}$ amylose family is the common form of $\mathrm{V}_{6}$ amylose, characterized by six glucose units per turn (Cardoso et al., 2007: Jouquand et al., 2006: Yamashita, et al., 
1971). However, for some guest molecules (e.g., iso- and tertiary butyl alcohols, benzoic acid, dimethyl sulphoxide, 1-napthol) the helix exhibits a different structure (e.g., $\mathrm{V}_{7}[7$ glucose residues per turn] and $\mathrm{V}_{8}$ [8 glucose residues per turn]) (Helbert et al., 1994; Shogren et al., 2006).

Formation of the double helix requires an alignment of two amylose molecules. Without the presence of complexing agents, the linear portion of amylose molecules gradually associate and form double helices. Kinetic studies have shown that the rate of double helix formation depends on the molecular size of the amylose, its concentration and the temperature (Jane, 2009). Gidley and Bulpin (1987) demonstrated with a model system of oligosaccharides that the minimum chain length required to form starch double helices is 10 . However, in the presence of longer chains, short chains $(\mathrm{dp}>6)$ can participate in the formation of double helices (Gidley and Bulpin, 1987).

\subsection{Conformation of amylose}

In a freshly prepared aqueous solution, amylose is present as a random coil (Hayashi et al., 1981). However, the random coil conformation is not stable. Amylose tends to form either single helical (inclusion) complexes with suitable complexing agents or to form double helices among themselves when no suitable complexing agent is available (French and Murphy, 1977; Jane 2009; Zobel, 1988a, 1988b). Both single and double helices result in lower energy states and are thermodynamically favourable (Jane, 2009). However, the local conformation of an amylose chain in an aqueous environment is a matter of dispute. Three different molecular models (Fig 2.2) (Banks and Greenwood, 1971, 1975; Cowie, 1961; Fujii et al., 1973; Kitamura 
and Kuge, 1989; Nguyen et al., 1976; Rao and Foster, 1963; Senior and Hamori, 1973; Yamamoto et al., 1982) have been proposed for amylose on the basis of data analysis of conformation-dependent properties in aqueous solvents and DMSO: 1) random coil with no helical nature (Fig 2.2a), 2) interrupted helix composed of helix regions (helical segment) with intervals of short regions of random coils between them (Fig 2.2b) and 3) deformed helix (loosely wound helical chain [Fig 2.2c]). Cheetham and Tao (1998b) have shown by ${ }^{13} \mathrm{C}$ NMR, optical rotation and viscosity measurements that amylose conformation in 100\% DMSO is helical rather than a random coil. With the addition of water, the intramolecular hydrogen bonding in amylose is gradually replaced by amylose-water intermolecular hydrogen bonds, leading to conformational changes. When the concentration of DMSO is decreased from $100 \%$ to $66.6 \%$, amylose conformation changes from tight helix to loose helix. A further decrease in DMSO concentration ( 66.6 to $33.3 \%$ ) causes a conformational change from loose helix to random coil (Cheetham and Tao, 1998).

\subsection{Location of amylose}

The exact location of amylose in starch granules still remains unresolved. Jane et al. (1992) showed, by cross-linking studies on potato and com starches using epichlorohydrin, that amylose appears to be interspersed among the amylopectin molecules rather than being located in bundles, bundles. Surface gelatinization studies (Jane and Shen, 1993) have shown that amylose is heterogeneously distributed within the granule. In potato and maize starches, amylose has been found to be concentrated mainly at the peripheral regions of the granules (Jane and Shen, 1993). However, Tatge et al. (1999) have shown, by studies of amylose synthesis in transgenic potato granules, that amylose is mainly present in the central region of the granule. 
Fig 2.2 Proposed models for amylose structure in aqueous solution (adapted from Banks and Greenwood, 1975; Yamamoto et al., 1982).
(a) random coil structure
(b) interrupted helical structure
(c) deformed helix structure 


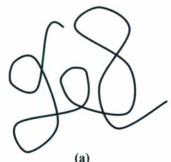

(a)

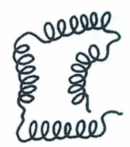

(b)

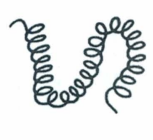

(c) 
Based on small angle X-ray scattering studies on barley, pea and maize starch granules, Jenkins and Donald (1995) postulated that in these starches amylose is mainly located in the amorphous growth rings. Atkin et al. (1999) have shown by enzyme-gold labeling and iodine staining studies on hydrated maize and potato granules that amylose is localized in distinct amorphous regions around the hilum. Distribution of amylose in starch granules of different botanical origin has been extensively investigated by many researchers (Blennow et al., 2003; Glaring et al, 2006; Wei et al, 2010) using APTS (8-amino-1,3,6-pyrenetrisufonic acid) coupled with confocal laser scanning microscopy (CLSM). These results also indicated that a high concentration of amylose is present in the granule center (region around the hilum).

Several studies have shown that in potato starch, amylose is co-crystallized (Fig 2.3) with the outer branches of amylopectin chains, forming double helices (Hoover and Vasanthan, 1994a; Jenkins and Donald, 1995; Saibene et al., 2008; Saibene and Seetharaman, 2010; Zobel, 1988a). However, the location of amylose relative to the amylopectin molecules in common corn starch is contradictory. Based on cross-linking studies, Jane et al. (1992) and Kasemusuwan and Jane (1994) suggested that amylose and amylopectin are closely associated in normal maize starch. Zobel (1988a) suggested that amylose may be partly co-crystallized with amylopectin chains in potato starch but they are separated in maize starch granules. Gerard et al. (2002) used mild acid hydrolysis to investigate the location of amylose with respect to the amorphous and/or crystalline regions in maize starch mutants. They observed a greater interaction of amylose and amylopectin in high-amylose maize starches than in common com starch. Co-crystallization of amylose and amylopectin has also been reported in wheat starches (Yuryev et al., 2007). . 
Fig 2.3 Proposed model for co-crystallization of amylose with amylopectin (Jenkins and Donald, 1995, Copyright Elsevier, reproduced with permission). 


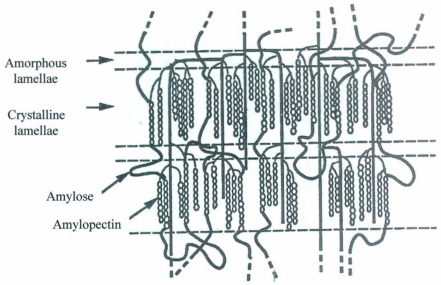




\subsection{Amylose iodine inclusion complex}

Amylose molecules are believed to be arranged in a left handed helix of an outer diameter of $13 \mathrm{~A}^{\circ}$, a $5.0 \mathrm{~A}^{\circ}$ central cavity, and a pitch of $8 \mathrm{~A}^{\circ}$, with each turn of the helix corresponding to six anhydroglucose units (Gidley and Bulpin, 1987; Rundle and French, 1943; Rundle, 1947). The cavity provides enough space to accommodate iodine atoms that are more or less linearly arranged with an internuclear distance of about $3.1 \mathrm{~A}^{\circ}$ (Fig 2.4a). This was first suggested by Hanes (1937) and later confimed by X-ray diffraction analysis by Rundle et al. (1943). During inclusion complex formation, an iodine molecule presumably enters into the cavity of the extended helix (Fig 2.4b), followed by its polarization by the oxygen atoms (Davis et al., 1994), which in turn helps the other iodine molecules to align within the cavity. The interaction of a polyiodide unit with the extended helix may form a tighter helix (Fig 2.4c), thereby providing greater stability to the polyiodine complex (Davis et al., 1994). Even though the amylose-iodine complex has been extensively studied, the mechanism of its formation remains controversial. Several researchers (Knutson et al., 1982; Saenger, 1984; Thoma and French, 1960; Yu et al., 1996) have shown that iodide ions (polyiodide units) are involved with the amylose-iodine complex. Calabrese and Khan (1999) suggested that the amylose-iodine complex can be explained by merely considering the presence of the $\mathrm{I}_{6}$ unit. However, there is ample evidence that in aqueous solution, the concentration of iodide ion is an important factor determining the extent to which iodine is bound by starch and that a significant fraction of bound iodine is iodide ion (Rendleman, 2003). Yu et al. (1996) have demonstrated that the primary structures of the polyiodide chains are composed of $\mathrm{I}_{3}{ }^{*}$ and $\mathrm{I}_{5}{ }^{*}$ subunits, which combine to form four dominant polyiodide chains such as $\mathrm{I}_{9}{ }^{3-}, \mathrm{I}_{11}{ }^{3 \cdot} \mathrm{I}_{13}{ }^{3 .}$ and $\mathrm{I}_{15}{ }^{3-}$. These polyiodide chains 
Fig 2.4 Proposed model for (a) amylose-iodine inclusion complex, (b) two extended helical segments of amylose joined by random non-helical structure and (c) two tighter helical segments of amylose joined by random non-helical structure (adapted from Calabrese and Khan,1999; Davis et al., 1994). 
(a)
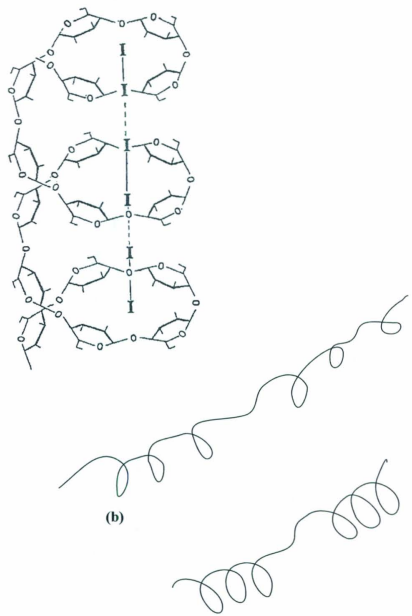

(c) 
give different absorbance spectra $(480-510,610-640,690-720$ and $730-760 \mathrm{~nm}$, respectively) when complexing with amylose. The average I----I distances of $3.1 \mathrm{~A}$ between these units are shorter than van der Waals radii. This facilitates electron delocalization along the polyiodide chain and explains the amylose-iodine spectrum (Saenger, 1984). However, the polyiodide units are not stable without the helix structure around (Davis and Khan, 1994).

The iodine binding capacity of pure amylose is reported to be $19-22 \%$ of its weight (Banks, et al., 1971), whereas for amylopectin it is about $1 \%$ (Gerard et al., 2001). Spectrophotometric and potentiometric methods are widely used for determining the amylose contents of starches. The color and wavelength of maximum absorbance of the amylose-iodine complex vary according to the degree of polymerization (dp) of the amylose helix: brown (dp 21-24), red (dp 25-29), redviolet (dp 30-38), blue-violet (39-46) and blue (dp>47) (John et al., 1983). This indicates that as the length of the helical segment in the unit of $d p$ increases, the number of iodine molecules which can be accommodated also increases. The increased iodine binding has been shown to result in a shift in the wavelength of maximum absorbance; $1 / \lambda_{\max }$ has been shown to be directly proportional to 1/dp up to a dp of about 100 (Banks et al., 1971; Bailey and Whelan, 1961). Yamamoto et al., (1982) have shown, by amylose-iodine interaction studies on potato amylose that a helical segment of amylose consists of fewer than 60 anhydroglucose residues. On this basis, they proposed that the interrupted helix (helical regions with intervals of random coils between them) is a good model for the amylose structure in aqueous solution.

Saibene and Seetharaman (2006) used iodine to study the architecture of the starch granule and reported the ability of granular corn starches to bind iodine at moisture contents as low as $8 \%$. 
Iodine binding in a starch granule requires a certain minimum level of mobility of the linear molecules, and that the water content plasticize the starch, thus increasing the ability to bind iodine by the formation of single helices. (Saibene and Seetharaman, 2006; Waduge et al., 2010). K/S spectra (the ratio of the absorption and scattering coefficients) and WAXS (wide angle X-ray scattering) have been used in these studies to investigate the intensity of the colour developed in granular starch when it is bound with iodine and to measure the effect of iodine binding to the crystallinity of the granules, respectively. The ability of iodine molecules to penetrate the starch granule likely depends also on the surface features of the granule, such as the presence of pores and channels (Wadugae et al., 2010).

\subsubsection{Amylopectin}

The structure of amylopectin is generally defined in terms of the cluster model (Robin et al, 1974; French, 1984) with polymodal chain length distribution (Figure 2.5) (Hizukuri, 1986) and a non-random nature of branching (Thompson, 2000). The cluster model for the amylopectin molecule describes the alternating amorphous and crystalline layers in starch (Robin et al., 1974).

\subsection{Fine structure of amylopectin}

Amylopectin unit chains are relatively short compared to amylose molecules and have a broad distribution file. The individual chains can be specifically classified in terms of their lengths and consequent position within starch granules (Hizukuri, 1985, 1986). In the classical nomenclature of Peat et al. (1952), A-chains are defined as unsubstituted by other chains and connected through an $\alpha-(1,6)$ linkage to the rest of the macromolecule, whereas B-chains are substituted by 
one or several other chains (A- and/or B-chains). In addition, each macromolecule contains a single C-chain that carries the sole reducing end group. Hanashiro et al. (2002) described the distribution of C-chains after labelling with a fluorescent dye. The chains covered the dp range 10-130, possessing a peak in size exclusion chromatograms at dp 40 for several amylopectin samples. B chains are classified as B1, B2, B3 or B4, depending on their length and the number of clusters they span (Hizukuri, 1986). B1 chains are short chains, being components of the unit clusters, whereas B2, B3 and B4 are long chains that act as connecting chains in the amylopectin molecule. Bertoft (1991) isolated groups of chains from maize amylopectin of intermediate length between the long chains and the major group of short chains. These were considered as subgroups of the originally defined $\mathrm{B} 1$ group and named $\mathrm{B} 1 \mathrm{~b}$ and $\mathrm{B} 1 \mathrm{c}$, whereas the shorter, major group was B1a. The chain lengths of A, B1, B2, B3 and B4 chains of amylopectin from different starch sources have been shown to be in the range of 12-16, 20-24, 42-48, 69-75 and 101-119, respectively (Hizukuri, 1986; Wang and White, 1994). The ratio of A- to B-chains depends on the starch source and is typically of the order of $<1.1$ to $>2.1$ on a molar basis, or $<0.5: 1$ to $>1: 1$ on a weight basis (Morrison and Karkalas, 1990; Tester and Karkalas, 2002). The ratio of the long to short branch chains affects the shape of AMP (e.g., cylindrical versus conical shapes), which influence their packing and, in turn, the morphology and size of the starch granule (Jane, 2007). The average chain length ( $\overline{\mathrm{CL}})$ of amylopectin is typically $18-25$ units (glucose residues) (Hizukuri, 1985, 1986) and depends on the type of crystallinity of the granular starch (see section 2.3.3.2). A-type crystalline starches typically possess shorter chain length than B-type crystalline starches (Hizukuri, 1985). Pérez and Bertoft (2010) suggested that the $\overline{\mathrm{CL}}$ value gives only a poor description of the true structure of amylopectin, because both A- and Btype crystalline starches possess short and longer chains. 
Fig 2.5 A cluster model of amylopectin proposed by Hizukuri (1986). A and B denote nomenclature of branch chains, $\Phi=$ reducing end, c.l. = chain length as degree of polymerization (Hizukuri, 1986, Copyright Elsevier, reproduced with permission). 


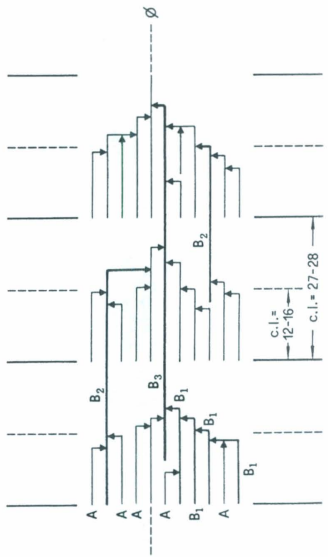




\subsubsection{Amylopectin-iodine complex}

Blue colour is characteristic of the complex of iodine with amylose, whereas a reddish violet colour is characteristic of the amylopectin-iodine complex. This fact is explained by the different structures of the above complexes. Amylopectin-iodine complex formation primarily involves A-chains (Davis and Khan, 1994). Davis and Khan (1994) found that for four iodine atoms, there were 11 cyclic glycoside fragments in the formation of a chromophore group in the amylopectin-iodine complex, whereas this ratio varied from 6 to 17 in the amylose-iodine complex. However, studies showed that the linear A-chains have an average chain length of 12 16 anhydroglucose units (see sec. 2.3.1.1). Davis and Khan (1994) postulated that longer Achains in amylopectin may not be available for iodine binding if pairs of them are involved in double helix formation.

\subsubsection{Minor components}

\subsubsection{Phosphorus}

Phosphorus is one of the non-carbohydrate constituents present in starches which significantly affects their functional properties (Karim et al., 2007; Lim et al., 1994; Lin and Czuchajowska, 1998; McPherson and Jane, 1999). Phosphorus is present as phosphate monoesters and phospholipids in various starches. The phosphate monoesters are covalently bound to the amylopectin fraction of the starch (Blennow et al., 2000; Schoch and Maywald, 1956; Schoch, 1942). Inorganic phosphate is present in some starches (Kasemsuwan and Jane, 1996; Lim et al., 1994). Tuber starches such as potato have been shown to have a higher phosphate monoester content (upto 0.09\%) than cereal starches (Blennow et al., 1998; Hizukuri et al., 1970; Lim et 
al., 1994; Kasemsuwan and Jane, 1996; Mcpherson and Jane, 1999). Takeda and Hizukuri (1982) have shown, in studies on potato, that amylopectin contains one phosphate monoester group per 317 glucose residues, equivalent to one phosphate group per 13 branch chains.

The phosphate groups are located mainly on C-6 and C-3 of the glucose units of amylopectin (Hizukuri et al., 1970). The enzyme $\alpha$-glucan water dikinase (GWD) is responsible for the phosphorylation at the C-3 and C-6 positions. Approximately $60-70 \%$ of the phosphate groups are bound as monoesters to the C- 6 position of the glucosyl units, whereas $30-40 \%$ are monoesterified to the C-3 position (Tabata and Hizukuri, 1971; Bay-Smidt et al., 1994). A small fraction (1\%) may be linked to the C-2 position (Hizukuri et al,, 1970; Jane et al., 1996). It is believed that amylose is not an efficient acceptor of phosphate groups (Kotting et al., 2005; Lorberth et al., 1998; Ritte et al., 2006; Ritte et al., 2002). Longer chains (dp 30-100) of amylopectin act as better substrates for GWD (Ritte et al., 2002; Mikkelsen et al., 2004) and the degree of phosphorylation is correlated to the chain length of amylopectin (Blennow et al., 1998). A unit chain may contain one or more phosphate groups (Blennow et al., 1998) but no phosphate groups are located on the non-reducing end (Takeda and Hizukuri, 1981) or closer than nine glucosyl units from an $\alpha-(1,6)$ branch point (Takeda and Hizukuri, 1982). On a molar basis, approximately $50 \%$ of the phosphorylated chains belong to the category of long B-chains $(C L>35)$ and the rest are $\mathrm{B}_{1}$-chains, whereas A-chains apparently are non-phosphorylated (Noda et al., 2005). Surface gelatinization studies have shown that phosphate monoesters are more concentrated at the core (hilum) than in the periphery of the potato starch granule (Jane and Shen, 1993). This is explained by the fact that amylopectin, at the core, has longer B-chains (B2 and B3) than that at the periphery (Pan and Jane, 2000). Furthermore, studies showed that small 
granules have greater phosphate content than larger granules (Jane and Shen, 1993; Noda et al., 2005).

The phosphorus content in potato starch has been reported to be influenced by growing conditions, temperature and storage (Smith, 1987). In potato starch, the high phosphate monoester content contributes to high viscosity, high transparency, water binding capacity and freeze thaw stability (Craig et al., 1989; Swinkels, 1985). This has been attributed to repulsion between negatively charged phosphate groups on adjacent amylopectin chains that hinders strong interactions between double helices (Hoover, 2010). Furthermore, studies showed that the phosphate monoester content of potato starch is inversely proportional to the crystallinity (Muhrbeck et al, 1991) and the gelatinization enthalpy (Muhrbeck and Eliasson, 1991) of the starch. This indicates that the phosphate monoesters are present within the crystalline region of the starch granule (Jane 2009).

\subsubsection{Lipid}

The lipid content and composition of starch granules vary from plant to plant (Morisson, 1988). Lipids associated with cereal starch granules have been found to occur on the surface as well as inside the granules (Morrison 1981). Surface lipids are occasionally referred to as non-starch lipids, which consist of triacylglycerols, polar lipids (glycolipids and phospholipids) and small amounts of diglycerides and free fatty acids (Morrison 1981, Galliard and Bowler 1987). Lipids present on the surface of cereal starches, particularly in the case of small granules $(10 \mu \mathrm{m})$, are able to encapsulate the granules with a thin lipid film (Galliard and Bowler, 1987). Lee and BeMiller (2008) postulated that what has been reported to be non-starch lipid of starches 
containing channels (maize, sorghum, millet, wheat, rye, barley) is the lipid lining the channels, i.e., lipid at least primarily associated with internal surfaces rather than the external surface of granules. Internal lipids are mainly monoacyl lipids (lysophospholipids) and free fatty acids (Acker, 1977; Morrison, 1981). These two groups of lipids (surface and internal lipids) differ in extractability from granules with common lipid solvents. Surface lipids are extractable with cold solvents (chloroform and ether), whereas internal lipids can be extracted with hot aqueous alcohol (propanol-water 3:1 [v/v]) (Vasanthan and Hoover, 1992). Surface and internal lipids have been shown to be present in the free state as well as bound to starch components, either in the form of amylose inclusion complexes or linked via ionic or hydrogen bonding to hydroxyl groups of the starch components (Morrison, 1988; Morrison, 1995; Vasanthan and Hoover, 1992). The major fatty acids in neutral lipid fractions are palmitic acid (wheat, corn, potato and cassava) and linoleic acid (rice and lentil). The glycolipid fractions of cereal and cassava starches have been reported to contain linoleic acid and palmitic acid, respectively. However, in phospholipid fractions, all starches contain palmitic acid as major fatty acid (Vasanthan and Hoover, 1992).

Generally, cereal starches (e.g., com, wheat, rice) contain relatively high levels of lipid (0.2$0.8 \%$ ) whereas, tuber (e.g., potato) and root (e.g., tapioca) starches have lower levels of lipids (0.1-0.2\%). The amount of lipid-complexed amylose ranges from less than 15 to more than $55 \%$ of the amylose fraction in cereal starches (Morrison, 1995). In most cereal starches, there is a strong correlation between the amylose and lipid contents. Most waxy starches have comparatively low amount of lipids (Morrison, 1984; South et al., 1991). 


\subsection{Amylose-lipid inclusion complex}

As discussed earlier (sec 2.3.1.1.1), amylose is known for its ability to form inclusion complexes with hydrophobic ligands (guest molecules). Lipids and linear alcohols form complexes with six glucose residues per turn with an inner diameter of $0.48 \mathrm{~nm}$. Consecutive turns of helices are stabilised by numerous intra- and interhelical van der Waals bonds and hydrogen bonds (Biliaderis and Galloway, 1989; Rappenecker and Zugenmaier, 1981). In the amylose-lipid inclusion complex, the aliphatic part (hydrocarbon chain) of the lipid is located inside the lipophilic core of the amylose helix, whereas the polar group lies outside of the helix (Fig 2.6) (Carlson et al., 1979).

DSC thermograms of normal cereal starches (e.g, maize and wheat) show an additional thermal transition peak at a higher temperature than the starch gelatinization peak (Donavon and Mapes, 1980; Kugimiya et al., 1980). This peak has been attributed to the melting of the amylose-lipid complex (Biliaderis et al., 1985; Bulpin and Welsh, 1982; Donavon and Mapes, 1980; Eliasson and Krog, 1985; Hoover and Hadziyev, 1981; Kugimiya and Donavan, 1981). NMR ( ${ }^{13} \mathrm{C}-$ crosspolarization/magic angle spinning $\left[{ }^{13} \mathrm{C}\right.$-CP-MAS] NMR spectroscopy) studies have provided evidence for the existence of amylose-lipid complexes in native barley, maize, rice and oat

starches (Morrison et al., 1993a, 1993b). ${ }^{13} \mathrm{C}$-CP-MAS NMR produces a broad resonance with a chemical shift of $31.2 \mathrm{ppm}$, a characteristic of mid-chain methylene carbons of fatty acids in the V-amylose complex (Fig 2.7). The results showed that up to $43 \%$ of the amylose in non-waxy rice starch, $33 \%$ in oat starch and $22 \%$ in normal maize and wheat starch granules are complexed with lipids. Based on this observation, Morrison et al. (1993a) postulated that amylose in cereal starches occurs as two fractions, lipid amylose and free amylose. 
Fig. 2.6 Schematic illustration of amylose-lipid complex (adopted from Carlson et al., 1979). 


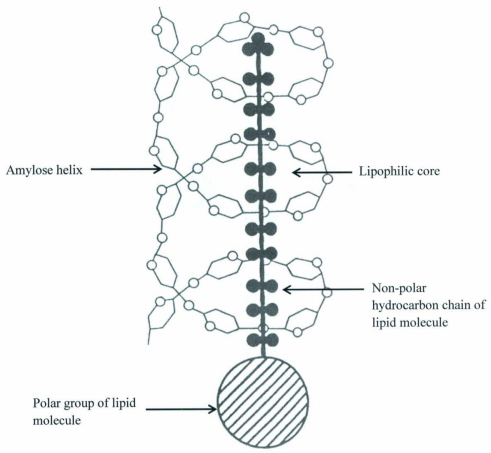


Fig $2.7{ }^{13} \mathrm{C}$-CP-MAS NMR spectra of V-amylose in waxy barley starch (Chalkey Glen), with inset of the $10-50 \mathrm{ppm}$ region at $\times 20$ scale expansion. The marked resonance is for mid chain methylene carbons of fatty acids in lysophospholipid with a chemical shift of 31.2 ppm (A). Resonance from polysaccharide carbons (B-E) are $\mathrm{B}=\mathrm{C}-6(61.3 \mathrm{ppm}), \mathrm{C}=$ unresolved C-2, C-3, C-5 (71.4 ppm), D = C-4 (80.9 ppm), E = C-1 (100.5 ppm) with some helical amylose (103 ppm) (Morrison et al., 1993b, Copyright Cereal Chemistry, reproduced with permission). 


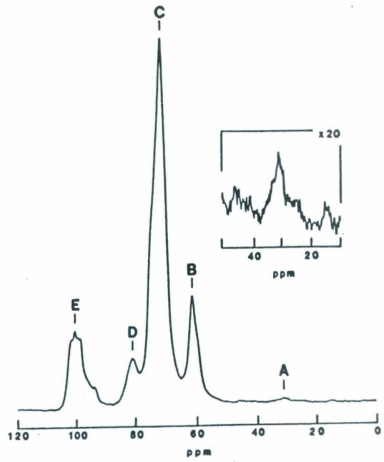


The amylose-lipid complex in the solid state shows the existence of two structurally different forms: type I (form I) and type II (form II) based on the crystallization conditions (temperature, type of ligand). Type I gives an amorphous X-ray pattern, whereas type II exhibits the typical Vtype pattern (Biliaderis and Galloway, 1989; Biliaderis and Seneviratne, 1990). Type I (amorphous complex) is found in most native cereal starches, which display a melting temperature at $94-100^{\circ} \mathrm{C}$. The melting temperature of the amylose-lipid complexes increases with the chain length of the fatty acid. Type II lamellar crystalline forms melt at $100-125^{\circ} \mathrm{C}$ (Fig 2.8) (Karkalas et al., 1995; Raphaelides and Karkalas, 1988). Therefore, the V-type X-ray pattern has not been observed in native starches, except for high $(>30 \%)$ amylose starches such as wrinkled pea, amylomaize and some other maize genotypes (sugary [su], dull [du]) (Galliard and Bowler, 1987; Gernat et al., 1993). The absence of a V-type pattern in native starches does not necessarily indicate the absence of amylose-lipid complexes. It merely proves that amyloselipid complexes are not arranged in crystalline domains that can be detected by $\mathrm{X}$-ray diffraction techniques (Hoover, 1998).

Formation of V-type amylose has been observed during swelling and gelatinization of starch granules (Biliaderis et al., 1986b; Morisson, 1988), on heat-moisture treatment (Hoover et al., 1996; Lim et al., 2001), on extrusion cooking of lipid-containing cereal starches (Mercier et al., 1980), and after addition of monoacyl lipids to starch under appropriate conditions (Hoover Hadziyev, 1981). The amylose-lipid inclusion complex has been shown to influence the gelatinization temperature, granular swelling and solubility, amylose leaching, paste viscosity, retrogradation, and enzymatic hydrolysis (Debet and Gidley, 2006; Guraya et al., 1997; Holm et al., 1983; Hoover, 2010, 1998; Tester and Morrison 1990; Vasanthan and Hoover, 1992). These 
Fig. 2.8 A typical DSC thermal curve (50\% solids) of a cereal starch showing the different melting transitions and the corresponding structural domains undergoing a phase change. $\mathrm{M}_{1}$ and $\mathrm{M}_{2}$ : melting of amylopectin crystallites at intermediate moisture content; $\mathrm{M}_{3}$ : melting of form 1 amylose-lipid complex; $\mathrm{M}_{4}$ : melting of form II amylose-lipid complex (adapted from Billiaderis, 1998; Biliaderis and Galloway, 1989; Biliaderis and Seneviratne, 1990). 


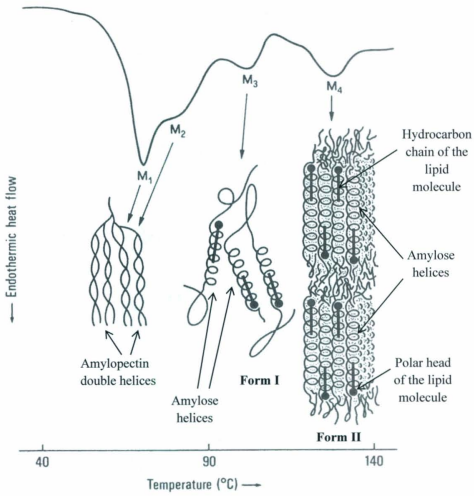


inclusion complexes are effective in preventing the stickiness of starch (Hoover and Hadziyev, 1981), improving structural integrity in cereal kernels (parboiled rice) during cooking (Biliaderis et al., 1993), increasing the free-thaw stability and an anti-staling effect in bread and biscuits (Riisom et al., 1984), and improving the texture in products containing extruded starch (Mercier et al., 1980).

\subsubsection{Proteins}

The nitrogen content of starch is a direct indication of crude protein $[\mathrm{N} \times 6.25]$ content. Generally, a starch granule comprises a number of nitrogen fractions such as protein, peptides, free amino acids, amides, enzymes and nucleic acids (Eppendorfer, 1979; Lineback and Rasper, 1988; Swinkels, 1985). Among these nitrogen fractions, pure protein represents on average about $50 \%$ of the nitrogen of potato tubers, and may range from $34 \%$ to $70 \%$ (Shewry, 2003). Maize and waxy maize starches contain a considerable amount of protein $(0.25-0.8 \%)$ compared to tuber and root starches (potato, $0.06 \%$, and tapioca, 0.01\%) (Swinkels, 1985; Buléon et al., 1998). The protein content of purified starch is a good indicator of starch purity.

Proteins are known to be associated with both the surface and the interior of starch granules (Baldwin, 2001). Rahman et al. (1995) suggested that the intrinsic proteins are almost entirely enzymes involved in starch synthesis. Approximately $10 \%$ of the starch proteins appeared to be associated with the granule surface (Galliard and Bowler, 1987). Surface proteins in starches are often dominated by storage proteins, particularly endosperm storage proteins in cereals (Schofield and Greenwell, 1987; Mu-Forster and Wasserman, 1998). The presence of starch granule surface proteins have been reported for potato and tapioca, (Yoshino et al., 2005), wheat 
(Greenwell and Schofield, 1986; Seguchi and Yamada, 1989), maize (Imam, 1989), rice (Villareal et al., 1986), barley (Prentice and Stark, 1992) and mung bean (Oates, 1990) starches. Surface associated proteins of starch granules can be readily extracted with dilute salt (sodium dodecyl sulphate) under mild conditions (Greenwell and Schofield, 1986; Seguchi and Yamada, 1989) that cause no disruption of granules, whereas the internal proteins cannot be extracted without disruption of the granule crystalline structure via chemical or thermal gelatinization (Debet and Gidley, 2006; Gillian et al., 1981; Mu-Forster et al., 1996).

\subsubsection{Supramolecular structure}

Starch granules consist of concentric alternating amorphous and semicrystalline growth rings (Fig 2.9). They grow by apposition from the hilum of the granule. The number and thickness of these layers depend on the botanical origin of the starch. They are typically $120-400 \mathrm{~nm}$ in thickness (French, 1984) and are considered to represent diurnal fluctuations in the deposition of starch in storage tissues (Donald et al., 2001; Gallant et al., 1997; Sevenou et al., 2002). The low-density amorphous rings consist of amylose and amylopectin in a disordered conformation, whereas the dense semicrystalline rings are formed by a lamellar structure of alternating crystalline and amorphous regions with a repeat distance of 9-11 nm (Cameron and Donald, 1992; Donald, 2004; Donald et al., 2001; Yuryev et al., 2004). The crystalline regions of the lamellae are formed mainly by double helices of amylopectin side chains packed laterally into a crystalline lattice, whereas amorphous regions contain amylose and the amylopectin branching points. The longer amylopectin chains are considered to pass from the crystalline region into the amorphous region of the lamellae (Qi et al., 2004). 
Fig 2.9 Schematic representation of the starch granule: (a) amorphous and semi-crystalline growth rings in a starch granule, (b) amorphous and crystalline lamellae in a stack and part of an amorphous growth ring, (c) aligned double helices (from amylopectin side chains) within a crystalline lamella and amylopectin branch points within an amorphous lamella (d) blocklet (adapted from Donald et al., 1997; Myers et al., 2000). 
Semicrystalline growth ring

(a)

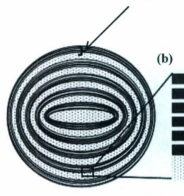

Amorphous lamella

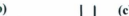

(c)

Cluster

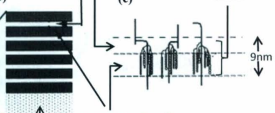

$\uparrow \quad \begin{gathered}\text { Crystalline lamella } \\ \uparrow\end{gathered}$

Amorphous growth ring

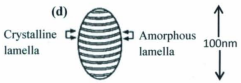


Based on scanning electron microscopy observations, Gallant et al. (1997) suggested that both semi-crystalline and amorphous growth rings are subdivided into large $(20-500 \mathrm{~nm}$ in diameter) and small (25 $\mathrm{nm}$ in diameter) spherical blocklets, respectively. On average, two end-to-end blocklets would constitute a single semicrystalline growth ring. These blocklets have an average size of $100 \mathrm{~nm}$ in diameter and are proposed to contain 280 amylopectin side chain clusters. In this view, one blocklet in the semi-crystalline growth ring contains several amorphous and crystalline lamellae (Fig. 2.9d) (Le Corre et al., 2010; Vandeputte and Delcour, 2004). Generally, B-type starches (e.g., potato) contain larger (400-500 nm) blocklets than A-type starches (e.g. wheat starch, $25-100 \mathrm{~nm}$ in diameter). In potato starch, the granule surface (approximately the outer $10 \mu \mathrm{m}$ ) is predominated by large blocklets $(400-500 \mathrm{~nm}$ ), whereas the granule centre is dominated by small blocklets (Perez and Bertoft, 2010).

Amylopectin clusters may contain amylose tie-chains, which are amylose molecules that pass through both the crystalline and amorphous layers (Jane et al., 2007; Qi et al., 2003). It has been suggested that these amylose tie molecules are in a straightened conformation in crystalline regions and in a disordered conformation in amorphous regions (Fig 2.10a) (Kozlov et al., 2007; Matveev et al., 1998; Yuryev, 2007). Jenkins and Donalds (1995) concluded that an increase in amylose content has the effect of increasing the size of the crystalline lamella and acts to disrupt their packing (Fig 2.10b). Two mechanisms to explain this disruption have been introduced: first, co-crystallization between amylose and amylopectin chains and, second, the penetration of amylose into amorphous regions. However, in high-amylose starches, amylose helices may contribute to the crystallinity of granules (Buleon et al., 1998; Hoover, 2001; Matveev et al., 2001; Tester et al., 2004). 
Fig 2.10 Localization of amylose chains in amylopectin clusters. (a) Yeryev's model for amylose chains localization in amylopectin cluster (adapted from Yeryev, 2007); (b) A proposed mechanism to explain the disruption of amylopectin double helical packing by amylose; (A) Amylopectin structure with no amylose present - small crystalline lamellar size; (B) Cocrystallinity between amylose and amylopectin moves a number of the amylopectin chains out of register - increased crystalline lamellar size (Jenkins and Donald, 1995, Copyright Elsevier, reproduced with permission). 
a)

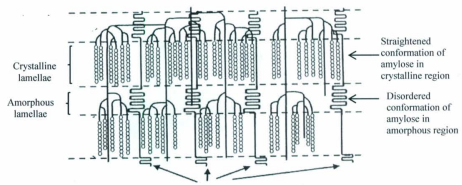

Amylose tie chains (amylose

molecules that pass through both the

crystalline and amorphous region)

b)

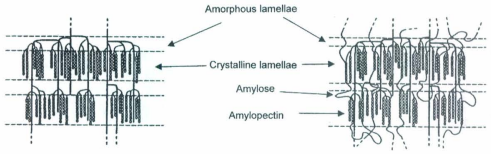

A 


\subsubsection{Birefringence}

The wave model of light describes light waves vibrating at right angles to the direction of propagation with all vibration directions being equally probable. This is referred to as "common" or "non-polarized" white light. In contrast, polarized light waves vibrate in just a single plane (McMohan, 2004). A polarizing filter can convert unpolarized light into polarized light. If two polarizing filters are crossed (oriented at right angles to one another), no light will pass through the second filter. Certain biological materials may act like polarizing filters. The biological material must have a highly ordered internal structure, approaching a crystalline pattern of organization, to partially polarize light. If such materials are placed between crossed filters, some of the light waves are polarized in a new direction and will pass through the second filter. This property is known as birefringence because the material bends or refracts light into two planes. The biological material will be bright against a dark background since the crossed polarizing filters still prevent light from passing through everywhere else (McMahon, 2004).

When starch granules are viewed under cross polarized light, they show birefringence, producing what is often described as the Maltese cross pattern. Birefringence is believed to be due to the radial orientation of the external chains of the amylopectin crystallites in the granule (Evans and Thompson, 2004). According to birefringence maps (French, 1972), the amylopectin chains are symmetrically and radially oriented (Fig 2.11). The Maltese cross pattern is the complementary dark pattern that divides the four light quadrants. The dark regions result when the optic axes in that part of the granule align with one of the polarizing filters (Evans et al., 2003). 
Fig 2.11 Schematic model of the organization of a starch granule (Jane, 2009, Copyright Elsevier, reproduced with permission). 


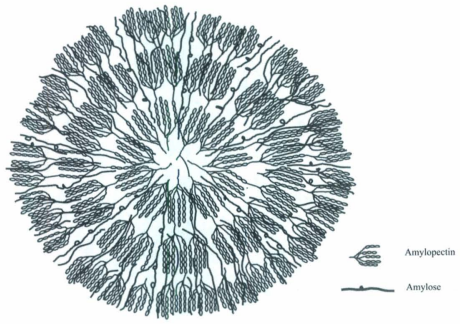




\subsubsection{Starch crystallinity}

Starch crystallinity is attributed to structural elements of amylopectin (Jenkins and Donald, 1995). The unbranched outer A-chains and the shortest inner branched chains (B1) of amylopectin can form double helices, which can crystallize into different crystal types. This crystallization or double helical formation can occur either in the same amylopectin branch cluster or between adjacent clusters in three dimensions and is called the superhelical structure Gallant et al, 1997). Double helical content in starch has been extensively researched by ${ }^{13} \mathrm{C}$ cross polarization magnetic angle spinning nuclear magnetic resonance spectroscopy $\left({ }^{13} \mathrm{C}\right.$ CPMAS-NMR) (Bogracheva et al., 2001; Lopez-Rubio et al., 2008; Paris et al., 1999; Tamaki et al., 1998; Yusuph et al., 2003). These studies showed that crystallinity of starch increases with increasing double helical content (Table 2.4). Based on this observation, Lopez-Rubio et al. (2008) suggested that most of the double helices present in starch granules are packed into crystalline structures.

Wide angle X-ray scattering (WAXS) is widely used to study the structure of semi-crystalline carbohydrate polymers and it provides information of long range order such as the packing of double helices into ordered arrays (Sevenou et al., 2002). Starch granules, depending on their botanical origin and composition (amylose/amylopectin ratio, amylopectin branch length), exhibit two types of WAXS patterns, which are associated with two crystalline polymorphic forms: A-type and B-type. A-type (Fig 2.12A) is mainly found in normal cereal starches and the B-type (Fig 2.12B) is observed in tubers and high amylose starches. 
Table 2.4 Double helical content and crystallinity of starches

\begin{tabular}{lcc}
\hline Starch & \% Double helix (NMR) & \% Crystallinity \\
\hline Waxy maize & 47 & 46 \\
Rice & 38 & 37 \\
Potato & 44 & 45 \\
Barley & 21 & 22 \\
Penford wheat & 22 & 23 \\
NB1 wheat & 29 & 25 \\
Gelose 80 & 26 & 22 \\
Hylon VII & 24 & 21 \\
\hline
\end{tabular}

(adapted from Lopez-Rubio et al., 2008a) 
Fig 2.12 X-ray diffraction patterns of A-, B-, C-type starches with their characteristic d-spacings (adapted from Zobel, 1988b). A-type; B-type; C-type 


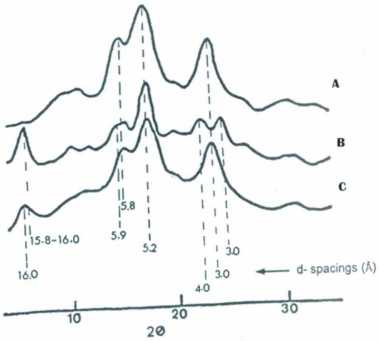


Legume, root and some fruit and stem starches contain significant fractions of both crystalline types, resulting in a combined WAXS pattern referred to as C-type (Fig 2.12C) (Buleon et al., 1998; Zobel, 1988; Cairns et al., 1997; Hizukuri et al., 1985; Imberty et al., 1991; Lineback, 1984; McPherson and Jane, 1999; Sevenou et al., 2002). Another crystal polymorph is often observed in granular starches, the so-called V-type (Fig 2.13) which, in contrast to the double helical nature of the A and B crystal structures, has been described to arise from single amylose helices (Lopez-Rubio et al., 2008a; Saibene et al., 2008), some of which are complexed with endogenous granular lipids (Morrison et al., 1993).

The double helices within the A- and B-type polymorphic forms are essentially identical with respect to helical structure (Gidley and Bulpin, 1987; Imberty et al., 1991). Within the double helix, interstrand stabilization occurs through $0-2 \cdots 0-6 \mathrm{H}$-bonding between the two chains as well as through numerous van der Waals interactions. The inner diameter of the double helix cavity is $\sim 0.35 \mathrm{~nm}$ (i.e., it does not allow any water molecules to pack inside the helix), whereas the outer diameter is $\sim 1.03 \mathrm{~nm}$ (Biliaderis, 1998). However, the A- and B-type polymorphs, differ in the packing arrangement of the double helices and in the water content. Wu and Sarko (1978a, b) postulated that both A- and B-polymorphs are right-handed, parallel-stranded double helices packed in anti-parallel fashion (Fig 2.14). Later, an improved structure of A- and Bpolymorphs was proposed by Imberty et al. $(1998,1999)$. This new model was still based on parallel-stranded double helices, but they were left-handed instead of the previous right-handed ones. In addition, these double helices were packed in a parallel fashion as opposed to Sarko's structure, where the packing was antiparallel. 
Fig 2.13 V-type X-ray diffraction pattern of amylose-lipid complex showing characteristic dspacings (4.4-4.3, 6.5-6.8, 11.3-12) (adapted from Zobel, 1988b). 


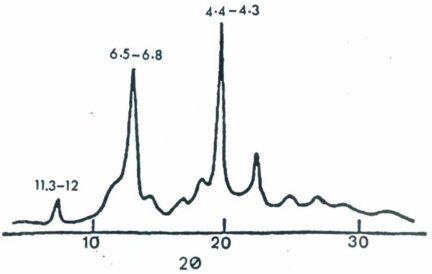


Fig 2.14 Arrangement of double helices of A-type and B-type crystallites in starch granules (adapted from Wu and Sarko, 1978a; 1978b). 


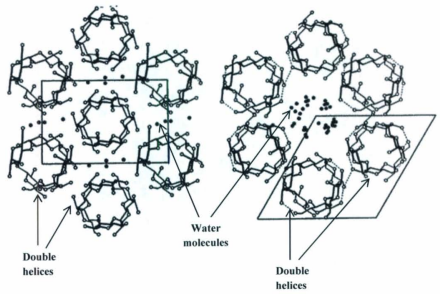


For the A-polymorph, chains are crystallized in a monoclinic lattice (unit cell parameters $a=$ 21.24 $, b=11.72 \AA, c=10.69 \AA$ and $\alpha=\beta=90^{\circ}, \gamma=123.5$ ) having maltotriose as the repeating (asymmetric) unit and four water molecules per unit cell. The B-polymorph crystallizes in a hexagonal unit cell $\left(a=b=18.5 \AA, c=10.4 \AA\right.$ and $\left.\alpha=\beta=90^{\circ}, \gamma=120\right)$ with a more open hexagonal packing and 36 water molecules per unit cell (Fig 2.15) (Imberty and Perez, 1988, Imberty et al., 1988). The water molecules fill the larger central channel formed by the hexagonically packed double helices; half of the water is tightly bound to the chains, and the other half is connected only to other water molecules (Biliaderis, 1998). The C-polymorphic structure is a mixture of A and B unit cells (the proportions vary among pulse starches) and, therefore, is considered to be intermediate between the A and B forms in packing density and structure (Buleon et al., 1998; Caims et al., 1997; Hizukuri et al., 1980; Imberty et al., 1991; Lineback, 1984; McPherson and Jane, 1999; Sevenou et al., 2002).

Hizukuri et al. (1983) postulated that amylopectin chain length was a determining factor for crystalline polymorphism. The relatively short exterior chains of cereal starch amylopectin molecules (chain length 14-20, or $<20$ ) favour the formation of A-type crystalline polymorphs (of double helices), with the longer exterior chains of tuber starches (chain length 16-22, or $>22$ ) favouring the formation of B-type polymorphs (Hizukuri et al., 1986). Jane et al. (1997) showed that the B-type amylopectin branch points are clustered, forming a smaller amorphous lamella, whereas A-type amylopectin branch points are scattered in both the amorphous and the crystalline regions, giving more flexibility to double helices to pack closely (Fig 2.16). 
Fig 2.15 Structure of A- and B-type unit cells.

A] Structure of A- starch. Chains are crystallized in a monoclinic lattice. In the unit cell, 12 glucopyranosyl units are located in two left handed, parallel-stranded double helices, packed in a parallel fashion. For each unit cell, four water molecules are located between the helices. (Imperty et al., 1988, Copyright Elsvier, reproduced with permission).

B] Structure of B-starch. Chains are crystallized in a hexagonal lattice, where they pack as an array of left-handed, parallel-stranded double helices, packed in a parallel fashion. Thirty six water molecules represent $27 \%$ hydration. Hydrogen bonds are indicated as broken lines (Imperty and Perez, 1988, Copyright John Wiley and Sons, reproduced with permission).

Projection of the structure onto the $(\mathrm{a}, \mathrm{b})$ plane. Hydrogen bonds are indicated as broken lines. Water molecules are indicated by closed circles. 


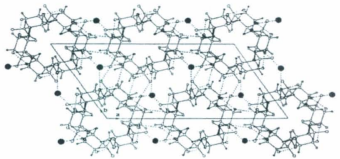

A

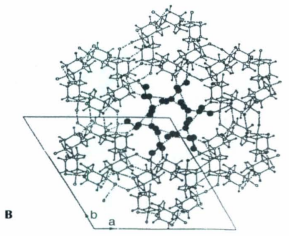


Fig 2.16 Proposed models for A-type (waxy maize starch) and B-type (potato starch) amylopectin branching patterns (Jane et al., 1997, Copyright Elsevier, reproduced with permission). 
A-type starch

(waxy maize starch)

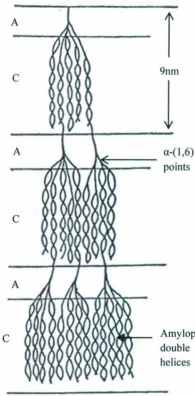

B-type starch

(potato starch)

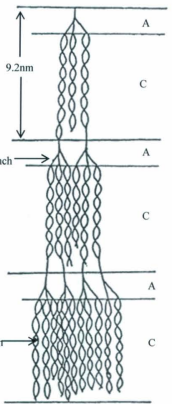
A: amorphous
C: crystalline 
Certain pea mutants show different proportions of A- and B-type polymorphs within the starch granule and are used as a model to understand the development of the different polymorphs (Hedley et al., 2002). Microfocus synchrotron WAXS mapping has confirmed that the Bpolymorphs occur in the core of smooth pea starches, whereas the A-polymorph is located at the periphery (Buleon et al., 1998). Wang et al. (2007a, 2007b) showed by acid hydrolysis that the core part of C-type starch (Chinese yam) was preferentially hydrolyzed and that hydrolyzed starch showed an A-type diffraction pattern, suggesting that B- and A-type are present mainly in the amorphous and crystalline domains respectively. It is now recognized that many regular cereal starches thought to contain only the A-polymorph actually contain small amounts of the B-polymorph (Vermeylen et al., 2004). Ziegler et al. (2005) postulated that the hilum region in most granules contains B-type crystallites, and that the overall WAXS pattern is determined by the respective polymorphs contained in the core and periphery, the weight fraction of each region and the ratio of their crystallinity.

\subsection{Degree of crystallinity}

The degree of crystallinity can be defined as the percentage of the crystalline regions with respect to the total material (Lopez-Rubio et al., 2008a). The amount of crystallinity within starch granules has been reported by many authors. Generally, degree of crystallinity of native starch granules is in the range of $15-45 \%$ (Lopez-Rubio et at., 2008a; Zobel, 1988b) depending on the botanical origin, amylose/amylopectin ratio, moisture content and methods used for the crystallinity calculation. The sharpness of the X-ray diffraction pattern of starch granules depends on their water content, B type being more sensitive to hydration than A-type starch (Cleven et al., 1978; Buléon et al., 1982). Studies have shown that there is an optimum moisture 
content (about $27 \%$ ) at which maximum crystallinity is observed, whereas a minimum of $8 \%$ water is necessary for the starch to exhibit a X-ray diffraction pattern (Imberty and Perez, 1988). Starch crystallinity was initially calculated by Sterling (1960) and Nara (1978) following the methodologies developed by Hermans (1948) and Wakelin (1959) for cellulose based on the two phase concept, which assumes that relatively perfect crystalline domains (crystallites) are interspersed with amorphous regions. This estimates the degree of crystallinity by separating the diffraction pattern into sharp (crystalline) and diffuse (amorphous) components. Most researchers used this method with slight modifications to calculate starch crystallinity. However, this concept does not fit with the improved understanding of starch structure that encompasses intermediate crystalline objects, chain folding, lamellar crystalline growths and lattice dislocations. Recently, Lopez-Rubio et al. (2008a) suggested that a more appropriate way of approaching the crystalline structure of starch is to use the crystal-defect concept as opposed to the two-phase concept. According to the crystal defect concept of polymer structure, a portion of the X-ray scattering from the crystalline domains is diffuse and contributes to the so-called amorphous background.

\subsection{Starch properties}

\subsubsection{Granular swelling}

Most starches are insoluble in cold water and undergo a limited reversible swelling due to diffusion and absorption (exothermic process) of small amounts of water into the amorphous region (French, 1984). In the presence of excess water, during heating, the starch granule swells and its volume increases. During this process, crystalline structures of starch molecules are disrupted by increased hydrogen bonding between water molecules and hydroxyl groups, and 
this induces granular swelling (Tester and Karkalas, 1996). Therefore, hydration and swelling of starch during heating reflect the magnitude of interaction between the starch chains within the amorphous and crystalline domains. The amylose to amylopectin ratio and the molecular weight/distribution of amylose and amylopectin may affect the extent of this interaction, resulting in variation in the swelling power and solubility of the starch (Gomand et al., 2010; Karim et al., 2007; Ratnayake et al., 2002). Tester and Morrison (1990a) proposed that the swelling property of starch granules is mainly influenced by amylopectin, while amylose acts both as a diluent and as an inhibitor. Furthermore, it has been well documented that minor components of starch, such as phospholipids, monoacylglycerols, phosphate monoesters (Karim et al., 2007; Srichuwong et al., 2005; Roach and Hoseney, 1995; Tester and Morrison, 1990a) and protein (Wang and Seib, 1996), also have enormous effects on swelling properties. Difference in the morphological structures of granules may also be responsible for differences in swelling power and solubility (Singh and Singh, 2001). Sasaki and Matsuki (1998) showed that starches with higher swelling power tended to contain higher proportions of long amylopectin chains ( $\mathrm{dp} \geq 35$ ). However, Goman et al. (2010) have shown in studies on potato and cassava starches that longer amylopectin chains $(\mathrm{dp}>18)$ inhibit swelling, whereas short chains $(\mathrm{dp}<14)$ favour swelling.

In general, starches from legumes, roots and tubers exhibit a single stage swelling (Hoover, 2001; Hoover and Sosulski, 1986), whereas normal cereal starches show a two stage swelling (Langton and Hermansson, 1989; Doublier et al., 1987; Leach et al,, 1959). Many studies showed that potato starches have higher swelling power than starches from other botanical sources (Eliasson and Gudmundsson, 1996; Swinkels, 1985; Vandeputte et al., 2003). This has 
been attributed to the presence of a higher amount of phosphate ester groups on potato amylopectin (Galliard and Bowler, 1987). Waxy starches usually swell to a greater extent than their normal counterparts. The faster swelling of amylose-free starches has been observed for waxy potato (Gomand et al., 2010; Luo et al., 2009; McPherson and Jane, 1999; Visser et al., 1997), waxy barley (Tester and Morrison, 1990b), waxy maize (Li et al., 2001) and waxy rice (Hagenimana and Ding, 2005). The swelling of starch granules is crucial in explaining pasting behaviour and rheological properties (section 2.5.4) (Li and Yeh, 2001).

Starch swelling has been studied by different methods. The classical swelling power (SP) is the ratio of the wet weight of the sedimented gel to its dry weight (Leach et al., 1959). This method shows a disadvantage in that intragranular water cannot be distinguished from intergranular water. Tester and Morrison (1990b) introduced the new concept of swelling factor (SF), which is the ratio of the volume of sedimented gel (volume of swollen granules) to the volume of dry starch granules (initial volume) with a density of $1.4 \mathrm{~g} / \mathrm{ml}$. By this technique, the volume of the starch particles is determined from changes in the concentration of a dye, Blue dextran, which by virtue of its high molecular weight $(2,000,000)$ is excluded from the swollen starch granules. Therefore, compared to Leach et al. (1959) method, the Blue dextran method has the advantage of measuring intragranular water (Lopez et al., 2004).

\subsubsection{Amylose leaching}

Amylose leaching mainly occurs at low temperatures, whereas molecules of higher molecular weight leach at higher temperatures (Gomand et al., 2010; Eliasson and Gudmundson, 1996; Ring et al., 1985; Tester and Morrison, 1990). However, amylose can be completely extracted 
from granules only at temperatures above $90^{\circ} \mathrm{C}$ (Banks and Greenwood, 1975). Perez and Bertoft (2010) postulated that these larger amylose chains, which are not easily leached, may participate in double helices with amylopectin (Kasemsuwan and Jane, 1994) or may be entangled within the intricate architecture of the starch granule. In addition to temperature, the extent of amylose leaching has been shown to be influenced by: 1) the extent of interaction between amylose chains (AM-AM) and/or between amylose and outer branches of amylopectin (AM-AMP) (Hoover et al., 2010; Chung et al., 2009; Jayakody et al., 2007; Waduge et al., 2006; Zhou et al., 2004), 2) the amount of lipid-complexed amylose chains (Morrison et al., 1993; Ratnayake et al., 2001; Gunaratne and Hoover, 2002; Nakazawa and Wang, 2004), 3) phosphate content (Gunaratne and Hoover, 2002), and 4) granular size (smaller granules leach more amylose than larger granules) (Lindeboom et al,, 2004).

\subsubsection{Gelatinization}

Starch granules are insoluble in cold water but swell when heated in an aqueous medium. Initially, the swelling is reversible and the optical properties (birefringence) of the granule are retained. But when a certain temperature is reached, starch granules in an aqueous starch suspension undergo an irreversible order-disorder phase transformation termed gelatinization (Donovan, 1979; Lund, 1984). This order-disorder phase transition is associated with the diffusion of water into the granule, hydration and granular swelling, amylose leaching, heat uptake, unravelling and dissociation of double helices, loss of crystalline order and the consequent loss of birefringence, leaching of large molecular weight polymers from the granule (including fragments of amylopectin), and increased viscosity and starch solubilization (Atwell 
et al., 1988, Cooke and Gidley, 1992; Donovan, 1979; Jenkins and Donald, 1998; Sakonidou et al., 2003; Sopade, 1990; Stevens and Elton, 1971).

A wide range of techniques (birefringence end point, viscosity, X-ray diffraction, DSC, iodine blue value, enzymatic digestibility, NMR, light extinction, solubility or sedimentation of swollen granules, and absorption of congo red) have been employed to investigate the gelatinization process (Cooke and Gidley, 1992; Derby et al., 1975; Donovan, 1979; Jenkins and Donald, 1998; Liu, et al., 2002; Sopade, 1990; Stevens and Elton, 1971). Birefringence loss could be detected by the Kofler hot stage microscope with polarized light (Leach, 1965; Liu and Zhoa, 1990; Watson, 1964). The temperature at which granules lose their birefringence is referred to as the gelatinization temperature (Lineback, 1986). The birefringence end point temperature (BEPT, i.e, the temperature at which $98 \%$ of the granules have lost their birefringence) varies among starches of different origin depending on their composition and the structural organization of their granules. Even within a given starch granule population, the gelatinization temperatures of individual granules might span a $10-15^{\circ} \mathrm{C}$ range (Biliaderis, 2009).

Differential scanning calorimetry has been widely used to study the phase transitions of aqueous starch suspensions. The gelatinization process is presented in the DSC as an endothermic peak. DSC measures gelatinization transition temperatures (onset [To], peak [Tp] and conclusion [Tc]) and enthalpy $(\Delta \mathrm{H})$ of gelatinization. Gelatinization appears as the result of four processes: (1) cleavage of existing starch-starch-OH bonds (endothermic), (2) formation of starch-solvent-OH bonds (exothermic), (3) the unwinding helix-coil transition of amylopectin helices (endothermic) and (4) the formation of amylose-lipid complexes (Colonna and Buleon, 2010). Gelatinization 
temperatures are influenced by many factors such as moisture content, botanical source of starch, amylose content, lipid content, starch damage, distribution of amylopectin chains, environmental conditions during growth and the presence of ions and solutes (Hoover and Ratnayake, 2002; Jayakody et al., 2005; Lineback, 1986; Noda et al., 1998; Protserov et al., 2001; Stevenson et al.,2006; Tester and Morrison, 1990a; Vandeputte et al., 2003; Visser et al., 1997; Waigh et al., 2000b). Studies on starches with difierent polymorphic structures showed that A-type crystallites melt at higher temperatures than B-type crystallites (Whittam et al., 1990). The gelatinization temperature of most starches is between 60 and $80 \mathrm{C}$ (Copeland et al., 2009). To has been postulated to represent the melting of the weakest crystallites (Larsson and Eliasson, 1991; Nakazawa and Wang, 2003; Wang et al., 1997). Whereas Tc represents melting of crystallites of high stability (Jacobs et al., 1998). However, there is still no consensus with regard to what $\Delta \mathrm{H}$ represents during the gelatinization process. Tester and Morrison (1990a) have postulated that $\Delta \mathrm{H}$ reflects the overall crystallinity (quality and amount of starch crystallites) of amylopectin. Cooke and Gidley (1992) suggested that $\Delta H$ primarily reflects loss of double helical order (melting of double helices) rather than loss of crystalline order. According to Lopez-Rubio et al. (2008), $\Delta \mathrm{H}$ reflects melting of imperfect amylopectin-based crystals with potential contributions from both crystal packing and helix melting. Reported gelatinization enthalpies of native starches are generaily in the range of $5-20 \mathrm{~J} / \mathrm{g}$ (Biliaderis, 2009).

Starch gives a biphasic endotherm during gelatinization at low moisture levels (water:starch volume fractions less than 0.45) (Donovan, 1979; Evans and Haisman, 1982; Nakazava et al., 1984). Donovan (1979) suggested that in the presence of less water, the endothermic transition 
is characterized as a solvent-aided melting of crystallites in the starch granules. The high temperature transition is due to melting of crystallites without adequate moisture. When excess water is present, the high temperature transition disappears (Donovan, 1979). Evans and Haisman (1982) proposed the "cooperative melting theory", which explains the bi-phasic endotherm as a result of crystallite stability within the granules. The granules containing the least stable crystallites start to change first upon heating. Water absorption by the granules lowers the melting points of remaining crystallites. This cooperative process happens quickly when there is sufficient water and gives a narrow or single DSC endotherm. At low moisture content, there is insufficient water for cooperative melting to take place (Evans and Haisman, 1982).

Several gelatinization mechanisms have been proposed to describe the changes occurring during heating of starch granules in excess water (Biiladeris, et al., 1986; Blanshard, 1987; Donovan, 1979; Evans and Haisman, 1982; Liu et al., 2002; Marchant and Blanshard, 1978; Waigh et al., 2000b). Donovan (1979) proposed a model for gelatinization in excess water whereby the swelling of the amorphous regions of the granule facilitated the "stripping" of starch chains from the surface of crystallites, thereby disrupting crystalline order. Another approach was developed by Slade and Levine (1988) who introduced the glass transition concept as a key event determining the change in polymer mobility. When the temperature increases, molecular motion is initiated, enabling molecules to slide past one another. At this point, the polymer becomes rubbery and flexible. This physical event reflects an increase in the mobility of short segments (3-20) on the polymer backbone and it is called the glass transition temperature $(\mathrm{Tg})$. Below $\mathrm{Tg}$, segmental mobility of polymer chains is frozen in a random conformation, rendering starch 
phases solid and glassy (Slade and Levine, 1988). According to this concept, a glass transition of the amorphous regions will precede the melting of crystallites and gelatinization is controlled by the molecular mobility in the amorphous phase surrounding crystallites (Biliaderis, 1992; Blanshard, 1987).

Waigh ef al. (2000b) proposed a mechanism for gelatinization based on a side chain liquid crystalline polymeric (SCLCP) model of starch (Donald et al., 2001; Jenkins et al., 1993; Waigh et al., 2000a; Waigh et al., 1998). In this mechanism, depending on the water content and heating rate, gelatinization results from helix-coil transitions and transformations from a glassy nematic phase to a plasticized smectic phase of amylopectin helices. In the SCLCP model, amylopectin is suggested as a structural analogue to a synthetic SCLCP with three distinct components: rigid units (mesogens) corresponding to double helices, flexible spacers and a flexible backbone. In the nematic state, helices are not aligned into lamellae, whereas in the smectic state; as in the granules under native conditions, the mesogens are aligned which leads to a $9 \mathrm{~nm}$ repeat between the lamellar lengths.

According to the SCLCP model, the structural phase transitions (mesophases) during gelatinization can be summarized by considering three order parameters: helical ordering (the tendency of helices to line up), lamellar ordering (the amplitude of a sinusoidal density modulation) and the number of helices. At low water content $(<5 \%$, w/w), amylopectin helices are in a glassy nematic state and rigid crystalline parts (mesogens) are somewhat disordered and the single peak corresponds to a helix-coil transition. 
Fig 2.17 A proposed model for the gelatinization mechanism.

a) The single stage process in the gelatinization of starch at low water contents.

b) The two stage process involved in the gelatinization of starch in limited water.

c) The two stage process involved in the gelatinization of starch in excess water. The first stage involves a slow dissociation of the helices side-by-side. Immediately, a helixcoil transition occurs as a secondary effect (Waigh et al., 2000b, Copyright Elsevier, reproduced with permission). 
a) Low water content gelatinization

b) Intermediate water content gelatinization

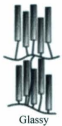

Glassy nematic

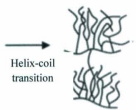

Amorphous
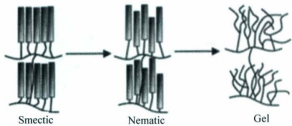

c) Excess water content gelatinization

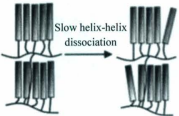

Smectic

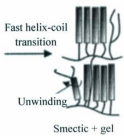


At intermediate water content $(>5 \%,<40 \%$, w/w), the first DSC endotherm is attributed to dislocations between double helices leading to a smectic-nematic transition. The second endotherm is the helix-coil transition with irreversible disentanglement of double helices. Two different processes are shown for A-and B-type starches. It is proposed that the intermediate phase is determined by the length of the amylopectin helices. B-type starch has a much longer amorphous backbone than A-type starch, and because of this, the isotropic structure is formed over gelatinization in the intermediate state, whereas for A-type starch, this takes place during the nematic phase. In excess water $(>40 \%)$, lamellar break-up and disentanglement of double helices occur simultaneously since free, disassociated helices are unstable (Fig 2.17) (Waigh $e t$ al., 2000b).

\subsubsection{Pasting properties}

Pasting is the phenomenon following gelatinization in the dissolution of starch (Atwell et al., 1988). The granules become increasingly susceptible to shear disintegration as they swell and release soluble material as they disintegrate (Whistler and BeMiller, 1997). The paste that is obtained on gelatinization is a viscous mass consisting of a continuous phase (a molecular dispersion) of solubilized amylose and/or amylopectin and a discontinuous phase of granule remnants (granule ghosts and fragments) (Whistler and BeMiller, 1997). Peak viscosities have been shown to be influenced by several factors, such as granule rigidity, extent of granular swelling, extent of amylose leaching, size distribution of granules, heating rate, shearing rate, stirring rate, heating temperature, starch conceniration, the presence of solutes, friction between swollen granules, phosphate monoester content, the proportion of long amylopectin branch chains and the botanical source of the starch (Eerlingen et al., 1997, Evans and Haisman, 1979; 
Hagenimana and Ding, 2005; Hoover and Vasanthan, 1994b; Jane et al.,1999; Jayakody et al.,2007; Ziegler et al., 1993). The pasting profile of starch is commonly monitored using a Rapid Visco ${ }^{\mathrm{TM}}$ Analyser (RVA), which is a heating and cooling viscometer configured especially for testing starch-based and other products requiring precise control of temperature and shear (Hagenimana and Ding, 2005).

\subsubsection{Retrogradation}

When a gelatinized starch suspension is cooled, it begins to retrograde. Retrogradation has been defined as the process which occurs when the molecular chains in gelatinized starches begin to reassociate (hydrogen bonding between starch chains) in an ordered structure (Atwell et al., 1998; Hoover, 2001). During retrogradation, amylose forms double-helical associations of 40-70 glucose units (Jane and Robyt, 1984), whereas amylopectin crystallization occurs by reassociation of the outermost short branches (Ring et al., 1987). Retrogradation is accompanied by increases in crystallinity, gel firmness and turbidity, and the appearance of a B-type X-ray diffraction pattern (Hoover, 2010). Miles et al. (1985) have shown that the short-term development of gel structure and crystallinity in starch gels is dominated by irreversible $\left(\mathrm{T}<100^{\circ} \mathrm{C}\right)$ gelation and crystallization within the amylose matrix, whereas long-term increases in the modulus of starch geis were linked to a reversible crystallization (within gelatinized granules) involving amylopectin. Miles et al. (1985) postulated that crystallization increases granule rigidity and thus enhances their reinforcement of the amylose matrix. The retrogradation of amylose in processed foods is considered to be important for properties related to stickiness, ability to absorb water and digestibility, whereas retrogradation of amylopectin is probably a 
more important determinant in the long-term of quality changes in foods such as staling of bread and cakes (Copeland et al., 2009; Miles et al., 1985; Ring et al., 1987).

It has been suggested that the process of retrogradation can be viewed as a temperaturedependent, three-step sequential mechanism of nucleation, propagation, and maturation (Levine and Slade, 1988, 1989). In retrograded starch, the enthalpy value $(\mathrm{AH})$ provides a quantitative measure of the energy transformation that occurred during the melting of recrystallized amylopectin (Karim et al,, 2000, 2007). The endothermic peak of starches after gelatinization and storage at $4^{\circ} \mathrm{C}$ appears at lower transition temperatures. Starch retrogradation enthalpies are usually $60-80 \%$ smaller than gelatinization enthalpies and transition temperatures are $10-26^{\circ} \mathrm{C}$ lower than those for gelatinization of starch granules (Baker et al., 1998; White et al,, 1989; Yuan et al., 1993). The extent of the decreases in transition temperatures and enthalpy is higher in stored potato starch gels than in corn, rice and wheat gels. This may be attributed to the higher tendency of the potato starch gels toward retrogradation (Singh et al, 2002). Thermal properties of starches after gelatinization and during refrigerated storage could be influenced by the amylose to amylopectin ratio, size and shape of the granules, presence/absence of lipids, and chain length of amylose and amylopectin (Perera and Hoover, 1999; Singh et al., 2003). A greater amount of amylose has generally been linked to a greater retrogradation tendency in starches (Whistler and BeMiller, 1996).

\subsubsection{Acid hydrolysis}

Acid modification is widely used in the starch industry to produce thin boiling starches for use in the food, paper, textile and other industries (Hoover, 2000). Acid hydrolysis of starches has been 
shown to alter granule morphology and crystallinity, gelatinization temperature, gelatinization enthalpy, and viscoelasticity. Several authors have shown that acid hydrolysis could be used as a probe to understand the inner structure of starch granules (Genkina et al,, 2009; Hoover, 2010;; Kang et al., 1997; Kim and Ahn, 1996; Osunsam et al., 1989; Shi and Seib, 1992; Virtanen et al., 1993; Wang et al., 2007a and 2007b). This process involves suspending starch in an aqueous solution of hydrochloric acid or sulphuric acid at a certain temperature. Immersion in sulfuric acid $(15 \%)$ at room temperature produces Nägeli amylodextrins (a low molecular weight acidresistant fraction), whereas treatment with hydrochloric acid $(2.2 \mathrm{M})$ at elevated temperatures $\left(30-40^{\circ} \mathrm{C}\right)$ produces Lintnerized starch (a higher molecular weight acid resistant fraction) (Hoover, 2000). In the presence of a strong acid and heat, the glycosidic bonds between monosaccharides in a polysaccharide are cleaved (Hoover, 2000). Two distinct phases are observed in the course of acid hydrolysis of starch as a function of time. The first more rapid phase is attributed to hydrolysis of amorphous regions of the starch granules, whether they be at the surface or in the interior. The second phase is attributed to a slower degradation of less accessible starch molecules present in the crystailine lamellae (Biliaderis et al., 1981; Hoover, 2000; Jacobs et al., 1998; Kainuma and French, 1971; Nakazawa, and Wang, 2003; Robin et al., 1974; Robin et al., 1975; Watanabe and French, 1980).

\subsubsection{Enzyme hydrolysis of starch by $\alpha$-amylase}

\subsubsection{Classification}

Amylases are glycoside hydrolases (GHs), which act upon the $\alpha-(1,4)$ and/or $\alpha$-(1,6)-linkages of starch polymers (Bijttebier et al., 2008; MacGregor et al., 2001). Several GH classification systems exist. One of the simplest classifications is based on substrate specificity. Each enzyme 
is characterized by the Enzyme Commission (EC) number based on the recommendations of the International Union of Biochemistry and Molecular Biology. GHs are characterized by the number EC 3.2.1.x, with the $\mathrm{x}$ representing the substrate specificity or, in some cases, the molecular mechanism or type of linkage (EC 3.2.1.1, $\alpha$-amylase; EC 3.2.1.2, $\beta$-amylase; EC 3.2.1.3, glucoamylase). This classification does not reflect structural or mechanistic characteristics of enzymes (Bijttebier et al., 2008; Henrissat and Davies, 1997).

The classification of $\mathrm{GH}$ families, based on amino acid sequence and structural similarities, is considered the most powerful and common one, since the amino acid sequence of an enzyme gives important structural and mechanistic information about the enzyme (Bijttebier et al., 2008). On this basis, $\alpha$-amylases are grouped into GH family 13 , whereas $\beta$-amylases and glucoamylases are grouped into $\mathrm{GH}$ family 14 and $\mathrm{GH}$ family 15 , respectively. $\mathrm{GH}$ family 13 amylolytic enzymes include $\alpha$-amylase (EC 3.2.1.1), maltogenic (EC 3.2.1.133) and other maltooligosaccharide-producing amylases (e.g. EC 3.2.1.60, EC 3.2.1.98) and debranching enzymes (pullulanase/isoamylase) (Bijttebier et al., 2008). Beta-amylase is inverting exoamylase and hydrolyze the $\alpha-(1,4)$-linkages at the non-reducing ends of starch molecules. They cannot hydrolyse or pass $\alpha$ - $(1,6)$-linkages; its action stops at the branch points. Beta-amylase degrades starch to $\beta$-maitose and $\beta$-limit dextrins (Goesaert et al., 2009). The action of different amylolytic enzymes on starch is illustrated in Figure 2.18.

\subsubsection{Mechanism for alpha-amylase action}

Enzymatic hydrolysis of glycosidic bonds is carried out with one of two stereochemical outcomes, net retention or net inversion of anomeric configuration. Thus, glycosidases are 
Fig 2.18 Schematic representation of the action of different amylolytic enzymes on starch (amylopectin) polymers. The gray ring structure represents a reducing glucose residue. (A) Endo-type action of alpha-amylase, yielding branched and linear low molecular weight (LMW) dextrins; (B) mainly exo-type action of maltogenic alpha-amylase, yielding mainly maltose; (C) debranching enzyme action, yielding linear dextrins; (D) purely exo-type action of beta-amylase, yielding maltose and beta-limit dextrins; (E) purely exo-type action of glucoamylase, yielding glucose (Goesaer et al,, 2009, Copyright Elsvier, reproduced with permission). 
A CONVENTIONAL ALPHA-AMYLASE
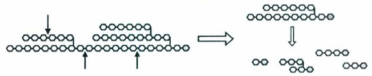

B

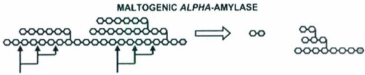

C

$$
\text { PULLULANASEJSOAMYLASE }
$$

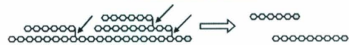

D

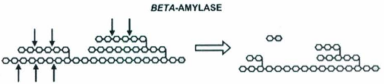

E

GLUCOAMYLASE

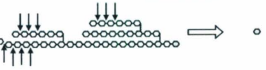


classified as either retaining or inverting (Figure 2.19) (Rye and Withers, 2000). All the enzymes of $\mathrm{GH}$ family 13 retain the anomeric configuration and work according to the double displacement mechanism (MacGregor, 1993; van der Maarel et al., 2002). This is an acid-base catalysed reaction, requiring a proton donor and a nucleophile, in which a glucosyl-enzyme intermediate is formed (Withers and Aebersold, 1995). Takase et al. (1992) have shown that three carboxylic acid groups (one from glutamic acid and two from aspartic acid residues) are essential for catalytic activity. Typically, all endo-acting amylases work according to this action mechanism (Bijttebier et al., 2008). In contrast, exo-acting amylases, such as $\beta$-amylases, are inverting amylases and work through the single displacement mechanism (Henrissat and Davies, 1997). The hydrolysis reaction proceeds through an oxocarbenium ion-like transition state (Oanomeric $\mathrm{C}$ bond has partial double bond character) (Withers and Aebersold, 1995).

\subsection{Double displacement mechanism}

In this mechanism, the carboxylate base initiates cleavage of the bond via nucleophilic attack at C-1 of the glucopyranosyl unit undergoing hydrolysis. Simultaneously, the glycosidic oxygen is displaced and protonated by the carboxylic acid group. The attacking carboxylate group forms a covalent $\beta$-linked acetal ester, giving a glucopyranosyl-enzyme intermediate. Evidence for the covalent glucopyranosyl-enzyme complex was provided for porcine pancreatic $\alpha$-amylase, using cryogenic ${ }^{13} \mathrm{C}-\mathrm{NMR}$ (Tao et al., 1989). This high energy linkage is subsequently hydrolysed by water. Reaction with water is facilitated by the second asparate carboxylate group at the active sites of these enzymes. The carboxylate group abstracts a proton from water, making the water more nucleophilic for its attack on the $\beta$-linked acetal ester. The anomeric carbon atom of the 
Fig 2.19 Proposed mechanisms involved in the hydrolysis of glycosidic linkages (adapted from Robyt, 2009).

a) Double displacement $\left(\mathrm{S}_{\mathrm{N}} 2\right)$, mechanism, giving retention of configuration ( $\alpha$ amylases)

i. Attack on $\mathrm{C}-1$ by a carboxylate group and donation of a proton to the leaving oxygen atom by a carboxyl group

ii. Attack of water on the covalent $\beta$-acetal-carboxyl-ester to give product III with retained configuration at the anomeric end

b) Direct displacement (SN1), mechanism, giving inversion of configuration ( $\beta$ amylases)

i. Direct attack on C-1 by water, assisted by a carboxylate group and donation of a proton to the leaving oxygen atom by a carboxyl group.

ii. Product with inverted configuration

Regeneration of the catalytic groups at the active-site by proton exchange between the two carboxyl groups. 


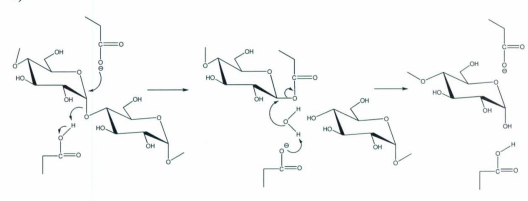

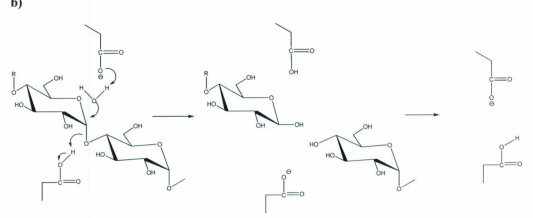


resulting glucopyranosyl unit that is released from the enzyme complex thus has the $\alpha$ configuration, giving retention of the configuration of the product at its reducing end (Robyt, 2009).

\subsubsection{Mode of action of a-amylase}

Alpha-amylases have a multiple attack mechanism (Robyt and French, 1970). In this mechanism, an amylase cleaves several glycosidic bonds after the first random hydrolytic attack and then dissociates from the substrate. The direction of multiple attack is from the reducing to the non-reducing end of the molecule (Robyt and French, 1970). Porcine pancreatic $\alpha$-amylase (endo-amylase, EC 3.2 .1 .1 ) has five subsites to bind substrate. Each subsite binds a glucose unit. It requires binding to at least three giucose units before cleaving an $\alpha-(1,4)$ glycosidic linkage. Both amylose and amylopectin are hydrolysed by virtue of binding of their five glucose residues adjacent to the terminal reducing glucose unit to specific catalytic subsites of the $\alpha$-amylase, followed by cleavage between the second and third $\alpha-(1-4)$ linked glucosyl residue. The final hydrolysis products from $\alpha$-amylase digestion are mainly maltose, maltotriose and maltotetraose (Gray, 1992). Alpha-amylases have no specificity for $\alpha-(1,6)$ branch linkages in amylopectin. Therefore, their capacity to break $\alpha-(1,4)$ links adjacent to the branching point is decreased, mainly due to steric hindrance (Oates, 1997).

\subsubsection{Stages of enzymatic reaction}

Enzymatic reaction consists of several successive phases: diffusion of the enzyme molecule toward the surface of the solid phase and then inside the granuies, adsorption of enzymes on the substrate and finally catalytic reaction (Bird et al., 2009; Dhital et al., 2010; Gallant et al., 1992; 
Sujka and Jamroz, 2007). All three stages, diffusion, adsorption and catalysis, are influenced by enzyme-related factors and by the intrinsic properties of the granules. The diffusion is considered as the limiting step of hydrolysis with regard to the macromolecular nature of amylases and the porosity of substrates or associated products in foods. Thus, the surface area accessible to the enzyme and the efficiency of adsorption onto this surface are important kinetic parameters (Bertoft and Manelius, 1992). Smaller granules, by virtue of their higher available surface area per unit mass, facilitate diffusion and adsorption of enzymes, and thus are catalyzed more rapidly than larger granules (Colonna et al., 1992). Adsorption of amylase on the granule is a pre-requisite for subsequent hydrolysis of starch (Slaughter et al., 2001). Upon adsorption, specific forces between the enzyme and the binding sites of the substrate are formed, leading to an enzyme-substrate complex. The number of adsorption sites for the enzyme is essentially dependent on the porosity and accessibility of the substrate (Colonna et al., 1992). The enzymecatalyzed hydrolysis of $\alpha$ - $(1,4)$ glucosidic bonds involves the enzyme-induced ring distortion of one of the D-glucosyl residues from the ${ }^{4} \mathrm{C}_{1}$-chair conformation to a 'half chair' conformation (Fig 2.20) (Oates, 1997). Ring distortion decreases the enthalpy of activation and increases the susceptibility of the glycosyl residues. Therefore, chains with restricted mobility, either complexed or crystallized, will be hydrolyzed less readily (Oates, 1997). All of these studies showed that hydrolysis of granular starch can be controlled by influencing each of these steps.

\subsubsection{Enzyme hydrolysis of native starches}

The rate and extent of amylolytic hydrolysis of granular starches depends on the botanical origin of the starch and the $\alpha$-amylase source (Dona et al., 2010; Gallant et al., 1992; Zhang et al., 2006). Enzyme concentration and reaction time are other factors influencing both rate and extent 
of hydrolysis. For a given enzyme and digestion conditions, the degree of hydrolysis of a specific starch will depend only on its structural and molecular organization (Bird et al., 2009). Susceptibility of starch to enzyme attack is influenced by several factors, such as granule size and available specific surface (Franco et al., 1992, 1998; Knutson et al., 1982; Tahir et al., 2010; Tang et al., 2004), surface porosity and channels (Fannon et al., 1992, 1993; Sujka and Jamroz, 2007; Zhang et al., 2006;), amylose and amylopectin content (Blazeck and Copeland, 2010; Hoover and Manuel, 1995; Hoover and Sosulski, 1985; Holm and Bjorck, 1988; Ring et al., 1988), crystalline structure (Chung et al., 2010; Zhou et al., 2004; Gunaratne and Hoover, 2002), extent of branching (long amylopectin branches/branch density) (Dona et al., 2010; Shrestha et al., 2010), distribution pattern of $\alpha-(1,6)$ branch points between the amorphous and crystalline regions (Gunaratne and Hoover, 2002; Jane et al., 1997), degree of crystallinity (Chung et al., 2009a; Zhou et al., 2004), packing of polymers within granules (dense/loose), helical conformation of amylose molecules (Shrestha et al., 2010), presence of non-starch substances (proteins, lipids and phosphorus) (Hoover and Manuel, 1995; Perera and Hoover, 1998) and inhibitors (Colonna et al., 1992; Zhou et al., 2004). The kinetics of $\alpha$-amylolysis of granular and gelatinized starch are characterized by an initial rapid hydrolysis phase followed by a slower phase. The reduction in the hydrolysis rate is probably caused by product (maltose and maltotriose) inhibition of the $\alpha$-amylase activity (Colonna et al., 1988).

During $\alpha$-amylolysis by bacterial or pancreatic enzymes, the amorphous parts generally are thought to be more rapidly digested than the crystalline zones due to the steric incompatibility of the double helix and the active site of $\alpha$-amyiases (Gallant et al., 1992; Planchot et al., 1995). 
Fig 2.20 An energy profile ( $\Delta \mathrm{H} \mathrm{kcal} / \mathrm{mole}$ ) for the inter-conversion of the chair to a 'half chair" conformation. 


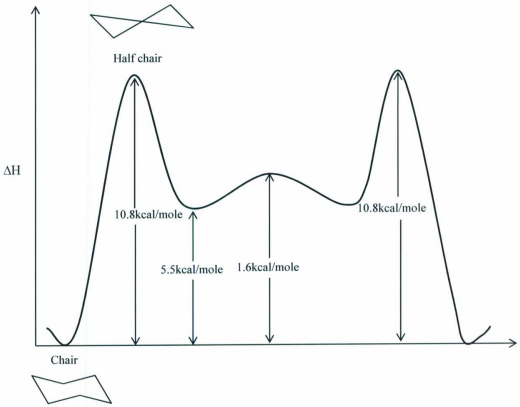


However, Zhang et al. (2006) have shown in studies on cereal starches, that the amorphous and crystalline regions are digested simultaneously. A side-by-side and even digestion mechanism that starts from the channels was proposed as the mechanism leading to the simultaneous digestion of crystalline and amorphous regions. The side-by-side digestion mechanism was attributed to strong association between adjacent amorphous and crystalline layers so that the two regions remained together throughout the digestion process.

The susceptibility of starch granules to enzyme hydrolysis can be classified by the intensity and the manner by which the granules are eroded and corroded (Gallant et al., 1992). In general, enzymes either erode the entire granule surface or sections of it (exo-corrosion/exo-pitting) or digest channels at specific locations on the surface towards the centre (endo-corrosion) (Oates, 1997). Many different modes of enzyme attack have been identified, including pinhole, spongelike erosion, numerous medium sized holes, single holes in individual granules and surface erosion (Sujka and Jamroz, 2007). Planchot et al. (1995) showed that the mode of enzymatic degradation depends on the starch and the enzyme used.

Hydrolysis of native cereal starch begins with enlargement of the surface pores and channels with concurrent hydrolysis from the hilum region toward the outside of the granules, an "insideout" digestion pattern (Zhang et al., 2006). It has been postulated that pores may be the site of initial enzyme attack, openings that allow enzyme molecules direct access to the granule interior (hilum) (Fannon et al., 1993). Planchot et al. (1995) observed that normal and waxy maize starch granules were degraded uniformly, with a large range of degradation levels in the granule population, whereas potato and high-amylose maize starch granules were not equally degraded at 
a given time. B-type starches without pores shows a different hydrolysis pattern, which is referred to as exo-pitting (Gallant et al., 1992). According to Quingley et al. (1998), potato starch hydrolysis is characterized by the formation of a groove along the centre of the granule.

Native cereal starches with A-type crystalline structure (maize) have been shown to be more susceptible to enzymatic hydrolysis than B-type (potato) starches (Planchot et al., 1997). It has been documented that starch granules from waxy maize (Fuwa, Nakajima, Hamada and Glover, 1977), waxy rice (Evers and Juliano, 1976), waxy barley (Fuwa, 1982) and amylose-free sweet potato (Noda et al., 2002) are digested by amylase faster than their normal counterparts, in general. Among non-cereal starches, tapioca starch has relatively higher enzyme susceptibility than other starches such as potato and sweet potato (Zhang and Oates, 1999; Rickard et al., 1991). Potato starch granules are relatively resistant to $\alpha$-amylolysis, with reasons proposed such as crystallinity and thicker blocklets on the surface (Buleon et al., 1998; Gallant et al., 1997, 1992; Tang et al., 2006). However, Dhital et al. (2010) suggested that the dominant reason for the large difference in enzyme hydrolysis between potato and maize starches is the presence of enzyme-accessible channels in maize starch, rather than crystallite polymorph or amylopectin blocklet size differences.

\subsection{Heat-moisture treatment}

Native starches are not widely used in food applications due to their inconsistency and poor functional properties such as low shear and acid resistance, low thermal resistance, thermal decomposition, and high retrogradation tendency (Hoover, 2010). Therefore, modification of starch is often needed to meet industrial demands. In this respect, physical, chemical or 
enzymatic modifications are commonly used to produce starches with desirable properties for specific end uses. Crosslinking and substitution are the two most common chemical modification techniques utilized in the food industry (Luo et al., 2009; Watcharatewinkul et al., 2010).

However, at the present time, physically modified starches prepared by thermal (moisture and heat) or radiation techniques have gained a wider acceptance, especially in the food and pharmaceutical industries (Chung et al., 2009; Hoover, 2010; Watcharatewinkul et al., 2010; Zavareze et al., 2010). Two hydrothermal treatments that modify the physicochemical properties of starch, without destroying granular structure, are annealing and heat-moisture treatment (HMT). Annealing is referred to as treatment of starch in excess water ( $<65 \%)$ or at intermediate moisture $(40-55 \% \mathrm{w} / \mathrm{w})$ for a certain time period at a temperature above the glass transition temperature ( $\mathrm{Tg}$ ) but below the onset temperature of gelatinization (To) (Hoover, 2010; Jacobs and Delcour, 1998). Heat-moisture treatment (HMT) of starches is defined as a physical modification that involves treatment of starch granules at low moisture levels $(<35 \%$ moisture $\mathrm{w} / \mathrm{w})$ over a certain time period $(15 \mathrm{~min}-16 \mathrm{~h})$ and at temperature $\left(80-130^{\circ} \mathrm{C}\right)$ above the glass transition temperature $\left(\mathrm{T}_{p}\right)$ but below the gelatinization temperature (Hoover, 2010; Jacobs and Delcour, 1998; Kulp and Lorenz, 1981; Sair, 1967). HMT has been shown to influence morphology, X-ray diffraction pattern, crystallinity, granule swelling, amylose leaching, gelatinization parameters, viscosity, retrogradation and susceptibility towards acid and $\alpha$-amylase hydrolysis of the starch granule. However, the type and extent of change depends on the botanical origin (e.g. cereal, tuber, root or legume), starch composition (e.g., amyloseamylopectin ratio and lipid content) and treatment conditions (temperature, moisture and 
duration of heating) (Abraham, 1993; Adebowela et al., 2005a, 2005b, 2005c, 2003; Anderson et al., 2002; Anderson and Guraya, 2006; Chung et al, 2009a, 2009b; Brumovsky et al., 2009; Brumovsky and Thompson, 2001; Collado and Corke, 1999; Collado et al., 2001; Donova et al., 1983; Eerlingen et al., 1996, 1997; Franco et al., 1995; Gunaratne and Hoover, 2002; Gunaratne and Corke, 2007; Hoover, 2010; Hoover and Manuel 1996; Hoover and Vasanthan, 1994a, 1994b; Hoover et al., 1994; Jacobs and Delcour, 1998; Kawabata et al., 1994; Kurakake et al., 1996; Kweon et al., 2000; Kulp and Lorenz, 1983; Lawal, 2005; Lawal and Adebowale, 2005; Lim et al., 2001; Lorenz and Kulp, 1981, 1982, 1983; Lu et al., 1996; Moorthy, 1999; Olayinka, et al., 2008; Perera et al., 1997; Perera and Hoover, 1998, 1999; Purwani et al., 2006; Sang and Seib, 2006; Schierbaum and Kettlitz, 1994; Singh et al., 2009; Stute, 1992; Takaya et al., 2000; Vermeylen et al., 2006; Watcharatewinkul et al., 2010; Wongsagonsup, 2009; Wu and Sarko, 1978b; Zavareze et al., 2010).

\subsubsection{Impact of HMT on granule morphoiogy}

HMT temperature has been shown to influence the granule morphology of potato (Kawabata et al., 1994; Vermeylen et al., 2006), maize (Kawabata et al., 1994) and pulse (Chung et al., 2009a) starches. Chung et al. (2009a) observed that HMT at $120^{\circ} \mathrm{C}$ decreased the birefringence intensity and causes loss of birefringence at the granular centre (hilum region). In potato starches, disappearance of birefringence at the granular centre (hilum region) has been shown to increase with increasing HMT temperature (Vermeylen et al., 2006). However, the granule periphery in potato and maize starches was found to remain highiy birefringent even after HMT (Kawabata et al., 1994; Vermeylen et al., 2006). The disappearance of birefringence (loss of radial orientation of AMP chains) at the granular centre has been attributed to the formation of 
voids surrounding the hilum (Vermeylen et al,, 2006). Several studies have shown that HMT has no effect on the size and shape of the starch granule (Adebowala et al., 2005; Gunaratne and Hoover, 2002; Hoover and Vasanthan, 1994a; Hoover and Manuel, 1996; Khunae et al., 2007; Lawal, 2005; Singh et al., 2009; Watcharatewinku et al., 2009).

\subsubsection{Impact of HMT on X-ray pattern and crystallinity}

HMT has been shown to change the X-ray pattern (WAXS) of B-type starches from B-to A+Bor A-type (Donovan et al., 1983; Gunatratne and Hoover, 2002; Kulp and Lorenz, 1981; Sair, 1967; Stute, 1992; Hoover and Vasanthan, 1994; Vieira and Sarmento, 2008). The extent of change has been shown to be influenced by the HMT conditions (temperature, moisture and duration of heating) (Vermeylen et al, 2006). However, Lorenz and Kulp (1982) observed a shift from C-to A-type in arrowroot and cassava starches. The A-type pattern of cereal starches has been shown to remained unchanged on HMT (Donovan et al., 1983; Hoover and Vasanthan, 1994a; Sair, 1967). The change in X-ray pattern of B-type starches on HMT has been attributed to dehydration of the 36 water molecules in the central channel of the B-type unit cell and to the movement of a pair of double helices into the central channel (that was originally occupied by the water molecules) (Fig 2.21) (Gunaratne and Hoover, 2002).

The X-ray intensity of peaks has been shown to decrease in potato (Hoover and Vasanthan, 1994a; Lim et al., 2001; Miyoshi, 2002), cassava (Abraham, 1993), yam (Hoover and Vasanthan, 1994a), barley (Lorenz and Kulp, 1982; Singh et al., 2005), triticale (Lorenz and Kulp, 1982),

pea (Hoover and Manuel, 1996) and bean (Lawal and Adebowale, 2005; Adebowale and Lawal, 2003) starches. However, cereal starches generally exhibit either increased or unchanged 
Fig 2.21 A model of the polymorphic transition from B-type to A-type unit cell in the solid state (adapted from Imberty et al., 1991). 


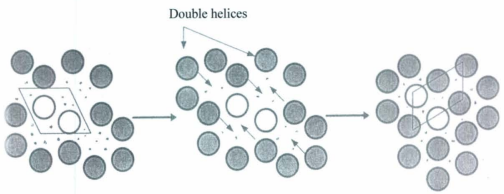

B-type

A-type 
intensities after heat-moisture treatment (Sair, 1967; Donavan et al., 1983; Hoover and Vasanthan, 1994a; Hoover and Manuel, 1996).

The relative crystallinities of potato (Gunartane and Hoover, 2002; Lim et al., 2001; Vermeylen et al., 2006), cassava (Jyothi et al., 2010) pulse (Chung et al., 2009a), rice (Khunae et al., 2007; Zavareze et al., 2010) and maize (Lim et al., 2001) starches have been shown to decrease on HMT. The decrease in relative crystallinity on HMT has been attributed to the disruption of hydrogen bonds between and among double helices (Chung et al, 2009a). An increase in crystallinity has been reported for sweet potato and arrowroot starches subjected to HMT at $120^{\circ} \mathrm{C}$ (Jyothi et al, 2010) and potato starch subjected to HMT at $130^{\circ} \mathrm{C}$ (Vermeylen et al, 2006). Vermeylen et al. (2006) suggested that the breaking of covalent linkages by excessive heating decouples the double helices from the amylopectin backbone and renders them sufficiently mobile to become organized in more perfect/ larger crystallites. The crystallinity and intensity of some A-type tuber starches, such as new cocoyam have been shown, to remain unchanged on HMT (Lawal, 2005). The interaction of free lipids present in native starch granules with amylose chains has been shown to occur during HMT. This is reflected by the decrease in the apparent amylose content of wheat and potato starches on HMT (Hoover and Vasanthan, 1994a; Hoover and Manuel, 1996) and by the increase in intensity of the X-ray diffraction peak centered at $20^{\circ} 2 \theta$ (represents crystalline V-amylose lipid complexes) with an increase in moisture content (due to an increase in mobility of V-amylose-lipid complex chains), which facilitates the formation of larger assemblies that are detectable by $\mathrm{X}$-ray diffraction in rice starches (Zavareze et al., 2010; Khunae et al., 2007). 


\subsubsection{Impact of HMT on granular swelling and amylose leaching}

Regardless of starch origin, HMT reduces granular swelling and amylose leaching of many starches (Chung et al., 2009; Gunaratne et al., 2010; Gunaratne and Hoover, 2002; Hoover et al., 1994; Hoover and Manuel, 1996; Hoover and Vasanthan, 1994a; Hormdok, and Noomhorm, 2007; Olayinka et al., 2008; Sair, 1967; Zavareze et al., 2010). However, in some cereal starches, such as rye, barley, triticale, finger millet and wheat, amylose leaching has been shown to be increased on HMT, inspite of reduced granular swelling (Adebowale et al., 2005a; Kulp and Lorenz, 1981; Kurakake et al., 1996). Furthermore, an increase in amylose leaching has been reported for corn starches subjected to HMT at $120^{\circ} \mathrm{C}$ (Chung et al., 2009b). The increase in amylose leaching on HMT at higher temperatures reflects leaching of both loosely-packed amylose chains and short chains resulting from the disrupted crystallites (occurs mainly at temperatures exceeding $120^{\circ}$ ) (Chung et al., 2009b). The reduction in granular swelling and amylose leaching on HMT has been attributed to amylose-amylose (AM-AM), amyloseamylopectin (AM-AMP), amylopectin-amylopectin (AMP-AMP) interactions (these interactions reduce the number of free hydroxyl groups available for interaction with water), amylose-lipid interactions, crystalline stability, an incrense in crystallinity, and polymorphic transformation $(B \rightarrow A+B)$ [the ingress of water during swelling would be more rapid and higher in the B-type

polymorph, due to double helices being more compactly packed in the A-type polymorph] (Chung et al., 2009a, 2009b; Gunaratne et al., 2010; Gunaratne and Hoover, 2002; Hoover and Vasanthan, 1994b).

\subsubsection{Impact of HMT on gelatinization parameters}

Heat moisture treatment has been shown to increase the gelatinization transition temperatures (onset [To], mid-point [Tp] and conclusion [Tc]) and broaden the gelatinization temperature 
range (To-Tc), whereas the enthalpy of gelatinization $(\Delta \mathrm{H})$ either decreases or remains unchanged on HMT (Chung et al, 2009a, 2009b; Gunaratne and Hoover, 2002; Hoover and Vasanthan, 1994a; Kweon et al., 2000; Vermeylen et al., 2006). The increase in gelatinization temperatures has been attributed to interactions between amylose-amylose (AM-AM), amyloseamylopectin (AM-AMP) chains and amylose-lipid interactions (Gunaratne and Hoover, 2002; Hoover and Vasanthan, 1994a) and polymorphic uransformation $(B \rightarrow A+B)$ and destabilization of crystallites (Gunaratne et al., 2010; Gunaratne and Hoover, 2002). Hoover and Manuel (1996) and Kweon et al. (2000) have shown that the increase in To, Tp and Tc on HMT in normal and waxy maize starches is lower in the latter due to the absence of AM-AM and AMAMP interactions. The reduction in enthalpy of gelatinization on HMT has been attributed to interaction between amylose-amylose (AM-AM) and/or amylose-amylopectin (AM-AMP) chains, decoupling of double helices and disruption of crystallites (Gunaratne et al., 2010; Gunaratne and Hoover, 2002). Gunaratne and Hoover (2002) and Hoover and Vasanthan (1994a) postulated that an increase in the peak temperature is a reflection of melting of crystallites, which are formed because of AM-AM and AM-AMP interactions along the chains, which is stronger in heat-moisture treatments. This suppresses the swelling of the granule, leading to delayed gelatinization and high onset, peak and conclusion temperatures. Furthermore, HMT has been shown to increase the enthalpy associated with melting of the amylose-lipid complex in corn starch (see sec 2.7.2) (Lim et al., 2001).

Several authors showed that the decrease in enthaipy on HMT is more pronounced in tuber starches than in cereal starches (Collado and Corke, 1999; Donavn et al., 1993; Eerlington et al., 1996; Gunaratne et al., 2010; Hoover and Vasanthan, 1994a). This variation could be explained 
by the influence of two factors, namely the packing arrangement of double helices and phosphate monoester content. In B-type starches (potato), the packing of double helices is less compact than in A-type starches (cereal). Furthermore, B-type unit cells contain more water molecules (thirty-six) than A-type unit cells (four) (Wu and Sarko, 1978b). Therefore, double helices of tuber starches would be more mobile and more prone to disruption than those in cereal starches (Gunaratne et al., 2010; Hoover 2010). Tuber starches, such as potato, have been shown to have a higher phosphate monoester content than cereal starches (Hizukuri et al., 1970). The presence of higher phosphate monoester content would hinder strong interaction between double helices (due to repulsion between negatively charged phosphate groups on adjacent amylopectin chains) during HMT in tuber starches (Gunaratne and Hoover, 2002). Hoover and Manuel (1996) have shown in studies on amylomaize (B-type) starches that the $\triangle \mathrm{H}$ remains unchanged on HMT. This suggests that the disruptive effect of HMT on amylopectin is influenced more by the phosphate monoester content than by unit cell structure (Hoover, 2010).

\subsubsection{Impact of HMT on pasting properties}

Heat-moisture treatment of many starches, including potato (Kulp and Lorenz, 1981; Stute, 1992; Hoover and Vasanthan, 1994a; Purwani et al., 2006; Gunaratne and Corke, 2007; Svegmark et al., 2002), sweetpotato (Collado and Corke, 1999; Singh et al., 2005), cassava (Lorenz and Kulp, 1981; Abraham, 1993), yam (Hoover and Vasanthan, 1994a; Moorthy, 1999; Lawal, 2005), cana (Watcharatewinkul et al. 2009; 2010), maize (Chung et al., 2009b; Franco et al., 1995; Hoover and Manuel, 1996; Loisel et al., 2006), rice (Anderson and Guraya, 2006; Shih et al., 2007; Zavareze et al., 2010), sorghum (Olayinka et al., 2008), oat (Hoover and Vasanthan, 1994a), lentil (Hoover and Vasanthan, 1994a), mucuna bean (Adebowale and Lawal, 2003), 
pigeon pea (Hoover et al., 1993), amaranth (Gunaratne and Corke, 2007) has been shown to increase pasting temperature and decrease paste viscosity and breakdown. However, peak viscosity of waxy maize and wheat starches remained unchanged or increased after HMT (Hoover and Manuel, 1996; Hoover and Vasanthan, 1994a). Hoover and Vasanthan (1994a) claimed that structural rearrangement contributed to these changes. Recently, Watcharatewinkul et al. (2010) have shown in studies on canna starches, that pasting properties of HMT (moisture $22 \%$ and $25 \%, 100^{\circ} \mathrm{C}, 16 \mathrm{~h}$ ) starches are equivaient to those of canna starches crosslinked with sodium trimetaphosphate $(0.2$ and $0.5 \%)$.

\subsubsection{Impact of HMT on enzyme hydrolysis}

Several authors have shown that HMT parameters such as moisture content, temperature, duration of heating, botanical origin and starch composition influence the susceptibility of starches towards $\alpha$-amylase (Hoover, 2010; Jacobs and Delcour, 1998). HMT ( $100^{\circ} \mathrm{C}, 30 \%$ moisture, $16 \mathrm{~h}$ ) of pulse starches (field pea, wrinkled pea, pigeon pea, black bean, lentil) (Hoover and Manuel, 1996) and ruber and root starches (potato, taro, new cocoyam, cassava, yam) (Kawabata et al., 1994; Hoover and Vasanthan, 1994a; Perera and Hoover, 1998; Gunaratne and Hoover, 2002) has been shown to increase susceptibility towards $\alpha$-amylase. Lorenz and Kulp (1983) have shown that digestibility of potato starch increased with increasing moisture content prevailing during $\mathrm{HMT}\left(100^{\circ} \mathrm{C}, 16 \mathrm{~h}, 18\right.$ and $27 \%$ moisture content).

In cereal starches, the digestibility of normal and waxy maize starches by a mixture of PPA and amyloglucosidase was shown to decrease on $\operatorname{HMT}\left(100^{\circ} \mathrm{C} / 16 \mathrm{~h}\right)$ at $18 \%$ moisture content (Franco et al., 1995), whereas the digestibility of normal and waxy maize starches increased on 
HMT $\left(100^{\circ} \mathrm{C} / 16 \mathrm{~h}\right)$ at $27 \%$ moisture content (Franco et al, 1995). Hoover and Manuel (1996) showed that digestibility of normal, waxy, and Hylon V maize starches after $72 \mathrm{~h}$ of incubation with pancreatic porcine $\alpha$-amylase (PPA) decreased on HMT $\left(100^{\circ} \mathrm{C} / 16 \mathrm{~h} / 30 \%\right.$ moisture). Kweon et al. (2000) reported that the digestibility of normal and Hylon V (57\% amylose), HylonVII (70\% amylose) and waxy maize starches by PPA decreased on HMT $\left(110^{\circ} \mathrm{C} / 16 \mathrm{~h}\right)$ at $15-21 \%$ moisture content. However, starch digestibility increased on HMT $\left(110^{\circ} \mathrm{C} / 16 \mathrm{~h}\right)$ at moisture contents in the range $24-27 \%$ in normal and Hylon V starches, and at $27 \%$ moisture in a waxy and Hylon VII starches. In this study (Kweon et al., 2000), the lowest digestibility was observed after HMT at $18 \%$ moisture content. However, the extent of this increase was most pronounced after HMT at $27 \%$ moisture content. A similar trend has been reported by Zavareze et al. (2010) on HMT $\left(110^{\circ} \mathrm{C}, 1 \mathrm{~h}, 15,20\right.$ and $25 \%$ moisture content) of high, medium and low amylose rice starches hydrolyzed by bacterial $\alpha$-amyiase. Anderson et al. (2002) studied the digestibility of HMT (20\% moisture content) normal and waxy rice starches at their melting temperature and reported that the digestibility decreased by 25 and $10 \%$, respectively, for HMT normal and waxy rice starches relative to untreated controls. However, the extent of hydrolysis of Hylon V and Hylon VII maize starches on HMT at $100^{\circ} \mathrm{C}$ by heat stable bacterial $\alpha$-amylase from Bacillus licheniformis has been shown to decrease with increasing moisture levels (15 to 27\%) (Kweon et al., 2000). The reasons for the different kinetic profiles of amylolysis are likely to be ascribed to variations in the magnitude of interactions between AM-AM, AM-AMP, AMP$\mathrm{AMP}$ and $\mathrm{AM}-$ lipid, polymorphic transformation $(\mathrm{B} \rightarrow \mathrm{A}+\mathrm{B})$, crystallite disruption, crystallite reorientation, and formation of fissures and cracks on the granule surface during HMT (Hoover, 2010). 


\subsubsection{Uses of HMT starches}

HMT starch has important properties for the food industry. HMT has been shown to improve thermal stability and decrease the extent of set-back (Hoover, 2010). Therefore, HMT starches could be utilized in the canned and frozen food industries for their respective advantages (Zavareze et al., 2010). The decrease in granular swelling and amylose leaching, and the increase in the thermal, acid and shear stability that occur on HMT, are all desirable properties for the production of noodles, retort foods (soups and sauces), dressings, baked foods, batter products, confections, dairy products, creams and fat minetics (Hormdok and Noomhorm, 2007; Kurahashi and Yoshino, 2000). Thus, HMT could be used as an alternative to chemical modification (Yoshino et al., 1994).

HMT has been shown to manipulate the digestibility (rate and extent of PPA hydrolysis) of many starches (Hoover, 2010). For nutritional purposes, starch is classified into three general types based on rate of digestion: rapidly digestible starch (RDS), slowly digestible starch (SDS) and resistant starch (RS) (Englyst et al., 1992). Starch that resists digestion is of nutritional importance because of its reduced glycemic and insulinemic responses, as well as its hypocholesterolemic and improved gut health effects (Lim et al., 2005; Sajilata, et al., 2006; Grabitske and Slavin, 2009; Singh et al., 2010). HMT has been used to modify the amount of RDS, SDS and RS in various native starches (Brumovsky and Thompson, 2001; Mutungi, et al., 2009; Sang and Seib, 2006; Shin et al., 2005; Wongsagonsup et al., 2009).

SDS is considered beneficial for dietary management of metabolic disorders, including diabetes and hyperlipidemia (Wolever and Mehling, 2002). A high proportion of SDS relative to RDS in 
a starchy food indicates a food with a low glycemic index (GI). Consumption of foods containing SDS improves overall blood glucose control in patients suffering from diabetes mellitus (Brand et al., 1991) and attenuates total serum cholesterol levels in hyperlipidemic patients (Jenkins et al,, 1987). Therefore, much attention is being given to SDS as a new functional ingredient (Wongsagonsup et al, 2009; Shin et al, 2004). Heat-moisture treatment has been shown to increase SDS in pulse (pea, !entil and navy bean) starches (Chung et al., 2009 c).

RS has been defined as the fraction of starch that escapes digestion in the small intestine of healthy individuals and arrives in the colon, where it is fermented to short chain fatty acids (Englyst and Cummings, 1985). The health benelits of RS have been reported as prevention of colon cancer, hypoglycemic effects, substrate for growth of probiotic microorganisms, reduction of gall stone formation, hypocholesterolemic effects, inhibition of fat accumulation and increased absorption of minerals (Sajilata et al., 2006). This has stimulated research on RS fractions in food products. Several studies showed that HMT increases the RS content in high amylose starches (Brumovsky and Thompson, 2001; Jacobasch et al., 2006; Sang and Seib, 2006). Brumovsky et ai. (2009) have shown in studies on corn, potato, cassava and wheat starches, that HMT increased the boiling-stable RS content in starches of corn, potaio and wheat in relation to the corresponding native starches, but not in cassava starch, which exhibited a decrease in the content of RS after HMT. Further, they reported that corn starch subjected to HMT for 60 minutes at $120^{\circ} \mathrm{C}$ produced the highest content of RS $(4.2 \%$, w/w), followed by potato starch $(3.1 \%$, w/w) with identical treatment. 


\section{CHAPTER 3}

\section{MATERIALS AND METHODS}

\subsection{Materials}

Normal potato starch and crystalline porcine pancreatic $\alpha$-amylase (PPA) (EC 3.2.1.1, type 1A) were purchased from Sigma Chemical Co. (St. Louis, MO, USA). Waxy potato starch (Eliane 100) was a gift from Louise Lynch (Chemroy Canada, Inc., Laval, QC, Canada). APTS (8aminopyrene-1,3,6-trisulfonic acid, trisodium salt), sodium cyanoborohydride and Pro-Q Diamond phosphoprotein gel stain were purchased from Molecular Probes, Eugene, OR, USA. All chemicals and solvents were of ACS certified grade.

\subsection{Methods}

\subsubsection{Chemical composition}

\subsubsection{Moisture content}

The moisture contents of starch samples were quantitatively determined according to Method 44-15A of the AACC (2000).

\subsubsection{Nitrogen content}

The nitrogen content was determined by the Micro Kjeldahl method (AACC, 2000).

\subsubsection{Phosphorous content}

The phosphorus contents of potato starches were determined as phosphomolybdic Blue complexes according to the procedure described by Morrison (1964). Starch (5 mg, db) was placed in a hard glass test tube $(15-20 \mathrm{~mL}$ capacity) accurately calibrated at the $5 \mathrm{~mL}$ level. 
Sulphuric acid $(0.3 \mathrm{~mL}, 98 \%)$ was added and the contents were gently heated over a small micro bumer flame until charring was completed, and the climbing film of acid on the walls of the tubes was no longer viscous with partially charred organic matter. One drop of hydrogen peroxide $\left(\mathrm{H}_{2} \mathrm{O}_{2}, 30 \%\right.$, w/v) was then added to contact the walls of the tubes just above the acid, and the tubes were shaken. (If the acid was not clear, the process was repeated using one drop of peroxide at a time). When clear, the tubes were gently boiled for $1 \mathrm{~min}$ and then allowed to cool. The contents were diluted to approximately $4 \mathrm{~mL}$ with deionized water to wash down the walls of the tubes. Sodium sulphite $\left(\mathrm{Na}_{2} \mathrm{SO}_{3} .7 \mathrm{H}_{2} \mathrm{O}, 33 \%\right.$, w/v) solution $(0.1 \mathrm{~mL})$ was then added and the tubes shaken to acidify the lower walls of the tube. Ammonium molybdate $\left[\left(\mathrm{NH}_{4}\right)_{6} \mathrm{Mo}_{7} \mathrm{O}_{24}, 4 \mathrm{H}_{2} \mathrm{O}, 2 \%\right.$, w/v $]$ solution $(1 \mathrm{~mL})$ was added directly into the acid, (taking care not to touch the walls of the tube) followed by the addition of ascorbic acid $(0.001 \mathrm{~g})$. The tubes were then heated at $100^{\circ} \mathrm{C}$ for $10 \mathrm{~min}$ and cooled. The volume of the solution was adjusted to 5 $\mathrm{mL}$, the tube was stoppered and shaken, and the optical density at $822 \mathrm{~nm}$ was measured against water. The optical density of the blank was also determined at $822 \mathrm{~nm}$. A standard curve (Appendix 4 ) of phosphorus $(\mu \mathrm{g})$ versus optical density $(822 \mathrm{~nm})$ was prepared using a standard phosphorus solution (1.0068 $\mathrm{g} \mathrm{NaH}{ }_{2} \mathrm{PO}_{4} \cdot 2 \mathrm{H}_{2} \mathrm{O} / \mathrm{L}$ contains $200 \mu \mathrm{g} / \mathrm{mL}$ ) and used to calculate the phosphorus content of the samples.

\subsubsection{Ash content}

The ash contents of starch samples were determined by Method 08-17 of the AACC (2000). 


\subsubsection{Lipid Content}

\subsection{Surface Lipid}

The surface lipid contents of the starch samples were determined according to the procedure described by Vasanthan and Hoover (1992b). The lipids were extracted at ambient temperature $\left(25-27^{\circ} \mathrm{C}\right)$ by mixing a starch sample $(5 \mathrm{~g}, \mathrm{db})$ with $100 \mathrm{~mL}$ of chloroform/methanol $(2: 1$, v/v). The contents were mixed thoroughly for $1 \mathrm{~h}$ and the solution was filtered carefully using Whatman No. 4 filter paper into a $250 \mathrm{~mL}$ round bottom flask. The residue was thoroughly washed out with chloroform/methanol solution. The lipid solvent mixture was evaporated to dryness using a rotary evaporator (Rotovapor-R110, Büchi Laboratorimus-Technik AG, Flawill/Schweiz, Switzerland). The crude lipid extracts were purified by the method of Bligh and Dyer (1959) before quantification.

\subsection{Bound Lipid}

Bound lipids in the starch samples also were determined according to the method of Vasanthan and Hoover (1992b). The residues from the chloroform/methanol extractions were refluxed with $n$-propanol:water $(3: 1, \mathrm{v} / \mathrm{v})$ in a Soxhlet apparatus at $85^{\circ} \mathrm{C}$ for $7 \mathrm{~h}$. The solvent was evaporated to dryness using a rotary evaporator and the crude lipid residue was purified by the method of Bligh and Dyer (1959) before quantification.

Crude lipid purification (Bligh and Dyer, 1959; Vasanthan and Hoover, 1992b):

The crude lipid extracts were purified by extraction in a separatory funnel with chloroform: methanol:water $[1: 2: 0.8(\mathrm{v} / \mathrm{v} / \mathrm{v})]$. The round bottom flask containing the crude lipid extract was washed with chloroform:methanol:water mixture and the washings were transferred to separatory funnel and chloroform and water were added further to form a biphasic layer. The heavy 
chloroform layer at the bottom of separatory funnel, which contained the purified lipid, was withdrawn into a pre-weighted round bottom flask and evaporated to dryness in a rotary evaporator. The round bottom flask with purified lipid was then removed and the contents dried at $60^{\circ} \mathrm{C}$ for $\mathrm{I} \mathrm{h}$ in forced-air oven. The dried lipid was cooled to room temperature in a desiccator and weighed.

$$
\operatorname{Lipid}(\%)=\frac{W_{2}-W_{1}}{W_{0}} \times 100
$$

Where,

$\mathrm{W}_{0^{-}}$sample weight $(\mathrm{g}, \mathrm{db})$

$\mathrm{W}_{\mathrm{L}}$ - weight of tare round bottom flask $(\mathrm{g})$

$\mathrm{W}_{2}$ - weight of round bottom flask and lipid after drying $(\mathrm{g})$

\subsubsection{Amylose content}

The amylose contents of normal and waxy potato starches were determined by the colorimetric method of Hoover and Ratnayake (2004).

\subsection{Apparent amylose content}

Starch ( $20 \mathrm{mg}, \mathrm{db}$ ) was weighed into a round bottom, screw cap tube and solubilized by adding dimethylsulfoxide ( $90 \%$ DMSO, $8 \mathrm{~mL}$ ). The contents were vortexed vigorously and heated for $15 \mathrm{~min}$ in a water bath at $85^{\circ} \mathrm{C}$ with intermittent mixing. The tubes were then allowed to cool at 
room temperature and diluted to $25 \mathrm{~mL}$ in volumetric flask. One millilitre of the diluted solution was mixed with water $(40 \mathrm{~mL})$ and $\mathrm{I}_{2} / \mathrm{KI}$ solution $\left(5 \mathrm{~mL},\left[0.0025 \mathrm{M} \mathrm{I}_{2}\right.\right.$ and $0.0065 \mathrm{M} \mathrm{KI}$ mixture]) and vortexed. The final volume was adjusted to $50 \mathrm{~mL}$ in a volumetric flask and the contents were allowed to stand in the dark for $15 \mathrm{~min}$ at room temperature. The absorbance measurements were taken at $600 \mathrm{~nm}$ using a UV-visible spectrophotometer and the amylose contents of the samples were calculated from the standard curve obtained with pure amylose and amylopectin mixtures, over the range of $0 \%$ to $100 \%$.

\subsection{Total amylose content}

Defatted starch was prepared by Soxhlet extraction with $75 \%$ aqueous $n$-propanol at $85^{\circ} \mathrm{C}$ for $7 \mathrm{~h}$ and the amylose content of the defatted starch was determined by the procedure described above.

\subsection{Heat-moisture treatment (HMT)}

Starch samples were equilibrated at room temperature to a water activity of 0.97 in a desiccator over saturated $\mathrm{K}_{2} \mathrm{SO}_{4}$ solution for up to one month. Following equilibration, the moisture contents $(\sim 27 \%)$ of the samples were determined by AACC $(2000)$. The starch samples were then heated at $80,100,120$ and $130^{\circ} \mathrm{C}$ for $16 \mathrm{~h}$ in a forced-air oven. The HMT starches were subsequently air dried to a uniform moisture content $(\sim 10 \%)$. In the text of this thesis, normal and waxy potato starches in their native state are referred to as NP and WP, respectively, whereas NP80, NP100, NP120 NP130, WP, WP80, WP100, WP120 and WP130 represent NP and WP starches after HMT at $80,100,120$ and $130^{\circ} \mathrm{C}$. 


\subsection{Polarized light microscopy}

Native and HMT starch suspensions (water-glycerol, 50:50, v/v) were observed under bright field light and crossed polarized light (magnification $400 \times$ ) using a binocular microscope (Nikon Microscope, Eclipse 80i) equipped with real time viewing (Q-capture Pro ${ }^{\mathrm{TM}}$ ). A Qlmaging digital camera (QICAM fast 1394, Canada) was used for image capture.

\subsection{Scanning electron microscopy (SEM)}

The granule morphologies of native and HMT normal and waxy potato starches were analyzed before and after amylolysis ( $24 \mathrm{~h}$ ) using a Hitachi S570 scanning electron microscope (Nissei Sangyo Inc., Rexdale, ON, Canada) at an accelerating potential of $15 \mathrm{kV}$. Dry starch samples were brushed onto the surface of double-sided carbon adhesive tape mounted on an aluminium stub and then coated with a thin film $(20 \mathrm{~nm})$ of gold in an argon atmosphere.

\subsection{Confocal laser scanning microscopy (CLSM)}

The molecular distribution of amylose and amylopectin, morphological changes during HMT and the degradation pathway of PPA into the granule interior were visualized by staining with APTS (Molecular Probes, Eugene, OR, USA) according to the method described by Blennow et al. (2003). Phosphorus was stained with Pro-Q Diamond stain (Molecular Probes) based on the method of Glaring et al. (2006). Starch samples (10-15 mg) were stained in $10 \mu \mathrm{L}$ of freshly made APTS solution (20 mM APTS in $15 \%$ acetic acid) and $10 \mu \mathrm{L}$ of $1 \mathrm{M}$ sodium cyanoborohydride at $30^{\circ} \mathrm{C}$ for $15 \mathrm{~h}$. The APTS-stained starch granules were washed five times with deionized water and then dispersed in $0.5 \mathrm{~mL}$ of Pro-Q Diamond solution at room 
temperature for $1 \mathrm{~h}$. After thoroughly washing five times with deionized water, the stained starch granules were suspended in $0.5 \mathrm{~mL}$ of $50 \%$ glycerol for CLSM observation.

Stained starch granules in $50 \%$ glycerol $(10 \mu \mathrm{L})$ were dropped into a glass bottom culture dish (MatTek Corporation, Ashland, MA, USA), mixed with $0.1 \mathrm{~mL}$ of deionized water, covered with a glass slip, and then visualized with a confocal laser scanning microscope (Zeiss LSM 710, Carl Zeiss Microlmaging GmbH, Jena, Germany) equipped with a 40x/1.3 oil objective lens. The excitation wavelength achieved with a diode laser was $488 \mathrm{~nm}$ for APTS and $561 \mathrm{~nm}$ for Pro-Q Diamond with an emission light interval of $490-560 \mathrm{~nm}$. Laser power capacity and master gain were adjusted to maximum saturation. Images of optical sections of starch granules were recorded with ZEN 2009 software (Carl Zeiss Microlmaging GmbH). For each starch sample, a population of granules was examined with 15-20 images, with approximately 10-15 granules in each image.

\subsection{Iodine binding}

The ability of iodine to complex starch chains before and after HMT was determined by equilibrating starch samples at $100 \% \mathrm{RH}$ for $2 \mathrm{~h}$. To determine iodine binding, a thin layer of

the equilibrated starch sample $(0.1 \mathrm{~g})$ was spread on a plastic dish and exposed for $24 \mathrm{~h}$ at room temperature to iodine vapour generated from $2 \mathrm{~g}$ of iodine crystals placed in the desiccator containing the starch samples. The desiccator was covered with aluminum foil to avoid light exposure. The reflectance spectra of iodine stained starches were determined using the Kubelka and Munk (1931) equation:

$$
\frac{K}{S}=\frac{(1-R)^{2}}{2 R}
$$


where $\mathrm{K}$ is an absorption coefficient, $\mathrm{S}$ is a scattering coefficient representing the fraction of light that is scattered when the incident light strikes a sufficiently thick layer of starch granules, and $\mathrm{R}$ is the reflectance expressed as fraction between 0 and 1 . The $\mathrm{K} / \mathrm{S}$ value of the starches (represents the absorption corrected to the light scattering) was measured over the wavelength range of 360 to $740 \mathrm{~nm}$ (CM 3500-d spectrophotometer, Konica Minolta, Mahwah, NJ, USA) at $10 \mathrm{~nm}$ intervals.

\subsection{Fluorophore-assisted capillary electrophoresis (FACE)}

The AMP chain length distributions of native and HMT starches were analysed by FACE as described by Morell et al. (1998). Starches were debranched (at $37^{\circ} \mathrm{C}$ for $2 \mathrm{~h}$ ) with isoamylase (10U) and labelled with APTS (Fig 3.1) (O'Shea et al., 1998). FACE was conducted using the Beckman Coulter Proteome Lab PA800 equipped with $488 \mathrm{~nm}$ laser module. The N-CHO (PVA) capillary with a preburned window ( $50 \mu \mathrm{m} \mathrm{ID} \mathrm{and} 50.2 \mathrm{~cm}$ total length) was used for separation of debranched samples. Maltose was used as an internal standard. Separation was conducted at $10^{\circ} \mathrm{C}$ for $30 \mathrm{~min}$. Data was recorded and analysed using 32-Karat software. The degree of polymerization, (DP) was assigned to peaks based on the migration time of maltose. 
Figure 3.1 Labelling reaction of glucose with 8-aminopyrene-1,3,6-trisulphonic acid (APTS) (adapted from O'Shea et al., 1998). 


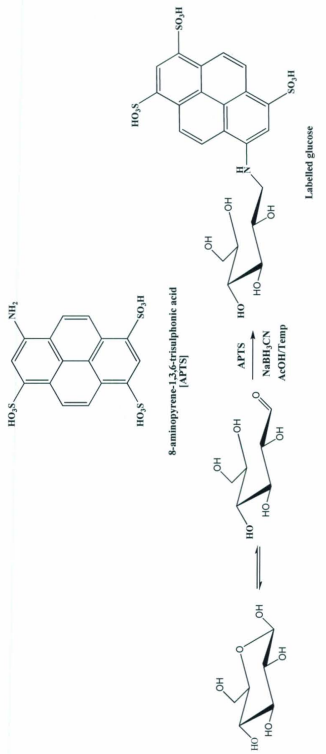




\subsection{Attenuated total reflectance Fourier transform infrared spectroscopy (ATR-FTIR)}

The ATR-FTIR spectra of native and HMT starches were recorded on a Digilab FTS 7000 spectrophotometer (Digilab USA, Randolph, MA, USA) equipped with a thermoelectrically cooled deuterated triglycine sulphate (DIGS) detector using an attenuated total reflectance (ATR) accessory at a resolution of $4 \mathrm{~cm}^{-1}$ and 128 scans. Spectra were base-line-corrected and then deconvoluted by drawing a straight line between 1200 and $800 \mathrm{~cm}^{-1}$ (using Win-IR Pro software). A half-band width of $15 \mathrm{~cm}^{-1}$ and a resolution enhancement factor of 1.5 with Bessel apodization were employed. Intensity measurements were performed on the deconvoluted spectra by recording the peak height of the absorbance bands from the base-line.

\subsection{Wide angle $\mathrm{X}$-ray diffraction (WAXS)}

Native and HMT normal and waxy potato starches were kept in a desiccator over saturated $\mathrm{K}_{2} \mathrm{SO}_{4}$ solutions ( $\mathrm{a}_{\mathrm{w}}-0.97$ ) at room temperature for 14 days prior to $\mathrm{X}$-ray diffraction measurements. The hydrated native and HMT starches were then packed tightly into a round aluminum holder. X-ray diffractograms were obtained with a Rigaku Ultima IV X-ray diffractometer with operating conditions of target voltage, $40 \mathrm{kV}$, current, $44 \mathrm{~mA}$, scanning range, $3-35^{\circ}$, scan speed, $1.00^{\circ} / \mathrm{min}$, step time, 0.95 , divergence slit width, $0.5^{\circ}$, scatter slit width, $0.5^{\circ}$, sampling width, $0.03^{\circ}$ and receiving slit width, $0.3 \mathrm{~mm}$. IGOR pro 6.1 software (WaveMetrics Inc. Oregon, USA) was used to calculate the relative crystallinity (RC), half height peak width, also called full width at half maximum height (FWHM) using the curve fitting procedure (Appendix 1) described by Lopez-Rubio et al. (2008a). A Gaussian function was used for curve fitting. Peak height and FWHM were calculated after subtracting the amorphous 
background. A- and B-type polymorphic composition of the starches was calculated as described by Ratnayake et al. (2001).

\subsection{Amylose leaching (AML)}

Starch samples $(20 \mathrm{mg}, \mathrm{db})$ in deionized water $(10 \mathrm{~mL})$ were heated at $60-95^{\circ} \mathrm{C}$ in volumecalibrated sealed tubes for $30 \mathrm{~min}$ (tubes were shaken by hand every $5 \mathrm{~min}$ to suspend the starch slurry). The tubes were then cooled to room temperature and centrifuged at $2000 \times g$ for $10 \mathrm{~min}$. The supernatant liquid $(1 \mathrm{~mL})$ was withdrawn and the amylose content determined as described by Hoover and Ratnayake (2004). AML was expressed as the percentage of amylose leached per $100 \mathrm{~g}$ of dry starch. Three replicate samples were used in the determination.

\subsection{Swelling factor (SF)}

The SF of the native and HMT starches when heated to $60-95^{\circ} \mathrm{C}$ in excess water $(5 \mathrm{~mL})$ was determined according to the method of Tester and Morrison (1990). Starch samples (50 mg, db) were weighed into screw cap tubes, $5 \mathrm{~mL}$ deionized water was added, and the tubes were heated in a shaking water bath at the appropriate temperature for $30 \mathrm{~min}$. The tubes were then cooled rapidly to $20^{\circ} \mathrm{C}$ in an ice water bath and $0.5 \mathrm{~mL}$ of Blue Dextran (Pharmacia MW $2 \times 10^{6}, 5$ $\mathrm{mg} / \mathrm{mL}$ ) was added and the contents mixed by inverting the closed tubes several times. The tubes were centrifuged at $1500 \times \mathrm{g}$ for $10 \mathrm{~min}$ and the absorbance of the supernatant was measured at $620 \mathrm{~nm}$. The absorbance of the reference tube that contained no starch was also measured. Three replicate samples were used in the determination. The swelling factor was reported as the ratio of the volume of the swollen starch granules to the volume of the dry starch. 
Calculation of swelling factor (SF) was based on starch weight corrected to moisture content, assuming a density of $1.4 \mathrm{~g} / \mathrm{mL}$. Free or interstitial-plus-supernatant water (FW) is given by:

$$
\mathrm{FW}(\mathrm{mL})=5.5\left(\mathrm{~A}_{\mathrm{R}} / \mathrm{As}_{\mathrm{s}}\right)-0.5
$$

The initial volume of the starch $\left(\mathrm{V}_{\mathrm{O}}\right)$ of weight $\mathrm{W}(\mathrm{mg})$ is

$$
\mathrm{V}_{0}(\mathrm{~mL})=\mathrm{W} / 1400
$$

The volume of absorbed intragranular water $\left(V_{1}\right)$ is thus

$$
\mathrm{V}_{\mathrm{t}}=5.0-\mathrm{FW}
$$

Hence, the volume of the swollen starch granules $\left(V_{2}\right)$ is

$$
\begin{aligned}
& \mathrm{V}_{2}=\mathrm{V}_{0}+\mathrm{V}_{1} \\
& \mathrm{SF}=\mathrm{V} 2 / \mathrm{V} 0 \\
& \text { be expressed by the single equation } \\
& \qquad S F=1+\left\{(7700 / w) \times\left[\left(A_{s}-A_{r}\right) / A_{s}\right]\right\}
\end{aligned}
$$

This can be expressed by the single equation

\subsection{Differential scanning calorimetry (DSC)}

Gelatinization parameters of native and HMT starches were measured using a Mettler Toledo differential scanning calorimeter (DSC1/700/630/GC200) equipped with a thermal analysis data station and data recording software (STAR@ SW 9.20). Deionized water (11 $\mu$ l) was added with a micro syringe to starch $(3.0 \mathrm{mg}$, db) in the DSC pans, which were then sealed, reweighed and allowed to stand overnight at room temperature before DSC analysis. The scanning temperature 
range and the heating rates were $30-110^{\circ} \mathrm{C}$ and $10^{\circ} \mathrm{C} / \mathrm{min}$, respectively. In all measurements, the thermogram was recorded with an empty aluminum pan as a reference. During the scans, the space surrounding the sample chamber was flushed with dry nitrogen to avoid condensation. The transition temperatures reported are the onset (To), peak (Tp) and conclusion (Tc) temperatures. The enthalpy of gelatinization $(\Delta \mathrm{H})$ was estimated by integrating the area between the thermogram and a base line under the peak and was expressed in terms of J/g of dry starch. Three replicates per sample were analyzed.

\subsection{Rapid Visco ${ }^{\text {rM }}$ Analyser (RVA)}

A Rapid Visco ${ }^{\text {TM }}$ Analyzer RVA-4 (Newport Scientific Pty. Ltd., Warriewood, NSW, Australia) was employed to measure the pasting properties of native and HMT starches $(5 \% \mathrm{db}, 25 \mathrm{~g}$ total weight). The samples were equilibrated at $50^{\circ} \mathrm{C}$ for $1 \mathrm{~min}$, heated at $6^{\circ} \mathrm{C} / \mathrm{min}$ to $95^{\circ} \mathrm{C}$, held at $95^{\circ} \mathrm{C}$ for $5 \mathrm{~min}$, cooled at $6^{\circ} \mathrm{C} / \mathrm{min}$ to $50^{\circ} \mathrm{C}$, and held at $50^{\circ} \mathrm{C}$ for $2 \mathrm{~min}$. The speed was $960 \mathrm{rpm}$ for the first $10 \mathrm{~s}$, then $160 \mathrm{rpm}$ for the remainder of the experiment.

\subsection{Enzymatic hydrolysis}

Enzymatic digestibility studies on native and HMT starches were conducted using a crystalline suspension of porcine pancreatic $\alpha$-amylase (PPA) in $2.9 \mathrm{M}$ sodium chloride containing $3 \mathrm{mM}$ calcium chloride, in which the concentration of $\alpha$-amylase was $26 \mathrm{mg}$ protein $/ \mathrm{mL}$ and the specific activity was 1128 units/mg protein. One unit was defined as the $\alpha$-amylase activity

which liberates $1 \mathrm{mg}$ maltose in $3 \mathrm{~min}$ at $20^{\circ} \mathrm{C}$ at $\mathrm{pH} 6.9$. The procedure was essentially that of Knutson et al. (1982) with a slight modification. Starch $(20 \mathrm{mg})$ was added with $5 \mathrm{~mL}$ deionized water and $4 \mathrm{~mL}$ of $0.1 \mathrm{M}$ phosphate buffer $(\mathrm{pH} 6.9$ ) containing $0.006 \mathrm{M} \mathrm{NaCl}$. The slurry was pre- 
warmed for $30 \mathrm{~min}$ at $37^{\circ} \mathrm{C}$ and gently stirred before adding PPA suspension (12 units/mg starch). The samples were analysed after $3,6,12,24,48$, and $72 \mathrm{~h}$ of incubation at $37^{\circ} \mathrm{C}$ in a constant temperature water bath (New Brunswick Scientific, G76D, Edison, NJ, USA). The reaction mixtures were vortexed on a daily basis to resuspend the deposited granules. Samples were taken at specific time intervals and the digestion reaction was terminated by adding $2 \mathrm{~mL}$ of $95 \%$ ethanol. The hydrolysate was recovered for microscopic study by centrifugation $(2000 \times \mathrm{g}$, $5 \mathrm{~min}$ ) of the mixture and aliquots of the supernatant were analysed for soluble carbohydrate (Bruner, 1964). Controls without PPA but subjected to above experimental conditions were run concurrently. The degree of hydrolysis was defined as the reducing sugars generated in the supernatant, expressed as mg maltose equivalents released per $100 \mathrm{mg}$ dry starch. The hydrolysate was washed three times by suspending it in $10 \mathrm{~mL}$ deionized water and recovered by centrifugation $(2000 \times \mathrm{g}, 5 \mathrm{~min})$. The hydrolysates were then dried at $25^{\circ} \mathrm{C}$ for 2 days. The sugar content of the supernatant was determined as follows.

Determination of reducing value by 3, 5-dinitrosalicylic acid (DNS):

3,5-DNS $(2 \mathrm{~mL})$ was taken into a glass tube and placed in an ice-water bath to chill for $5 \mathrm{~min}$. An aliquot of the sample $(1 \mathrm{~mL})$ was pipetted into $2 \mathrm{~mL}$ of the chilled $3,5-\mathrm{DNS}$ and the reaction mixture was then diluted to $4 \mathrm{~mL}$ with deionized water. The contents were mixed by rapid swirling and returned to the ice-water bath until thoroughly chilled and then the contents were heated in a boiling water bath for exactly $5 \mathrm{~min}$ for colour development. After that, the contents were returned to the ice-water bath prior to colour measurement. After chilling, the final volume was adjusted to $8 \mathrm{~mL}$ with deionized water at room temperature. The contents were mixed by rapid swirling before taking the absorbance measurement at $540 \mathrm{~nm}$. If the sample relative 
absorbance exceeded 1.5 at $540 \mathrm{~nm}$, the spectrophotometer was reset to $590 \mathrm{~nm}$ without delay and the relative absorbance was measured at that wavelength. The apparent sugar content of the sample was determined by calculation from the appropriate regression equation of the standard curves.

$$
\text { Enzyme Hydrolysis } \%)=\frac{\text { Reducing sugar }(\text { as maltose }) \times 0.95 \times 100}{\text { Initial starch weight }(g, d b)}
$$

\subsection{Statistical analysis}

Analytical determinations for the samples were done in triplicate and standard deviations were reported. A comparison of the means was ascertained by Tukey's test, to a $5 \%$ level of significance using analysis of the variance (ANOVA). 


\section{CHAPTER 4}

\section{RESULTS AND DISCUSSION}

\subsection{Composition}

Data on the chemical composition of the potato starches are presented in Table 4.1. The purity of the starches was judged on the basis of low nitrogen $(0.13 \%$ [normal potato], $0.14 \%$ [waxy potato]) and low ash $(0.22 \%$ [normal potato], $0.21 \%$ [waxy potato]) levels. There was no significant difference between the starches with respect to their total phosphorus $(0.07-0.08 \%)$, free lipid $(0.02 \%)$ and bound lipid $(0.05-0.08 \%)$ contents (Table 1). However, the starches differed significantly with respect to apparent amylose $(32.3 \%$ [normal potato], 3.4\% [waxy potato]) and total amylose content (33.5\% [normal potato], 3.4\% [waxy potato]). The total phosphorus content, which is mainly in the form of phosphate monoesters (McPherson and Jane, 1999) was in agreement with those reported in the literature (Gunaratne and Hoover, 2002; McPherson and Jane, 1999).

\subsection{Morphology}

Morphological characteristics such as phosphate and amylose distribution, growth rings, surface characteristics and birefringence patterns of native and HMT starches were determined by microscopy.

\subsubsection{Scanning electron microscopy (SEM)}

Scanning electron micrographs of native NP and WP starches are presented in Fig 4.1. The granule surface of normal potato starch appeared to be smooth with no evidence of pores, indentations or fissures. However, in WP starch, pores were present (mainly in the peripheral regions) of some granules, whereas others were devoid of pores (Fig 4.1 [WP]). 
Table 4.1 Chemical composition (\%) of normal and waxy potato starches ${ }^{3}$

\section{Composition (\%)}

Characteristics

$$
\text { Normal potato Waxy potato }
$$

$\begin{array}{lcc}\text { Moisture } & 17.01 \pm 0.04^{p} & 15.20 \pm 0.04^{q} \\ \text { Ash } & 0.22 \pm 0.01^{p} & 0.21 \pm 0.01^{p} \\ \text { Nitrogen } & 0.13 \pm 0.01^{p} & 0.14 \pm 0.03^{p} \\ \text { Phosphorus } & 0.08 \pm 0.01^{p} & 0.07 \pm 0.01^{p}\end{array}$

Amylose content

Apparent $^{\mathrm{b}}$

Total $^{\mathrm{C}}$

Lipid content

$\mathrm{CM}^{\mathrm{d}}$

$\mathrm{PW}^{\mathrm{e}}$
$32.3 \pm 0.2^{\text {P }}$

$33.5 \pm 0.3^{\mathrm{P}}$

$0.02 \pm 0.01^{\mathrm{P}}$

$0.08 \pm 0.03^{p}$
$3.4 \pm 0.5^{9}$

$3.4 \pm 0.3^{q}$

all data reported on a dry basis and represent the means of three determinations. Means of normal and waxy starches with different superscripts within the same row are significantly different $(\mathrm{p}<0.05)$

betermined by iodine binding without removal of free and bound lipids

cDetermined by iodine binding after removal of free and bound lipids

${ }^{4}$ Lipid extracted from the starch by chloroform-methanol (CM) $2: 1\left(\mathrm{v} / \mathrm{v}\right.$ ) at $25^{\circ} \mathrm{C}$ (mainly free lipids)

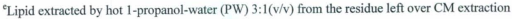
(mainly bound lipids) 
Fig. 4.1 Scanning electron micrographs of native normal (NP) and native waxy (WP) potato starches. 


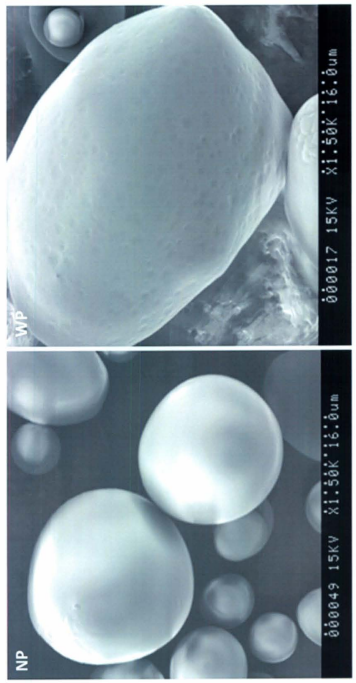




\subsubsection{Confocal laser scanning microscopy (CLSM)}

\subsubsection{Phosphate distribution}

Pro-Q Diamond dye is a metallo-organic fluorophore that recognizes accessible phosphate groups in native starch granules. The distribution of Pro-Q fluorescence reflects phosphate distribution pattern in the granule (Glaring et al., 2006). Pro-Q staining of native NP and WP granules followed by visualization using CLSM revealed that both NP and WP starches were stained brightly near the peripheral regions (Fig 4.2). Smaller granules were stained more brightly (indicating a higher concentration of accessible phosphate groups) than larger granules (Fig 4.2 [NP, WP]). No staining occurred in the granule interior of either starch (Fig 4.2).

Glaring et al. (2006) have shown by Pro-Q staining of cereal and tuber starch granules (representing a wide range in starch bound phosphate content) that fluorescence intensity is positively correlated to phosphate content. The absence of phosphate in the granule interior was surprising (Fig 4.2 [NP, WP]), since Jane and Shen (1993) have shown by surface gelatinization studies that phosphate monoesters are more concentrated at the core (hilum) than in the periphery of normal potato starch granules. Therefore, hypothesized that either the Pro-Q dye is too large (molar mass and structure of this dye is not known) to diffuse into the granule and/or the phosphate groups are buried within the amylopectin clusters and hence not available for interacting with the dye. In order, to test this hypothesis, NP and WP starches were hydrolysed with PPA $(72 \mathrm{~h})$. The residues left after hydrolysis were then stained with Pro-Q Diamond dye. As shown in Fig 4.2 (NP72, WP72), the region near the hilum was stained brightly indicating that PPA hydrolysis may have opened a pathway for the Pro-Q Diamond dye into the amorphous and crystalline regions within the granule interior. 
Fig 4.2 Confocal laser scanning microscopy of Pro-Q Diamond stained granules of native normal potato (NP), native waxy potato (WP), PPA hydrolysed (72 h) normal potato (NP72) and PPA hydrolysed ( 72 h) waxy potato (WP72) starches. 

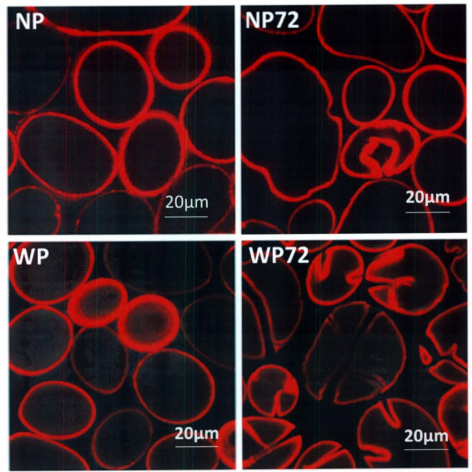


\subsubsection{Growth rings and amylose distribution}

The growth rings and amylose distribution in native and HMT starches stained with APTS was visualized by CLSM. Glaring et al. (2006) have shown that APTS reacts specifically with the reducing ends of starch molecules leading to a 1:1 stoichiometric ratio of starch molecular labelling. Because of its smaller size, amylose (AM) contains a much higher molar ratio of reducing ends per glucose residue than amylopectin (AMP). This results in a higher by-weight labelling of AM enabling the distinction of the two molecules by CLSM.

The CLSM images (Fig 4.3) revealed that both the growth rings and the hilum appeared much sharper and exhibited higher fluorescence in NP than in WP starches. This could be attributed to the higher AM concentration in NP starch. The area near the hilum was disrupted on HMT, resulting in the formation of voids at HMT temperatures of $100^{\circ} \mathrm{C}$ (Fig 4.3 [NP100, WP100]) and $130^{\circ} \mathrm{C}$ (Fig 4.3 [NP130, WP130]). Voids were more evident (WP $>$ NP) at $130^{\circ} \mathrm{C}$ (Fig 4.3 [NP130, WP130]). Buléon et al. (1998) have suggested that surrounding the hilum is a core which is semicrystalline with a lower degree of organization relative to the more crystalline layers at the periphery. The concentration of AM has been shown to increase from the hilum to the periphery (Buléon et al., 1998).

We speculate that the thermal energy imparted during HMT may have disorganized the double helical organization of starch chains present in the core surrounding the hilum region, leading to the formation of voids near the hilum (Fig 4.3). This is reflected in the extent of disorganization in both NP and WP starches being greater at $130^{\circ} \mathrm{C}$ (Fig 4.3 [NP130, WP130]) than at $100^{\circ} \mathrm{C}$ (Fig 4.3 [NP100, WP100]). 
Fig 4.3 Confocal laser scanning microscopy of APTS stained granules of native normal potato (NP), NP heat-moisture treated at $100^{\circ} \mathrm{C}(\mathrm{NP} 100)$, NP heat-moisture treated at $130^{\circ} \mathrm{C}$ (NP130), native waxy potato (WP), WP heat-moisture treated at $100^{\circ} \mathrm{C}$ (WP100) and WP heat-moisture treated at $130^{\circ} \mathrm{C}$ (WP130). 


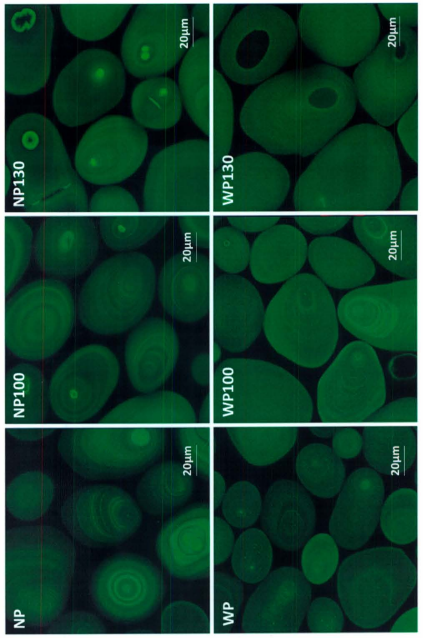




\subsubsection{Polarized and bright field microscopy}

The birefringence patterns of NP and WP starches before and after HMT are shown in Fig 4.4. Birefringence indicates that the average orientation of helical structures is radial. The intensity of birefringence is influenced by granule shape and by the orientation of the granule with respect to the light beam (Buléon et al., 1998), Both NP and WP starches (Fig 4.4) exhibited strong birefringence intensity.

However, the birefringence intensity of both starches decreased with an increase in HMT temperature; the effect was most pronounced in NP130 and WP130 starches (Fig 4.4). The decrease in birefringence intensity was reflected by the formation of voids (depicted by arrows) at the granule centers. This suggests that the increase in starch chain mobility accompanying an increase in HMT temperature disrupted the radial orientation of AMP double helices that were loosely organized around the hilum. Similar observations have been reported for pulse (Chung et al., 2010) and normal potato (Vermeylen et al., 2006) starches. When viewed under bright field microscopy, both HMT NP and WP starches displayed dark areas (voids) near the hilum region in both large and small granules. The size of these voids (representing the extent of disorganization of AMP double helices near the vicinity of the hilum) increased with HMT temperature (Fig 4.4). Furthermore, at $130^{\circ} \mathrm{C}$, voids in WP starch were larger and more numerous than in NP130 starch. 
Fig 4.4 Polarized light microscopy of native and HMT normal potato (NP) and waxy potato (WP) starches. For NP and WP starches, the same field is shown for bright field (left frame) and between cross polarized light (right frame). The arrows depict the regions in the granules that were disorganized during HMT. 


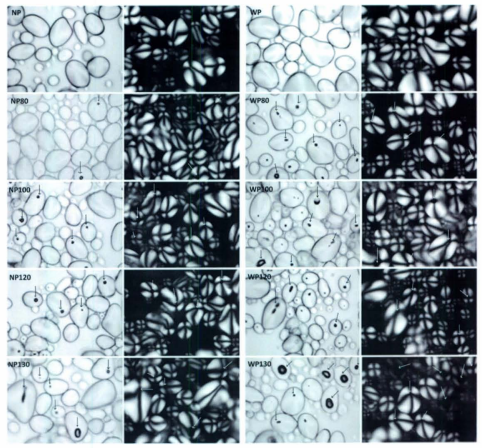




\subsection{Amylopectin chain length distribution}

The amylopectin chain length distributions (determined by fluorophore assisted capillary electrophoresis) of both native and HMT starches remained unchanged at all HMT temperatures (Table 4.2). This suggests that covalent linkages of AMP were not broken under the HMT conditions $\left(27 \%\right.$ moisture, $\left.16 \mathrm{~h}, 80-130^{\circ} \mathrm{C}\right)$ used in this study. However, Vermeylen et al., (2006) reported degradation of amylopectin crystallites (indicated by an increase in the proportion of chains with $\mathrm{DP}<6$ ) during HMT of NP starch at $23 \%$ moisture for $24 \mathrm{~h}$ at $130^{\circ} \mathrm{C}$. This discrepancy may have been due to the longer period of HMT used in their study.

\subsection{X-ray diffraction}

NP (Fig 4.5a) and WP (Fig 4.5b) starches exhibited the typical B-type X-ray pattern with reflection intensities centred at $5.5^{\circ}, 17.1^{\circ}$ and $22-24^{\circ} 2 \theta$. The peak height $(\mathrm{PH})$ and the peak width at half height, also called full width at half maximum height (FWHM) [Table 4.3] of the $5.5^{\circ} 2 \theta$ peak (typical of B-type crystallites; Hizukuri et al,, 1983) for NP and WP starches were 178.6 and 0.9 and 106.6 and 1.0 , respectively. PH and FWHM have been shown to represent the amount of ordered crystallites and crystallite size and/or crystallite perfection, respectively (Suryanarayana and Norton, 1998). This suggests a higher content of ordered B-type crystallites in NP starch, which are larger in size than those of WP starch. In WP starch, the doublet centred at $22-24^{\circ} 2 \theta$ was less well resolved (Fig 4.5b) than in NP starch (Fig 4.5a). This is indicative of the presence of both A- and B-type unit cells in WP starch. Both NP and WP starches exhibited a peak centred at $20^{\circ} 2 \theta$, which reflects single left handed linear starch chains arranged in a Vtype crystalline array (Lopez-Rubio et al., 2008a; Saibene et al., 2008). The PH and FWHM of the $20^{\circ} 2 \theta$ peak for NP and WP starches were 51 and 0.6 and 47.7 and 0.9 , respectively. 
Table 4.2 Amylopectin chain length distribution of native and HMT normal and waxy potato starches as determined by fluorophore-assisted capillary electrophoresis (FACE)

\begin{tabular}{lccccc}
\hline Starch source & Treatment & \multicolumn{4}{c}{ Distribution percentage } \\
\cline { 3 - 6 } & & DP 6-8 & DP 9-13 & DP 14-35 & DP 36-49 \\
\hline Normal potato & Native & $7.95 \pm 0.27^{p}$ & $29.37 \pm 1.39^{p}$ & $59.50 \pm 1.59^{p}$ & $3.18 \pm 0.47^{p}$ \\
& NP80 & $6.84 \pm 0.54^{p}$ & $27.27 \pm 1.09^{p}$ & $61.49 \pm 0.33^{p}$ & $4.40 \pm 0.22^{p}$ \\
& NP100 & $7.11 \pm 1.11^{p}$ & $26.01 \pm 2.97^{p}$ & $61.56 \pm 1.39^{p}$ & $5.32 \pm 2.70^{p}$ \\
NP120 & $8.38 \pm 0.02^{p}$ & $28.04 \pm 0.06^{p}$ & $58.88 \pm 0.36^{p}$ & $4.70 \pm 0.45^{p}$ \\
Waxy potato & N130 & $8.63 \pm 0.24^{p}$ & $27.64 \pm 0.82^{p}$ & $58.76 \pm 0.76^{p}$ & $4.97 \pm 0.29^{p}$ \\
& Native & $6.43 \pm 0.95^{p}$ & $26.83 \pm 1.01^{p}$ & $60.37 \pm 0.72^{p}$ & $6.09 \pm 0.45^{p}$ \\
& WP80 & $7.51 \pm 0.42^{p}$ & $29.00 \pm 0.60^{p}$ & $58.96 \pm 0.10^{p}$ & $4.53 \pm 1.13^{p}$ \\
& WP100 & $7.14 \pm 0.65^{p}$ & $27.37 \pm 1.94^{p}$ & $60.00 \pm 1.04^{p}$ & $5.49 \pm 1.56^{p}$ \\
& WP120 & $6.76 \pm 0.72^{p}$ & $29.78 \pm 3.23^{p}$ & $58.35 \pm 3.94^{p}$ & $5.11 \pm 0.01^{p}$ \\
& WP130 & $6.43 \pm 0.95^{p}$ & $25.87 \pm 0.43^{p}$ & $61.97 \pm 1.04^{p}$ & $5.73 \pm 0.34^{p}$ \\
\hline
\end{tabular}

Means within the same column with different superscripts for native and its HMT counterparts are significantly different ( $p<0.05)$ 
This suggests a higher content of ordered V-type crystallites in NP starch, which are larger in size than those of WP starch. It is highly unlikely that the V-type crystallites are indicative of starch-lipid complexes, since both starches contained only trace quantities of bound lipids $(<0.08 \%)$. HMT, at all temperatures, changed the $\mathrm{X}$-ray pattern $(\mathrm{B} \rightarrow \mathrm{A}+\mathrm{B})$ and decreased the relative crystallinity (RC) and B-polymorphic content (Fig 4.5). The extent of these changes increased progressively with an increase in HMT temperature. The change in X-ray pattern has been attributed to dehydration of the 36 water molecules in the central channel of the B-type unit cell and to the movement of a pair of double helices into the central channel that was originally occupied by the water molecules (Gunaratne and Hoover, 2002). This would explain the increase in A-type unit cells with an increase in HMT temperatures. The increase in A-type unit cells was reflected in the decrease in PH of the reflections centred at 5.6 $2 \theta$ (NP>WP), $17.12 \theta$ $(\mathrm{NP}>\mathrm{WP})$, merging of the doublet centred at $22-24^{\circ} 2 \theta(\mathrm{WP}>\mathrm{NP})$ and broadening of the $17.1^{\circ} 2 \theta$ peak (WP> NP) [Fig 4.5, Table 4.3]. The increase in peak width of the reflection centred at $5.5^{\circ}$ $2 \theta$ in NP and WP starches on HMT (Fig 4.5, Table 4.3) suggests a reduction in the size of B-type crystallites and/or dissociation of these crystallites within the crystalline lattice during the polymorphic transformation. HMT at $80^{\circ} \mathrm{C}$ decreased the $\mathrm{PH}$ of the reflection centred at $20^{\circ} 2 \theta$ (NP80 $>$ WP80) (Table 4.3 Fig 4.5). The size of the crystallites (Table 4.3, Fig 4.5) forming this peak remained unchanged in NP80 starch, but decreased in WP80 starch. The decrease in PH at $80^{\circ} \mathrm{C}$ indicates that the ordered crystallites of the V-type helices (representing single AM chains and/or the outer branches of AMP) may have been disrupted due to an increase in chain mobility. The $20^{\circ} 2 \theta$ reflection was not visible in NP100, NP120, NP130, WP100, WP120 and WP130 starches (Fig 4.5). 
Fig 4.5 X-ray diffraction patterns of NP (a), WP (b), NP80 (c), WP80 (d), NP100 (e), WP100 (f), NP120 (g), WP120 (h), NP130 (i) and WP130 (j) starches. 

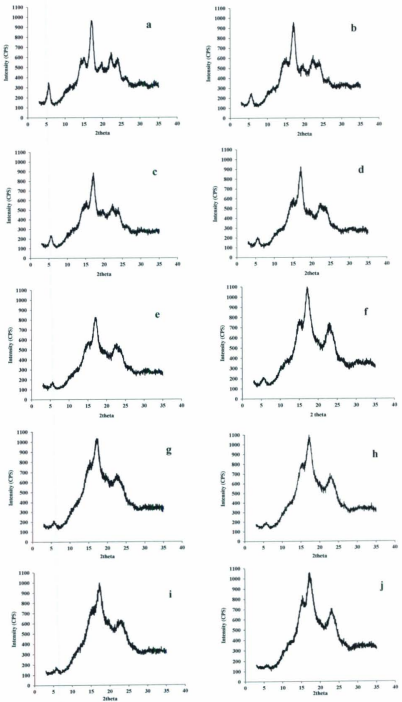


\subsection{ATR-FTIR spectroscopy}

The ATR-FTIR spectral data for native and HMT starches are presented in Table 4.4. This technique is considered as a short-range probe, measuring order at the level of individual helices. That is, it distinguishes helices either in or out of the crystalline registry, unlike X-ray diffraction, which is related to the packing of double helices into ordered crystalline arrays. In the spectrum of native potato starch obtained by the FTIR method, the following bands can be distinguished (Cael et al., 1973, 1975; van Soest et al., 1995): 2,900-3,000 $\mathrm{cm}^{-1}$ (stretching vibration of $\mathrm{CH}$ ), 1,150,1,124 and $1,103 \mathrm{~cm}^{-1}$ (stretching vibrations of $\mathrm{CO}, \mathrm{CC}$ and $\mathrm{COH}$ ), 1,047, 1,022, 994 and $928 \mathrm{~cm}^{-1}$ (bending vibrations of $\mathrm{COH}$ and $\mathrm{CH}_{2}$ ), $861 \mathrm{~cm}^{-1}$ (symmetric stretching vibrations of $\mathrm{COC}$ and deformation vibrations of $\mathrm{CH}$ ). Sevenou et al. (2002) have shown that ATR-FTIR yields information on the structural organization of starch chains near the granule surface, since the IR beam penetrates only to a depth of $2 \mu \mathrm{m}$ into the granule. The above authors showed that FTIR differences between starch varieties are related only to variations in the ratios of the amounts of ordered to unordered fractions within the starch granule.

The FTIR spectrum of starch has been attributed to three main vibrational modes with maximum absorbances at 995,1022 and $1047 \mathrm{~cm}^{-1}$. The IR bands at $1048 \mathrm{~cm}^{-1}$ and $1016 \mathrm{~cm}^{-1}$ have been shown to be associated with ordered and amorphous structures of starch, respectively (van Soest et al., 1995). Studies on normal potato starch have shown that the band at $1048 \mathrm{~cm}^{-1}$ increases

with an increase in crystallinity (van Soest et al., 1995). The band at $1016 \mathrm{~cm}^{-1}$ has been attributed to vibrational modes within the amorphous domains of starch granules [since this band has been observed to decrease with an increase in crystallinity (Capron et al, 2007)]. 
Table 4.4 Short range molecular order of native and heat-moisture treated potato starches measured by attenuated total reflectance-Fourier transform infrared spectroscopy (ATR-FTIR)

\begin{tabular}{ccc}
\hline Starch source & Treatment & Ratio $\left(1048 / 1016 \mathrm{~cm}^{-1}\right)^{\mathrm{a}}$ \\
\hline Normal potato & Native & $1.146 \pm 0.011^{\mathrm{p}}$ \\
& HMT 80 & $1.078 \pm 0.002^{\mathrm{q}}$ \\
& HMT 100 & $1.000 \pm 0.000^{\mathrm{f}}$ \\
& HMT 120 & $0.889 \pm 0.000^{\mathrm{s}}$ \\
& HMT 130 & $0.886 \pm 0.005^{\mathrm{s}}$ \\
Waxy potato & Native & \\
& HMT 80 & $1.167 \pm 0.011^{\mathrm{p}}$ \\
& HMT 100 & $1.095 \pm 0.000^{\mathrm{q}}$ \\
& HMT 120 & $1.000 \pm 0.000^{\mathrm{t}}$ \\
& HMT 130 & $0.917 \pm 0.039^{\mathrm{f}}$ \\
& & $0.927 \pm 0.032^{\mathrm{f}}$ \\
\hline
\end{tabular}

"Ratio of the ordered crystalline $\left(1048 \mathrm{~cm}^{-1}\right)$ to amorphous domains $\left(1016 \mathrm{~cm}^{-1}\right)$.

Means within each column with different superscripts for the native potato starch and its heatmoisture treated counterparts are significantly different $(\mathrm{P}<0.05)$ 
Thus, the ratio of the bands at $1048 \mathrm{~cm}^{-1}$ and $1016 \mathrm{~cm}^{-1}$ reflects the proportions of ordered starch and amorphous starch. The band at $995 \mathrm{~cm}^{-1}$ which is mainly due to $\mathrm{COH}$ bending vibrations, is especially sensitive to water content and these vibrations involve water-starch interaction, such as hydrogen bonding, which influence the $\mathrm{COH}$ bending modes.

HMT at all temperatures decreased the IR ratio $\left(1048 \mathrm{~cm}^{-1} / 1016 \mathrm{~cm}^{-1}\right)$ in both NP and WP starches by nearly the same amount, the extent of this reduction being more pronounced at $120^{\circ}$ and $130^{\circ} \mathrm{C}$ (Table 4.4). There was no significant difference in the IR ratio $\left(1048 \mathrm{~cm}^{-1} / 1016 \mathrm{~cm}^{-1}\right.$ ) between NP120 and NP130 starches. The progressive decrease in the IR ratio $\left(1048 \mathrm{~cm}^{-1} / 1016\right.$ $\mathrm{cm}^{-1}$ ) with an increase in HMT temperature reflects the interplay between: 1) the extent of polymorphic transformation $(\mathrm{B} \rightarrow \mathrm{A}+\mathrm{B}), 2$ ) disruption of hydrogen bonds, and 3) disorganization of double helices within AMP clusters.

\subsection{Amylose leaching (AML)}

The extent of AML in the temperature range of $60-95^{\circ} \mathrm{C}$ is presented in Fig 4.6. In both native and HMT starches, AML increased with increase in temperature. HMT of NP starch at $80^{\circ}$ and $100^{\circ} \mathrm{C}$ decreased $\mathrm{AML}$. The extent of this decrease followed the order: NP80 $>$ NP100 $>$ NP (Fig 4.6). $\mathrm{AML}$ in NP80 starch was detectable only at temperatures exceeding $75^{\circ} \mathrm{C}$. NP120 and NP130 starches exhibited higher AML than NP starch in the temperature range $60-75^{\circ} \mathrm{C}$, but lower AML than NP starch in the temperature range $75^{\circ}-95^{\circ} \mathrm{C}$ (Fig 4.6). There was no significant difference in the extent of AML between NP120 and NP130 starches in the temperature range $60-95^{\circ} \mathrm{C}$. 
Fig 4.6 Amylose leaching of native and heat-moisture treated normal potato (NP) starches at 60$95^{\circ} \mathrm{C}$. 


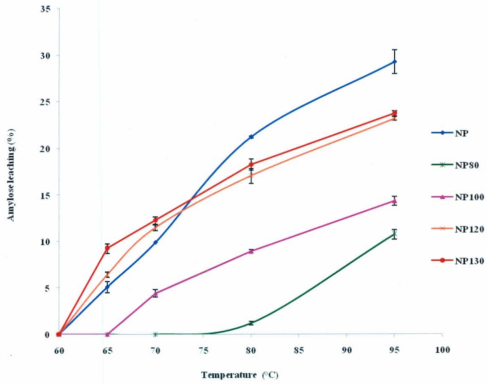


The reduction in AML on HMT has been attributed to the interplay of: 1) additional interactions between AM-AM and/or AM-AMP chains, 2) a decrease in granular swelling and 3) an increase in V-amylose lipid content (Hoover and Vasanthan, 1994b). In this study, only factors 1 and 2 influence AML, since lipids were present only in trace $(<0.08 \%)$ quantities (Table 4.1$)$ in NP starch. The results showed that AM-AM and/or AM-AMP interactions were most extensive in NP80 starch. This indicates that due to the lower degree of chain mobility at $80^{\circ} \mathrm{C}$, starch chains are better aligned for interaction. The order of AML in the temperature range $60-75^{\circ} \mathrm{C}$ (NP120 NP130>NP) indicates that at $120^{\circ} \mathrm{C}$ and $130^{\circ} \mathrm{C}$, hydrogen bonds between cocrystallized AM and AMP chains may have been disrupted. However, the extent of AML in NP120 and NP130 starches was lower than in NP starch (NP>NP120 NP130) in the temperature range $75-95^{\circ} \mathrm{C}$. This could be attributed to lower granular swelling in NP120 and NP130 starches (Fig 4.7). A decrease in granular swelling would reduce AML.

\subsection{Swelling factor (SF)}

The swelling factor in the temperature range $60-95^{\circ} \mathrm{C}$ of NP and WP starches before and after HMT are presented in Figs 4.7. The SF of native NP starch (Fig 4.7a) increased progressively with an increase in temperature. The extent of this increase was most pronounced in the temperature range $60-70^{\circ} \mathrm{C}$. However, in WP starch, SF increased only until $70^{\circ} \mathrm{C}$, and then decreased thereafter (Fig 4.7b). The SF of starches has been shown to be influenced by amylopectin content and structure and starch-lipid complexes (Gomand et al., 2010; Chung et al., 2009a). Since there was no difference in chain length distribution of amylopectin (Table 4.2) or bound lipid content (Table 4.1) between the starches, the higher SF of waxy potato in the temperature range $60-70^{\circ} \mathrm{C}$ reflects its higher amylopectin content. The lower SF of waxy potato 
starch at higher temperatures $\left(>70^{\circ} \mathrm{C}\right)$ suggests that the higher granular swelling at lower temperatures rendered the granules very fragile and thus susceptible to partial disintegration at higher temperatures.

HMT reduced the SF of NP starches at all temperatures (Fig 4.7a). Reduction in SF on HMT has also been reported in cereals, pulses and tuber starches (Chung et al., 2009; Hoover et al., 2010; Jayakody et al., 2007). The extent of SF reduction (in the temperature range $70-95^{\circ} \mathrm{C}$ ) followed the order: NP100 NP130 $>$ NP120 $>$ NP80. The reduction in SF on HMT has been attributed to : 1) AM-AM, AM-AMP and AMP-AMP interactions (these interactions reduces the number of free hydroxyl groups available for interaction with water) and 2) polymorphic transformation $(B \rightarrow A+B)$ [the ingress of water during SF measurements would be more rapid and higher in the B-type polymorph (Hoover and Vasanthan, 1994b) due to double helices being more compactly packed in the A-type polymorph]. X-ray data (Fig 4.5) showed that the amount of A-type polymorph in the HMT starches followed the order: NP130>NP120>NP100>NP80. In order to explain the variation in SF reduction on HMT, it is necessary to consider both AML (Fig 4.6) and DSC (Table 4.5) data. AML data showed that starch chain interactions (AM-AMP, AMPAMP) followed the order: NP8 $0>\mathrm{NP} 100>\mathrm{NP} 120>\mathrm{NP} 130$. The large decrease in $\Delta \mathrm{H}$ at $130^{\circ} \mathrm{C}$ showed disorganization of crystallite packing. Thus, the differences in SF among the HMT NP starches (Fig4.7a) reflect the interplay between the following factors: 1) extent of interaction among AM-AMP and AMP-AMP chains, 2) the amount of A-type unit cells, 3) crystalline stability and 4) the extent of organization of double helical packing within the amylopectin clusters. 
Fig 4.7 Swelling factor in the temperature range $60-90^{\circ} \mathrm{C}$ of native and heat-moisture treated normal potato (NP) (a) and waxy potato (WP) (b) potato starches. 


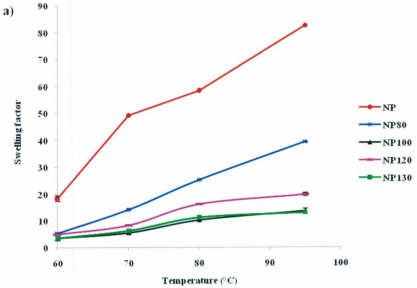

b)

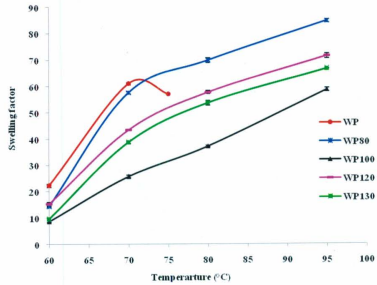


In the WP starches (Fig4.7b), SF of WP starch increased up to $70^{\circ} \mathrm{C}$ and then decreased due to the disruption of highly swollen granules. The rapid increase in SF in the low temperature range reflects the higher AMP content of WP starch. The difference in SF between WP80 and WP100 starches reflects the higher content of A-type unit cells in the latter (Fig 4.5). The differences in SF between WP120 and WP130 starches reflect not only the presence of more A-type unit cells but also the greater loss in crystallite packing (Table 4.5) in the latter.

\section{$4.8 \mathrm{~K} / \mathrm{S}$ spectral analysis}

The K/S spectra provide information about the intensity of the colour developed in granular starch when it complexes with iodine, forming V-type crystallinity (Saibene and Seetharaman, 2010; Saibene et al., 2006). Saibene et al. (2006) and Waduge et al. (2010) have shown that iodine binding in a starch granule requires a certain minimum level of mobility of the linear molecules, and that the water content would plasticize the starch, thus increasing the ability to bind iodine by the formation of single helices.

Gidley and Bulpin (1987) have shown that all $\alpha$-1,4-glucans form helical inclusion complexes with iodine to some degree. The extent of the iodine binding depends upon the degree of helix formation which can be attained. A single helix, capable of supporting complex formation, has been shown to require six glucose molecules per turn. As the length of the glucan chain, and hence the number of helical turns, increases, the number of iodine molecules which can be accommodated aiso increases, so that the iodine binding capacity is enlarged. This increased binding has been shown to result in a shift in the wavelength of maximum absorbance; $1 / \lambda_{\max }$ has been shown to be directly proportional to 1/DP up to a DP of about 100 (Banks et al., 1971) The 
formation of inclusion complexes between granular starches and iodine vapour has been shown to depend on the accessibility of segments of glucan polymers (segments of AM molecules which are not trapped in the crystalline lamellae, AM molecules that are not in double helices with AMP branch chains and long segments of AMP branches) to iodine (Waduge and Seetharaman, 2010). Therefore, the structural organization of starch chains within the amorphous and crystalline domains will influence the extent of iodine binding. The major structural changes during HMT at different temperatures and the availability of glucan polymers for iodine complexing before and after HMT are presented schematically in Fig 4.8.

The K/S spectra of NP and WP starches before and after HMT $\left(80,100,120,130^{\circ} \mathrm{C}\right)$ following equilibration at $100 \%$ relative humidity are presented in Fig 4.9. NP and NP120 starches exhibited maximum K/S absorption of 39.0 and 42.0, respectively, at $590 \mathrm{~nm}$. However, NP80, NP100 and NP130 starches exhibited K/S maxima of 38.2, 31.0 and 37.5, respectively, at 570 $\mathrm{nm}$. The decrease in K/S maxima and $\lambda_{\max }(590$ to $570 \mathrm{~nm}$ ) shown by NP80 starch indicates that AM-AM and/or AM-AMP interactions during HMT decrease the population of glucan polymers (decreases the $\mathrm{K} / \mathrm{S}$ maxima) and chain length (decreases $\lambda_{\max }$ ) available for iodine complexing. AML data (Fig 4.6) showed that interactions between AM-AM and AM-AMP chains were most pronounced in NP80 starch. Consequently, these interactions would decrease chain mobility, thereby hindering the ability of AM chains to form helical complexes with iodine. AML data (Fig 4.6) also showed that NP100 starch exhibited a higher degree of AML than NP80 starch. Theoretically, NP100 starch should have exhibited a higher K/S maxima than NP80 starch due to the availability of additional free AM chains for iodine complexing. 
Fig 4.8 Schematic model for structural changes on HMT at different temperatures (A) and their impact on iodine binding (B).

A] Arrangement of amylose and amylopectin chains in the native starch granule (a) and after HMT at $80-100^{\circ} \mathrm{C}(\mathrm{b}), 120^{\circ} \mathrm{C}(\mathrm{c}), 130^{\circ} \mathrm{C}$ (d)

B] Iodine complexing ability of glucan polymers before (a) and after HMT at 80 $100^{\circ} \mathrm{C}$ (b), $120^{\circ} \mathrm{C}$ (c), $130^{\circ} \mathrm{C}$ (d)

At low temperatures $\left(80-100^{\circ} \mathrm{C}\right)$ - AM-AM, AM-AMP and AMP-AMP interactions reduce the availability of glucan polymers for iodine complexing

At $120^{\circ} \mathrm{C}$ - disruption of hydrogen bonds between co-crystallized AM and AMP chains and between AMP-AMP chains increases the availability of glucan polymers for iodine complexing

At $\left.130^{\circ} \mathrm{C}-1\right)$ disruption of hydrogen bonds between co-crystallized AM and AMP chains and between AMP-AMP chains increases the availability of glucan polymers for iodine complexing 2) formation of interrupted helices reduces the availability of glucan polymers for iodine complexing 

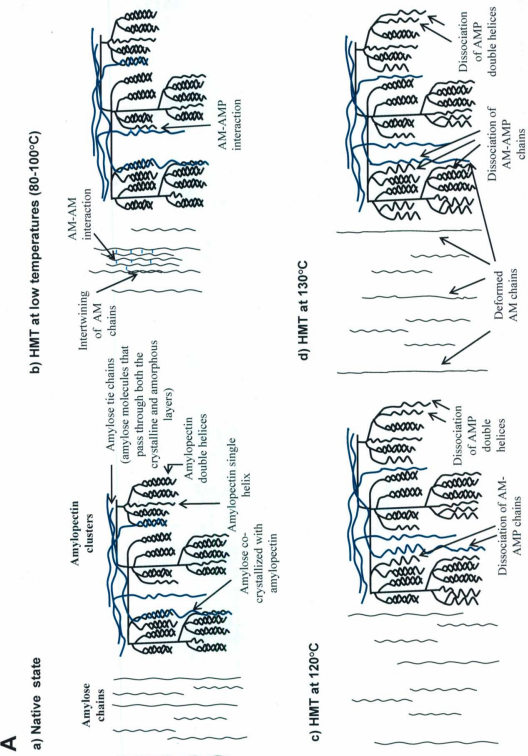

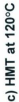

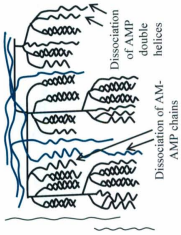



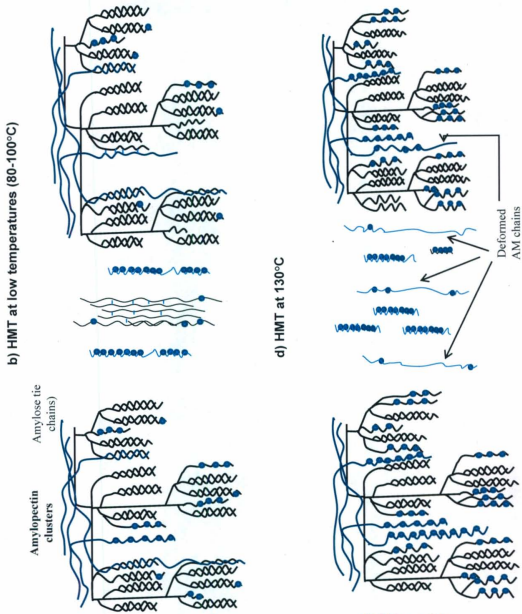

vepesee verea.

verpeceer reape.

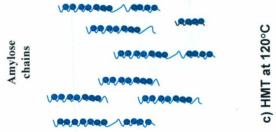

ํㅜㅇ vopeseen vere vepace vera vepeesen

vesaeen veresen peresea reses. 
However, as shown in Fig 4.9a, the iodine complexing ability of NP100 starch was much lower than that of NP80 starch. As discussed earlier, the polymorphic transformation during HMT is accompanied by the loss of water from the central channel of the B-type unit cell (Gunaratne and Hoover, 2002). Consequently, the extent of starch chain plasticization would decrease with an increase in HMT temperature. This in turn would decrease iodine binding ability. Thus, the difference in K/S maxima between NP80 and NP100 starches, and the decrease in $\lambda_{\max }$ at HMT temperatures of $80^{\circ}$ and $100^{\circ} \mathrm{C}$ stands explained. The difference in $\mathrm{K} / \mathrm{S}$ maxima (Fig 4.9a) between NP120 (42.0) and NP (39.0) starches at the same $\lambda_{\max }(590 \mathrm{~nm})$ is indicative of the availability of a greater population of glucan polymers for iodine complexing in NP120 starch. This seems to suggest that the iodine binding ability of NP120 starch is not influenced by the lower degree of plasticization of its starch chains. DSC data (Table 4.5) showed that AM-AMP interactions were disrupted at $120^{\circ} \mathrm{C}$ and $130^{\circ} \mathrm{C}$. These disruptions would free $\mathrm{AM}$ and AMP chains, thereby increasing their mobility and hence their ability to bind iodine. This would then account for the difference in K/S maxima between NP120 and NP100 starches. On this basis, one would have expected the K/S maximum of NP130 starch to be higher than that of NP120 starch. However, the lower K/S maxima of NP130 starch and the shift to a lower $\lambda_{\max }(570 \mathrm{~nm})$ at $130^{\circ} \mathrm{C}$, suggests that intra molecular hydrogen bonds stabilizing the helical region may have been disrupted, resulting in interrupted single helices with limited regions of random coil interspaced between helical segments. A helix to coil transformation would decrease the number of helical turns required to form the typicai deep blue colour with iodine. It is highly unlikely that a coil $\rightarrow$ helix transition could occur on the addition of iodine vapour to NP130 starch, since an aqueous medium would be required for such a transformation (Cheetham and Tao, 1998). 
Fig 4.9 Reflectance spectra of native and heat-moisture treated normal potato (NP) (a) and waxy potato (WP) (b) starches. 


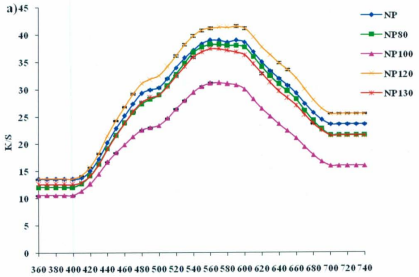

Wavelength (nm)

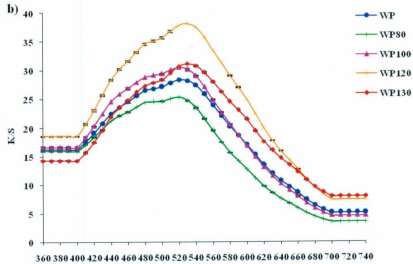

Wavelength (am) 
WP and WP 80 starches exhibited a K/S maxima of 28 and 25 , respectively, at $520 \mathrm{~nm}$. However, HMT at 100,120 and $130^{\circ} \mathrm{C}$ increased the K/S maximum (WP100 [30 at $520 \mathrm{~nm}$ ], WP120 [38 at $530 \mathrm{~nm}$ ] and WP130 [31 at $530 \mathrm{~nm}]$ ). The decrease in $\mathrm{K} / \mathrm{S}$ maxima at $80^{\circ} \mathrm{C}$ is indicative of interactions between AMP-AMP chains, Disruption of hydrogen bonds between AMP-AMP chains at higher temperatures of HMT $\left(>80^{\circ} \mathrm{C}\right)$ may have rendered the outer branches of AMP more flexible for complexing iodine. The difference in K/S maxima between WP120 and WP130 starches is indicative of the presence of a higher content of interrupted helices in the latter. It was interesting to observe that the K/S maximum of NP100 starch was lower than that of NP starch (Fig 4.9a), whereas that of WP100 starch was greater than that of WP starch (Fig 4.9b). This could be attributed to strong interactions between AM-AM and/or AM-AMP chains during $\mathrm{HMT}$ of $\mathrm{NP}$ starch at $100^{\circ} \mathrm{C}$, and to decreased starch chain plasticization at this temperature. The presence of only trace quantities of AM in WP starch may have facilitated the disruption of hydrogen bonds between AMP chains during HMT at $100^{\circ} \mathrm{C}$, thereby increasing the ability of WP100 starch to bind more iodine than WP starch.

\subsection{Gelatinization characteristics}

The gelatinization temperatures ([To-onset], [Tp-midpoint], [Tc-conclusion]), the gelatinization temperature range (Tc-To) and the enthalpy of gelatinization $(\Delta \mathrm{H})$ of normal potato $(\mathrm{NP})$ and waxy potato (WP) starches, before and after HMT, are presented in Table 4.5. To, Tp and Te are influenced by the structural organization of the AMP clusters. $\Delta \mathrm{H}$ has been suggested to reflect the following: 1) melting of double helices (Cooke and Gidley, 1992), 2) overall crystallinity (quantity and amount of AMP crystallites) (Tester and Morrison, 1990) and 3) melting of imperfect amylopectin-based crystals with potential contributions from both crystal packing and 
helix melting enthalpies (Lopez-Rubio et al.,2008b). HMT of NP and WP starches at 80 and $100^{\circ} \mathrm{C}$ increased $\mathrm{Tp}$ and $\mathrm{Tc}$. The extent of this increase being higher at $100^{\circ} \mathrm{C}$ (Table 4.5 ). However, this trend was reversed at 120 and $130^{\circ} \mathrm{C}$, where the extent of increase in $\mathrm{Tp}$ and $\mathrm{Tc}$ was lower than at $100^{\circ} \mathrm{C}$. An increase in Tp and Te on HMT of tuber starches has been shown to represent melting of : a) B-type crystallites present in the native granule, b) A-type crystallites formed during the polymorphic transformation, and c) crystallites formed from interaction between AM-AMP and AMP-AMP chains (Hoover, 2010).

The increase in A-type crystallites on HMT in both NP and WP starches (Fig 4.5), when considered along with compact packing of double helices in A-type crystallites, should have theoretically resulted in Tp and Tc of NP120, NP130, WP120 and WP130 starches being higher than their respective counterparts at $100^{\circ} \mathrm{C}(\mathrm{NP100}$, WP100). This suggests that the higher chain mobility prevailing at $120^{\circ}$ and $130^{\circ} \mathrm{C}$ may have hindered the expected increase in $\mathrm{Tp}$ and $\mathrm{Tc}$ by decreasing the extent of interaction between AM-AMP and AMP-AMP chains and/or by disrupting hydrogen bonds between co-crystallized AM and AMP chains (decreases crystalline stability).

HMT decreased $\Delta H$ in both NP and WP starches (Table 4.5). The extent of this decrease for NP and WP starches followed the order: NP130 $>$ NP120-NP100 $>$ NP80 and WP130 $>$ WP120 $>$ WP100 $>$ WP80, respectively. The decrease in $\triangle H$ for NP80, NP100, WP80 and WP100 starches can be attributed to interactions between AM-AMP and/or AMP-AMP chains on HMT that strengthen double helical associations (Fig 4.6). 
Table 4.5 Gelatinization parameters of native and HMT normal and waxy potato starches

\begin{tabular}{|c|c|c|c|c|c|c|}
\hline \multirow[t]{2}{*}{ Starch source } & \multirow[t]{2}{*}{ Treatment } & \multicolumn{5}{|c|}{ Gelatinization transition parameters ${ }^{\mathrm{a}}$} \\
\hline & & To $\left({ }^{\circ} \mathrm{C}\right)^{b}$ & $\operatorname{Tp}\left({ }^{\circ} \mathrm{C}\right)^{b}$ & $\operatorname{Tc}\left({ }^{\circ} \mathrm{C}\right)^{b}$ & Te- To $\left({ }^{\circ} \mathrm{C}\right)^{c}$ & $\Delta \mathrm{H}(\mathrm{J} / \mathrm{g})^{\mathrm{d}}$ \\
\hline \multirow[t]{5}{*}{ Normal potato } & Native & $62.08 \pm 0.3^{p}$ & $66.92 \pm 0.1^{p}$ & $72.59 \pm 0.0^{\mathrm{p}}$ & $10.51 \pm 0.3^{p}$ & $13.20 \pm 0.2^{p}$ \\
\hline & NP80 & $64.32 \pm 0.2^{q}$ & $70.09 \pm 0.1^{9}$ & $76.06 \pm 0.6^{9}$ & $11.75 \pm 0.4^{p}$ & $10.73 \pm 0.3^{q}$ \\
\hline & NP100 & $63.87 \pm 0.3^{9}$ & $72.98 \pm 0.1^{r}$ & $80.39 \pm 0.2^{r}$ & $16.52 \pm 0.2^{4}$ & $9.05 \pm 0.4^{r}$ \\
\hline & NP120 & $61.72 \pm 0.3^{p}$ & $67.89 \pm 0.2^{p}$ & $75.33 \pm 0.4^{\mathrm{q}}$ & $13.61 \pm 0.1^{r}$ & $9.02 \pm 0.6^{r}$ \\
\hline & NP130 & $60.65 \pm 0.2^{r}$ & $67.14=0.5^{p}$ & $76.54 \pm 0.9^{\mathrm{q}}$ & $15.89 \pm 0.6^{\mathrm{q}}$ & $7.36 \pm 0.4^{5}$ \\
\hline \multirow[t]{5}{*}{ Waxy potato } & Native & $63.21 \pm 0.1^{\mathrm{P}}$ & $68.38 \pm 0.0^{p}$ & $73.85 \pm 0.3^{p}$ & $10.64 \pm 0.4^{p}$ & $14.50 \pm 0.2^{P}$ \\
\hline & WP80 & $63.15 \pm 0.2^{p}$ & $69.33 \pm 0.0^{4}$ & $75,41 \pm 0.1^{p, r}$ & $12.26 \pm 0.1^{9}$ & $12.24=1.0^{9}$ \\
\hline & WP100 & $61.67 \pm 0.1^{4}$ & $74.63 \pm 0.1^{t}$ & $81.54=0.1^{5}$ & $19.88 \pm 0.0^{r}$ & $11.86 \pm 0.6^{q}$ \\
\hline & WP120 & $61.30 \pm 0.1^{q}$ & $67.70 \pm 0.0^{5}$ & $75.89 \pm 0.3^{r}$ & $14.58 \pm 0.4^{5}$ & $10.01 \pm 0.2^{r}$ \\
\hline & WP130 & $61.65 \pm 0.1^{q}$ & $68.32 \pm 0.1^{P}$ & $79.98 \pm 0.8^{t}$ & $18.33 \pm 0.7^{t}$ & $8.46 \pm 0.3^{5}$ \\
\hline
\end{tabular}

"All data reported on dry basis. Means within each column with different superseripts for native and waxy potato starches and their heat moisture treated counterparts are significantly different $(\mathrm{P}<0.05)$ by Tukey's HSD test

${ }^{b} \mathrm{To}, \mathrm{Tp}, \mathrm{Tc}$ represent the onset, mid-point and conclusion temperature respectively

' $(\mathrm{Tc}-\mathrm{To})$ represents the gelatinization temperature range

${ }^{d}$ Gelatinization enthalpy of starch expressed in J/g of dry starch 
Consequently, the number of helices that disrupt, unwind and melt during gelatinization would decrease on HMT. Tp and Tc data (Table 4.5) revealed that starch chain interactions at HMT temperatures of $120^{\circ}$ and $130^{\circ} \mathrm{C}$ were weaker than at $100^{\circ} \mathrm{C}$ (Table 4.5 ). This suggests that the decrease in $\Delta \mathrm{H}$ at $120^{\circ}$ and $130^{\circ} \mathrm{C}$ reflect mainly disruption of hydrogen bonds present in: 1) double helices formed between AM and AMP chains, and 2) double helices forming the AMP clusters.

It was interesting to observe, that although $\Delta \mathrm{H}$ values were different for WP100 and WP120 starches (Table 4.5), both NP120 and NP100 starches exhibited nearly similar $\mathrm{AH}$ values (Table 4.5). This suggests that the presence of AM co-crystallized with AMP chains in NP starch may have resisted hydrogen bond disruption during HMT at $120^{\circ} \mathrm{C}$. Consequently, the extent of double helical disorganization may have been too small to be detectable by DSC. This seems plausible, since the K/S iodine absorption spectra showed that the difference in the extent of iodine absorption between NP and NP120 starches (Fig 4.9a) was smaller (due to a lower extent of double helical disruption on HMT) than between WP and WP120 starches (Fig 4.9b).

\subsection{Pasting properties}

The pasting properties of native and HMT normal and waxy potato starches measured using a Rapid Visco ${ }^{\mathrm{TM}}$ Analyser (RVA) are presented in Fig 4.10 and Table 4.6. All RVA parameters were higher in native normal potato starch. Studies have shown that peak viscosities are influenced by amylose content, extent of amylose leaching, granular swelling, friction between swollen granules, phosphate monoester content and/or the proportion of long amylopectin branch chains (Jayakody et al., 2007; Jane et al., 1999). As shown in Tables 4.1 and 4.2, there was no 
significant difference in phosphate monoester content and in the proportion of long amylopectin chains (DP 37-50), between native normal and waxy potato starches. Therefore, the difference in peak viscosity (normal>waxy) between the two native starches could be attributed to AML (normal potato) (Fig 4.6) negating the effect of granular swelling (waxy>normal) (Fig 4.7) on peak viscosity. McPherson and Jane (1999) also showed that normal potato starch displays a larger peak viscosity than waxy potato starch. This was attributed to AM-AMP interactions within native normal potato starch, which confers granule rigidity, thereby enabling granules to swell and to attain a higher peak viscosity than waxy potato starch.

Higher granule fragility (resulting from higher granular swelling) may have been responsible for the extent of viscosity breakdown being higher in normal potato starch (Fig 4.10). The higher set-back of native normal potato starch reflects more extensive amylose leaching (Fig 4.6). In both starches, HMT $\left(100,120,130^{\circ} \mathrm{C}\right)$ decreased peak viscosity (normal > waxy), breakdown viscosity (normal>waxy) and increased the pasting temperature (normal>waxy). Set-back decreased in normal potato starch, but increased in waxy potato starch on HMT (Fig 4.10). However, HMT at $80^{\circ} \mathrm{C}$ increased peak viscosity, breakdown viscosity, final viscosity and pasting temperature and decreased set-back in waxy potato starch. In normal potato starch, HMT at $80^{\circ} \mathrm{C}$ increased breakdown viscosity and decreased set-back. 


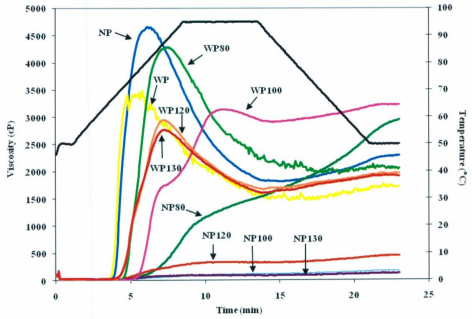


Table 4.6 RVA parameters* of native and heat-moisture treated normal and waxy potato starches

\begin{tabular}{|c|c|c|c|c|c|c|c|}
\hline Sample & $\begin{array}{l}\text { Peak } \\
(\mathrm{cP})\end{array}$ & $\begin{array}{l}\text { Trough } \\
\text { (cP) }\end{array}$ & $\begin{array}{c}\text { Breakdown } \\
\text { (cP) }\end{array}$ & $\begin{array}{c}\text { Final } \\
\text { viscosity } \\
\text { (cP) }\end{array}$ & $\begin{array}{c}\text { Setback } \\
\text { (cP) }\end{array}$ & $\begin{array}{l}\text { Peak Time } \\
\text { (min) }\end{array}$ & $\begin{array}{c}\text { Pasting } \mathrm{T}^{\circ} \\
\left({ }^{\circ} \mathrm{C}\right)\end{array}$ \\
\hline NP & $4605 \pm 75^{p}$ & $1800 \pm 14^{p}$ & $2805 \pm 61^{P}$ & $2277 \pm 14^{p}$ & $477 \pm 0.0^{P}$ & $6.23 \pm 0.1^{p}$ & $66.6 \pm 0.3^{p}$ \\
\hline NP80 & $1466 \pm 9^{4}$ & $1467 \pm 15^{4}$ & $-1 \pm 6^{\mathrm{q}}$ & $2937 \pm 18^{9}$ & $1470 \pm 3^{9}$ & $13.00 \pm 0.0^{9}$ & $84.3 \pm 0.0^{q}$ \\
\hline NP100 & $116 \pm 1^{t}$ & $108 \pm 1^{r}$ & $8 \pm 0^{r}$ & $165 \pm 0^{r}$ & $58 \pm 1^{\mathrm{r}}$ & $12.80 \pm 0.1^{4}$ & - \\
\hline NP120 & $332 \pm 1^{\mathrm{s}}$ & $317 \pm 2^{5}$ & $16 \pm 1^{s}$ & $449 \pm 1^{s}$ & $132 \pm 3^{5}$ & $11.90 \pm 0.1^{q}$ & - \\
\hline NP130 & $93 \pm 5^{1}$ & $78 \pm 1^{4}$ & $15 \pm 6^{5}$ & $121 \pm 3^{t}$ & $44 \pm 4^{t}$ & $9.13 \pm 0.0^{t}$ & - \\
\hline WP & $3506 \pm 29^{p}$ & $1462 \pm 3^{D}$ & $2044 \pm 26^{p}$ & $1693 \pm 31^{p}$ & $231 \pm 34^{p}$ & $5.30 \pm 0.7^{p}$ & $65.4 \pm 0.3^{p}$ \\
\hline WP80 & $4291 \pm 11^{4}$ & $1949 \pm 3^{9}$ & $2342 \pm 8^{9}$ & $2057 \pm 2^{4}$ & $108 \pm 5^{q}$ & $7.47 \pm 0.1^{4}$ & $70.7 \pm 0.0^{9}$ \\
\hline WP100 & $3119 \pm 30^{r}$ & $2884 \pm 32^{r}$ & $235 \pm 1^{r}$ & $3209 \pm 17^{r}$ & $326 \pm 15^{t}$ & $11.40 \pm 0.0^{4}$ & $76.6 \pm 0.0^{t}$ \\
\hline WP120 & $2978 \pm 51^{r}$ & $1691 \pm 35^{5}$ & $1287 \pm 16^{s}$ & $1982 \pm 28^{s}$ & $291 \pm 8^{5}$ & $7.27 \pm 0.1^{q}$ & $69.20 \pm 0.1^{s}$ \\
\hline WP130 & $2790 \pm 35^{5}$ & $1639 \pm 55^{5}$ & $1151 \pm 21^{t}$ & $1922 \pm 13^{1}$ & $283 \pm 42^{5}$ & $7.33 \pm 0.1^{4}$ & $69.53 \pm 0.0^{5}$ \\
\hline
\end{tabular}

*Values are means of two determinations. Means within each column with different superscripts for native and waxy potato starches and their heat moisture treated counterparts are significantly different $(\mathrm{P}<0.05)$ by Tukey's HSD test 
In normal potato starch, the reduction in peak viscosity (NP130 $>$ NP100 $>$ NP120 $>$ NP80) on HMT reflects decreased AML (Fig 4.6) and SF (Fig 4.7) as a result of enhanced interaction between $\mathrm{AM}-\mathrm{AM}, \mathrm{AM}-\mathrm{AMP}$ and $\mathrm{AMP}-\mathrm{AMP}$ chains and polymorphic transformation $(\mathrm{B} \rightarrow \mathrm{A}+\mathrm{B})$. Reduction of peak viscosity on HMT (WP130>WP120>WP100) in waxy potato starches could be attributed to lower granular swelling because of AMP-AMP interactions and polymorphic transformation $(B \rightarrow A+B)$. Reduction of peak viscosity is lower in waxy potato starch, since the extent of granular swelling is very high (Fig 4.7b). Starch interactions are also responsible for the increase in pasting temperature on HMT being higher in normal potato starch. Jayakody et al. (2007) have shown that both amylose gelation and the presence of rigid swollen granules embedded within the leached amylose network influence the extent of set-back. SF (Fig 4.7) and AML (Fig 4.6) data showed that HMT decreased the SF and AML of normal potato starch, but increased SF of waxy potato starch. This suggests that the increased setback on HMT of waxy potato (WP100, WP120, WP130) starch reflects an increase in the proportion of swollen intact granules (provides increased resistance to shearing during the cooling cycle in the RVA).

\subsection{Enzyme Hydrolysis}

Hydrolysis of native and HMT starches by PPA are presented in Figs 4.11. In their native state, NP was hydrolysed to a greater extent than WP starch. HMT at $80^{\circ} \mathrm{C}$, decreased hydrolysis in NP starch (Fig 4.11a) but did not significantly influence hydrolysis in WP starch (Fig 4.11b). However, HMT at 100,120 and $130^{\circ} \mathrm{C}$ increased hydrolysis of both NP and WP starches nearly to the same extent. The extent of this increase in NP and WP starches, was more pronounced between 120 and $130^{\circ} \mathrm{C}$. It is difficuit to compare these results with those reported in the literature for normal potato starch due to differences in moisture content, duration of HMT and 
HMT temperatures (Hoover, 2010). The granular architecture and, more specifically the surface organization has been shown to be one of the main factors determining kinetics and degree of hydrolysis (Bird et al., 2009). Thus the extent of decrease in molecular order that occurs at or near the granule surface on HMT of NP starch (NP130-NP120>NP100>NP80) (Table 4.4) should have theoretically increased hydrolysis in the same order. However, the observed order of hydrolysis (NP130 $>$ NP120 $>$ NP1 $100 \mathrm{NP}>\mathrm{NP} 80$ ), suggests that interactions between starch chains on HMT (Fig 4.6), which are more prevalent at 80 and $100^{\circ} \mathrm{C}\left(80>100^{\circ} \mathrm{C}\right.$ ) than at 120 and $130^{\circ} \mathrm{C}$ may have also been a contributory factor influencing hydrolysis. At this stage, it might be instructive to consider the conformational changes in glucosyl residues during PPA hydrolysis. Oates (1997) has shown that PPA catalyzed hydroiysis of $\alpha(1 \rightarrow 4)$ glycosidic bonds involves the enzyme -induced ring distortion of one of the D-glucosyl residues from the ${ }^{4} \mathrm{C}_{1}$ chair conformation to a 'haif chair' conformation. Ring distortion decreases the enthalpy of activation and increases the susceptibility of the glucosyl residues to hydrolysis. The ability of PPA to unwind double helices is driven by the influence of ring distortion on neighbouring units. Chains with restricted mobility, either complexed or crystallized will be hydrolysed less readily because their constituent glucose units are more firmiy locked into a specific configuration.

Jane et al. (1997) have shown that in A-type starches, the branch points are scattered in both amorphous and crystalline regions. Consequently, there are many short chains derived from branch linkages located inside the crystalline region, which produces an inferior crystalline structure. This inferior crystalline structure containing $\alpha(1 \rightarrow 6)$ branch points and the short double helices are more susceptible to PPA hydrolysis leading to a 'weak points' in starches containing the A-type unit cells. These weak points are readily attacked by PPA. 

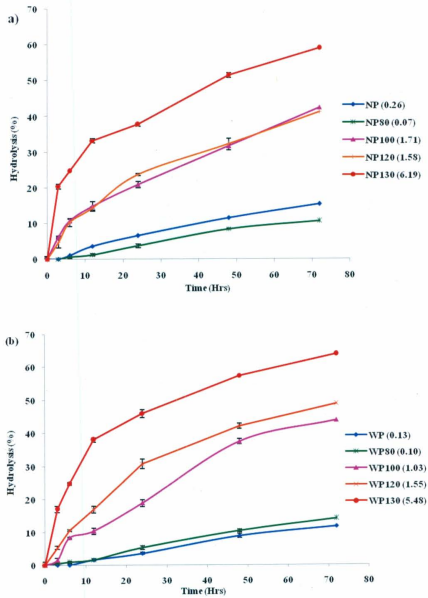
However, in B-type starches more branch points are clustered in the amorphous region, and furthermore, there are fewer short branch chains. Consequently, the crystalline structure is superior to that of the A-type starches, and hence more resistance to PPA. Ratnayake et al. (2001) have shown by studies on pulse starches that resistance to PPA increases with increase in B-type unit cell content. X-ray data (Fig 4.5) showed the formation of A-type unit cells on HMT of NP starch at $80^{\circ} \mathrm{C}$. ATR-FTIR data (Table 4.4) indicated structural disorganization of starch chains near the granule surface of NP80 starch. Thus, both of the above factors should have theoretically increased the susceptibility of NP80 starch towards PPA. The observed hydrolysis (NP>NP80), suggests that the extensive interaction between starch chains (AM-AM, AM-AMP) during HMT at $80^{\circ} \mathrm{C}$ (Fig 4.6) may have hindered the accessibility of PPA towards the glycosidic oxygen. This suggests that the structural disorganization near the granule surface as observed by ATR-FTIR data (Table 4.4) mainly reflects disorganization of AMP helices. The large increase in hydrolysis on HMT of NP starch at $100^{\circ} \mathrm{C}$ (Fig $4.11 \mathrm{a}$ ) reflects an increase in Atype crystallites (Fig 4.6) and greater degree of structural disorganization near the granule surface (Table 4.4). This seems plausible, since the extent of interaction involving AM chains at $100^{\circ} \mathrm{C}$ was much weaker than at $80^{\circ} \mathrm{C}$ (Fig 4.6). The marginal difference in hydrolysis between NP100 and NP120 starches was rather surprising, since compared to NP100 starch, NP120 starch exhibited higher structural disorganization near the granule surface (Table 4.4), weaker interaction between AM-AM and AMP-AMP chains (Fig 4.7) and disruption of hydrogen bonds between co-crystallized AM and AMP chains (Table 4.5). Consequently, NP120 starch should have been hydrolysed to a greater extent than NP100 starch. This suggests that the increase in the amount of A-type unit cells (NP120>NP100) formed on HMT (Fig 4.5, Table 4.3) may have been the causative factor influencing the difference in hydrolysis between NP120 and NP100 
starches. Several studies (Lopez-Rubio et al., 2008b; O'Brien and Wang, 2008; Oates 1997) have shown that the double helical conformation hinders enzyme hydrolysis, since in this form only a maximum linear portion of three glucose units is available; this is insufficient for complete subsite binding. This suggests that the large difference in hydrolysis between NP120 and NP130 starches (Fig 4.11a,b) reflects helix to coil transformation and the greater content of A-type unit cells in the latter (Fig 4.5, Table 4.3).

X-ray (Fig 4.5, Table 4.3) and ATR-FTIR (Table 4.4) data showed an increase in A-type crystallites and structural disorganization (near the granule surface), respectively, on HMT of WP starch at $80^{\circ} \mathrm{C}$. On this basis, WP8 0 starch should have been hydrolysed to a greater extent than WP starch. The nearly similar hydrolysis values for WP and WP80 starches (Fig 4.11b) is indicative that AMP-AMP interactions during HMT at $80^{\circ} \mathrm{C}$, may have negated the expected increase in hydrolysis by hindering the accessibility of glycosidic oxygen to PPA. In comparison with NP100 and NP120 starches, the difference in hydrolysis between WP100 and WP120 starch (WP120 $>$ WP100) was very significant (Fig 4.11b). This could be attributed to greater disruption of $\mathrm{H}$-bonds between AMP chains at $120^{\circ} \mathrm{C}$, which renders the AMP chains more susceptible to $\alpha$-amylolysis ( $\sec 4.8$ ). The large difference in hydrolysis between WP120 and WP130 starches reflects the presence of more A-type crystallites (Fig 4.5, Table 4.3) and to the presence of interrupted helices in the latter ( $\sec 4.8)$. The results indicate that reactivity of PPA towards HMT starches is influenced by the interplay of :1) interactions between starch chains, 2) structural disorganization near the granular surface, 3) amount of A-type crystallites, 4) double helical organization within the AMP crystallites. 
Bright field (BFM) and cross-polarized light microscopy of (CPLM) of PPA hydrolysed (24h) residues of NP and WP starches before and after HMT at $100^{\circ}$ and $130^{\circ} \mathrm{C}$ are presented in Fig 4.12. CPLM showed decreased birefringence intensity at the granule centre of both hydrolysed residues of NP (Fig 4.12A) and WP (Fig 4.12B) starches. BFM (Fig 4.12A) and CLSM (Fig 4.13) showed peripheral exocorrosion in hydrolysed NP starch. Whereas, SEM (Fig 4.14) showed the presence of pitting and roughened surface that varied from granule to granule. Hydrolysed WP starch showed extensive splitting (Fig 4.12B, Fig 4.13) but very little peripheral exocorrosion. SEM of hydrolysed WP starcin (Fig 4.14) revealed the presence of numerous pores that were larger in diameter than the pores present in unhydrolysed granules (Fig 4.1) (largely confined to the exterior regions of the granule). Granule splitting and roughened surfaces were also seen in many granules (Fig 4.14). 
Fig 4.12 Microscopy of hydrolysed (pancreatic $\alpha$-amylase/24h) residues of native and HMT $\left(100^{\circ}\right.$ and $\left.130^{\circ} \mathrm{C}\right)$ normal potato (NP) [A] and waxy potato (WP) [B] starches. For NP and WP starches the same field is shown for bright field (upper frame) and between cross polarized light (lower frame). 


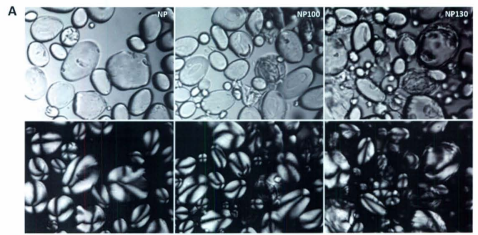

B

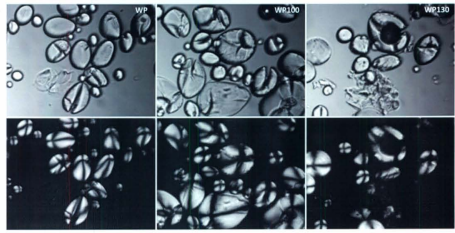


Fig 4.13 Confocal laser scanning microscopy (APTS stained) of porcine pancreatic $\alpha$-amylase (PPA) hydrolysed (24h) granules of native normal (NP), NP heat-moisture treated at $100^{\circ} \mathrm{C}$ (NP100), NP heat-moisture treated at $130^{\circ} \mathrm{C}$ (NP130), native waxy potato (WP), WP heat-moisture treated at $100^{\circ} \mathrm{C}$ (WP100) and WP heat-moisture treated at $130^{\circ} \mathrm{C}$ (WP130). 


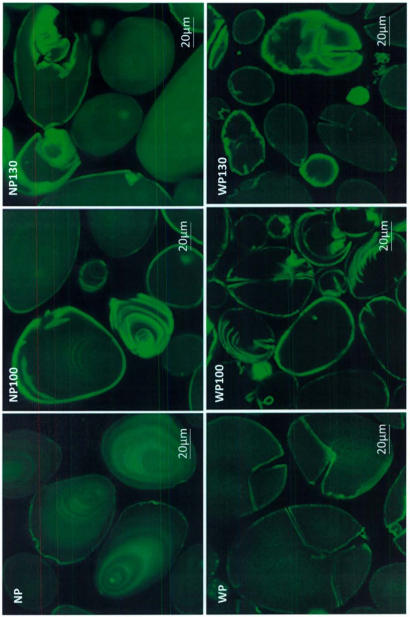


Fig 4.14 Scanning electron microscopy of porcine pancreatic $\alpha$-amylase (PPA) hydrolysed (24h) granules of native normal (NP), NP heat-moisture treated at $100^{\circ} \mathrm{C}$ (NP100), NP heat-moisture treated at $130^{\circ} \mathrm{C}$ (NP130), native waxy potato (WP), WP heat-moisture treated at $100^{\circ} \mathrm{C}$ (WP100) and WP heat-moisture treated at $130^{\circ} \mathrm{C}$ (WP130). 

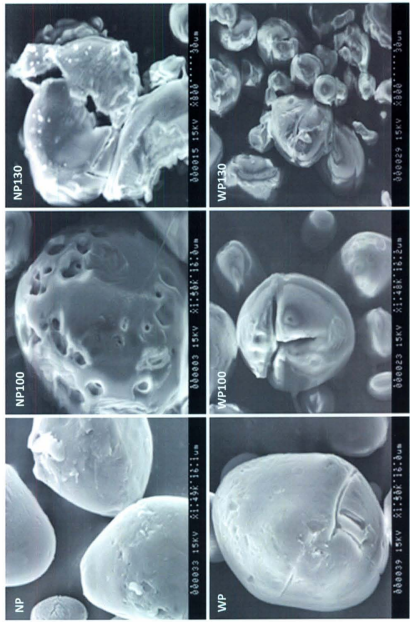
Blazec and Copeland (2010) have shown by studies on waxy and high amylose wheat starches that growth ring structures are more readily digested in waxy starch granules than in amylose rich maize starches. They attributed this to the absence of amylose in the amorphous layers of waxy starch granules that would allow them to absorb water more readily, thereby facilitating the entry of $\alpha$-amylase into the granule interior. This suggest that the difference in granule morphology between hydrolysed NP and WP starches could be attributed both to the presence of pores and higher granular swelling (Fig $4.7 \mathrm{~b}, \mathrm{c}$ ) in WP starch. In both NP and WP starches, the loss in birefringence intensity on hydrolysis was more pronounced after HMT (Fig 4.12A, B). BFM of NP100 starch showed increased exocorrosion, and some degree of internal degradation near the hilum. A few granules of NP100 starch were deformed (Fig 4.11A). However, granules of WP100 starch (Fig 4.12B) exhibited extensive splitting. The extent of splitting varied among the granules (Fig 4.12B). SEM of NP100 starch showed numerous large pores on the granule surface, which appeared spongy. Whereas, WP100 starch showed many split granules. CLSM of NP100 starch revealed more extensive peripheral exocorrosion than in NP starch and some degree of internal degradation (Fig 4.13). Whereas, WP100 showed many split granules and peripheral exocorrosion. In both NP130 and WP130 starches, the loss in birefringence intensity was more pronounced than at $100^{\circ} \mathrm{C}$ (Fig 4.12A, B). BFM showed that granules of both NP130 (Fig 4.12A) and WP130 (Fig 4.12B) starches were more extensively degraded than at $100^{\circ} \mathrm{C}$. Granules of NP130 starch showed the presence of holes on granule surfaces, internal degradation and granule deformation (Fig 4.12A). However, in WP130 starch, granules were split and some were disintegrated (Fig 4.12B). SEM revealed granule splitting in some granules of NP130 and WP130 starches (Fig 4.14). The extent of this splitting being more pronounced in the latter. CLSM of NP130 and WP130 starches (Fig 4.13), revealed extensive degradation near the hilum, 
whereas, some granules were not degraded. The above data showed that granules of both NP and WP starches exhibited heterogeneity in degradation (NP>WP) in their native and HMT states. This could reflect differences within each granule with respect to: 1) molecular order near the granule surface, 2) variation in granule size, 3) extent to which B-type crystallites were converted to A-type crystallites during HMT, 4) extent of disorganization of the region surrounding the hilum during HMT, 5) extent to which double helical chains unravel during HMT and 6) arrangement of amylose and amylopectin within the granule interior. The greater level of heterogeneity in NP starch could be attributed to its higher amylose content. 


\section{CHAPTER 5}

\section{SUMMARY AND CONCLUSIONS}

The data obtained from X-Ray, ATR-FTIR, K/S spectra, SF, AML, DSC, RVA showed that structural and property changes on HMT of NP and WP starches were mainly influenced by differences in starch chain mobility at the different temperatures of HMT. Starch chain mobility, in turn was influenced by the interplay between the extent to which B-type crystallites were transformed into $\mathrm{A}+\mathrm{B}$-type crystallites and the kinetic energy imparted to starch chains.

HMT at all temperatures $\left(80,100,120\right.$ and $\left.130^{\circ} \mathrm{C}\right)$ changed the $\mathrm{X}$-ray pattern $(\mathrm{B} \rightarrow \mathrm{A}+\mathrm{B})$ of both NP and WP. The extent of this transformation increased with increase in HMT temperature. The decrease in AML and K/S intensity on HMT at low temperatures $\left(80-100^{\circ} \mathrm{C}\right)$ can be attributed to additional interaction between starch chains. The increase in AML on HMT at higher temperatures $\left(120\right.$ and $\left.130^{\circ} \mathrm{C}\right)$ is indicative that at $120^{\circ} \mathrm{C}$ and $130^{\circ} \mathrm{C}$, hydrogen bonds between co-crystallized AM and AMP chains may have disrupted. The decrease in $\triangle \mathrm{H}$ at $120^{\circ}$ and $130^{\circ} \mathrm{C}$ reflect mainly disruption of hydrogen bonds present in: 1) double helices formed between AM and AMP chains, and 2) double helices forming the AMP clusters. Lower K/S maxima of NP130 starch and the shift to a lower $\lambda_{\max }(570 \mathrm{~nm})$, suggests that intra molecular hydrogen bonds stabilizing helical region may have disrupted on HMT at $130^{\circ} \mathrm{C}$. AMP chain length distribution of both native and HMT starches remained unchanged at all HMT temperatures. This suggests that covalent linkages of AMP were not broken under the HMT conditions $\left(27 \%\right.$ moisture, $\left.16 \mathrm{~h}, 80-130^{\circ} \mathrm{C}\right)$ used in this study. 
Therefore, the main type of structural changes that had great impact on physicochemical properties at different temperatures of HMT were polymorphic transition $(B \rightarrow A+B), A M-A M$, AM-AMP and AMP-AMP interactions $\left(80\right.$ and $\left.100^{\circ} \mathrm{C}\right)$, disruption of hydrogen bonds between co-crystallized AM and AMP chains and between AMP-AMP chains $\left(120\right.$ and $\left.130^{\circ} \mathrm{C}\right)$, disorganization of AMP chains near the vicinity of the hilum $\left(100,120\right.$ and $\left.130^{\circ} \mathrm{C}\right)$, and formation of interrupted helices $\left(130^{\circ} \mathrm{C}\right)$. The extent of changes to starch structure and properties on HMT was different in normal and waxy starches due to differences in their amylose content and amylopectin chain mobility.

The susceptibility of NP and WP starches towards PPA hydrolysis decreased with HMT at $80^{\circ} \mathrm{C}$, but increased with HMT at $100-130^{\circ} \mathrm{C}$. This suggested that PPA hydrolysis of HMT starches was influenced by the interplay of: 1) the amount of A-type crystallites, 2) starch chain interactions and 3) changes to the double helical conformation. There are differences between the mode of attack by pancreatic $\alpha$-amylase on NP and WP starch granules. These differences in granule morphology in PPA hydrolyzed NP and WP starches were largely influenced by the presence of pores and higher granular swelling in the latter. NP and WP starches exhibited heterogeneity in degradation (NP>WP) in both their native and HMT states. This suggests that within each granule, variations exist with respect to molecular order, double helical packing arrangement of AM and AMP and the proportion of A- to B-type unit cells. 


\section{DIRECTIONS FOR FUTURE RESEARCH}

- Heat-moisture treatment of starch is of interest due to its possible applications in the food industry, either for use alone or in combination with other types of starch modifications. Studies showed that HMT changes starch structure and its physicochemical properties. The type and extent of these changes are influenced by starch source, starch composition and treatment conditions. However, the exact molecular mechanisms of HMT still remains poorly understood. This study provided only a partial glimpse into the molecular mechanism of HMT. In order to further understand the molecular mechanism of HMT, experiments must be conducted at different levels of time/moisture/temperature combinations on starches varying widely in amylose content (maize, barley and rice).

- Presently, there is an interest in developing starches with modified digestion (slowly digestible starch [SDS], and resistant starch [RS]) characteristics. These modified products have the potential to benefit human health through moderation of the glycemic response and as a source of prebiotic carbohydrate. Results showed that starch digestibility is influenced by HMT temperature. Thus, HMT may be a tool for modifying starch digestibility.

- Acid hydrolysis has been used as a tool to modify starch properties. This is due to the preferential attack of $\mathrm{H}_{3} \mathrm{O}^{+}$on the amorphous domain. Thus, it would be worthwhile to investigate how acid hydrolysis followed by HMT and HMT followed by acid hydrolysis would influence starch properties in normal, waxy and high amylose starches. 


\section{REFERENCES}

AACC (2000). Approved methods of the AACC (10 ed.), St. Paul, MN, USA: American Association of Cereal Chemists.

Abraham, T. E. (1993). Stabilization of paste viscosity of cassava starch by heat-moisture treatment. Starch, 43, 131-135.

Absar, N., Zaidul, I. S. M., Takigawa, S., Hashimoto, N., Matsuura-Endo, C., Yamauchi, H. and Noda, T. (2009). Enzymatic hydrolysis of potato starches containing different amounts of phosphorus. Food Chemistry, 112, 57-62.

Acker, L. (1977). The lipids of starch—Research between carbohydrates and lipids. Fette Seifen Anstrichmittel, 79, 1-9.

Adebowale, K. O. and Lawal, O, S. (2003). Microstructure, physicochemical properties and retrogradation behaviour of mucuna bean (Mucuna puriens) starch on heat moisture treatment. Food Hydrocolloid, 17, 265-272.

Adebowale, K. O., Afolabi, T. A. and Olu-Owolabi, B. I. (2005a). Hydrothermal treatment of finger millet (Eleusine coracana) starch. Food Hydrocolloids, 19, 974-983

Adebowale, K. O., Afolabi, T. A., Olu-Owolabi, B. I., Olawum, E. K., and Lawal, O. S. (2005b). Functional properties of native, physically and chemically modified breadfruit (Artocarpus artilis) starch. Industrial Crops and Products, 21, 343-351.

Anderson, A. K., Guraya, H. S. (2006). Effects of microwave heat-moisture treatment on properties of waxy and non-waxy rice starches. Food Chemistry, 97, 318-323.

Anderson, A. K., Guraya, H. S., James, C. and Salvaggio, L. (2002). Digestibility and pasting properties of rice starch heat-moisture treated at the melting temperature $(T \mathrm{~m})$. Starch, $54,401-409$.

Ao, Z., Simsek, S., Zhang, G., Venkatachalam, M., Reuhs, B. L. and Hamaker, B. R. (2007). Starch with a slow digestion property produced by altering its chain length, branch density, and crystalline structure. Journal of Agricultural and Food Chemistry, 55, 4540-4547. 
Atkin, N. J., Cheng, S. L., Abeysekera, R. M. and Robards, A. W. (1999). Localisation of amylose and amylopectin in starch granules using enzyme-gold labeling. Starch, 51, 163-172.

Atwell, W. A., Hood, L. F., Lineback, D. R., Varriano-Marston, E. and Zobel, H. E. (1988). The terminology and methodology associated with basic starch phenomena. Cereal Foods World, 33, 306-311.

Badenhuizen, N.P. (1969). The biogenesis of starch granules in higher plants. Appleton Crofts, NY.

Bailey, 1. M. and Whelan, W. J. (1961). Physical properties of starch. Journal of Biological Chemistry, 236, 969-973.

Baker, A. A., Miles, M. J. and Helbert, W. (2001). Internal structure of the starch granule revealed by AFM. Carbohydrate Research, 330, 249-256.

Baker, L. A. and Rayas-Duarte, P. (1998). Freeze-thaw stability of amaranth starch and the effects of salt and sugars. Cereal Chemistry, 75, 301-303.

Baldwin, P. M., Davies, M. C., and Melia, C. D. (1997). Starch granule surface imaging using low-voltage scanning electron microscopy and atomic force microscopy. Biological Macromolecules, 21, 103-107.

Banks, W. and Greenwood, C. T. (1967). The fractionation of laboratory- isolated cereal starches using dimethyl sulphoxide. Starch, 19, 394-398.

Banks, W. and Greenwood, C. T. (1971). Amylose: a non helical biopolymer in aqueous solution. Polymer, 12, 141-145

Banks, W. and Greenwood, C. T. (1975). Starch and its Components, Chapter 4, Edinburgh University Press, Edinburgh

Banks, W., Greenwood, C. T. and Khan, K. M. (1971). The interaction of linear amylose oligomers with iodine. Carbohydrate Research, 17, 25-33. 
Baum, B. R. and Bailey, L. G. (1987). A survey of endosperm starch granules in the genus Hodeum: a study using image analytic and numerical taxonomic techniques. Canadian Journal of Botany, 65, 1563-1569.

Bay-Smidt, A. M., Wischmann, B., Olsen, C. E. and Nielsen, T. H. (1994). Starch bound phosphate in potato as studied by a simple method for determination of organic phosphate and 31P-NMR. Starch, 46, 167-172.

Benmoussa, M., Hamaker., B. C., Huang, C. P., Sherman, D. M., Weil, C. F. and BeMiller, J. N. (2010). Elucidation of maize endosperm starch granule channel proteins and evidence for plastoskeletal structures in maize endosperm amyloplasts. Journal of Cereal Science, $52,22-29$

Bertoft, E. (2004). Lintnerization of two amylose-free starches of A- and B- crystalline types, respectively. Starch, 56, 167-180.

Bertoft, E. (1991). Investigation of the fine structure of alpha-dextrins derived from amylopectin and their relation to the structure of waxy-maize starch. Carbohydrate Research, 212, 229-244.

Bertoft, E. and Manelius, R. (1992). A method for the study of the enzymic hydrolysis of starch granules. Carbohydrate Research, 227, 269-283.

Bertoft, E. and Qin, Z. (1993). Studies on the Structure of Pea Starches Part 4: Intermediate material of wrinkled pea starch. Starch, 45, 420-425.

Bijttebier, A., Goesaert, H. and Delcour, J. A. (2008). Amylase action pattern on starch polymers. Biologia, 63, 989-999.

Biliaderis (1998). Structures and phase transitions of starch polymers. In R. H. Walter (Ed), Polysaccharide association structures in food, (pp. 57-168). Marcell Dekker, Inc. NY

Biliaderis, C. G. (1992). Structures and phase transitions of starch in food systems. Food Technology, 46, 98-109. 
Biliaderis, C. G. (2009). Structural transitions and related physical properties of starch. In J. BeMiller, and R. Whistler (Eds), Starch:Chemistry and Technology, (pp 293-359). Academic Press, NY.

Biliaderis, C. G. and Seneviratne, H. D. (1990). On the supermolecular structure and metastability of glycerol monostearate-amylose complex. Carbohydrate Polymers, 13, 185-206.

Biliaderis, C. G. Grant, D. R. and Vose, J. R. (1981). Structure characterization of legume starches. II. Studies on acid treated starches. Cereal Chemistry, 58, 502-507.

Biliaderis, C. G. and Galloway, G. (1989). Crystallization behaviour of amylose-V complexes: structure-property relationships. Carbohydrate Research, 189, 31-48.

Biliaderis, C. G. and Seneviratne, H. D. (1990). On the supermolecular structure and metastability of glycerol monostearate-amylose complex. Carbohydrate Polymers, 13, 185-206.

Biliaderis, C. G., Page, C. M., Maurice, T. J. and Juliano, B. O. (1986a). Thermal characterization of rice starches: a polymeric approach to phase transition of granular starch. Journal of Agricultural and Food Chemistry, 34, 6-14.

Biliaderis, C. G., Tonogai, J. R., Perez, C. M. and Juliano, B. O. (1993), Thermophysical properties of milled rice starch as influenced by variety and parboiling method. Cereal Chemistry, 70, 512-516.

Biliaderis, C. G. (1998). Structures and phase transitions of starch polymers. In R. H. Walter, (Ed), Polysaccharide Association Structures in Foods, (pp. 57-168). Marcel Dekker, NY.

Biliaderis, C. G., Page, C. M. and Maurice, T. J. (1986b). Non-equilibrium melting of amyloseV complexes. Carbohydrate Polymers, 6, 269-288.

Bird, A. R., Lopez-Rubio, A., Shrestha, A. K. and Gidley, M. J. (2009). Resistant starch in vitro and in vivo: Factors determining yield, structure, and physiological relevance. In S. 
Kasapis, I. T. Norton, and J. B. Ubbink (Eds.), Modern biopolymer sciences (pp. 449-512). Academic Press, London.

Blanshard, J. M. V. (1987). Starch granule structure and function: a physicochemical approach. In T. Galliard (Ed.), Starch: Properties and potential (pp. 16). Chichester: John Wiley and Sons.

Blaszczak, W. Fomal, J. and Amarowicz, R. (2003). Lipids of wheat, com and potato starch. Journal of Food Lipids, 10, 301-312.

Blazeck, J. and Copeland, L. (2010). Amylolysis of wheat starches. II. Degradation pattern of native starch granules with varying functional properties. Journal of Cereal science, doi:10.1016/j.jcs.2010.06.011.

Blennow, A., Bay-Smidt, A. M., Olsen, C. E. and Møller, B. L. (2000). The distribution of covalently bound phosphate in the starch granule in relation to starch crystallinity. International Journal of Biological Macromolecules, 27, 211-218.

Blennow, A., Bay-Smidt, A. M., Wischmann, B., Olsen, C. E. and Lindberg-Møller, B. (1998). The degree of starch phosphorylation is related to the chain length distribution of the neutral and the phosphorylated chains of amylopectin. Carbohydrate Research, 307, 4554.

Blennow, A., Hansen, M., Schulz, A., Jørgensen, K., Donald, A. M. and Sanderson, J. (2003). The molecular deposition of transgenically modified starch in the starch granule as imaged by functional microscopy. Journal of Structural Biology, 143, 229-241

Bogracheva, T. Y. Wang Y. L. and Hedley, C. L. (2001). The effect of water content on the ordered/disordered structures in starches. Biopolymers, 58, 247-259.

Bogracheva, T. Y., Morris, V, J., Ring, S. G. and Hedley, C. L. (1998). The granular structure of C-type pea starch and its role in gelatinization. Biopolymers, 45, 323-332.

Brand, J. C., Colagiuri, S., Crossman, S., Allen, A. and Truswell, A. S. (1991). Low glycemic index carbohydrate foods improve glucose control in non-insulin dependent diabetes mellitus (NIDDM). Diabetes Care 14, 95-101. 
Brumovsky, L. A., Brumovsky, J. O., Fretes, M. R. and Peralta, J. M. (2009). Quantification of resistant starch in several starch sources treated thermally. International Journal of Food Properties, 12, 451-460.

Brumovsky, J. O. and Thompson,D. B. (2001). Production of boiling stable granular resistant starch by partial acid hydrolysis and hydrothermal treatments of high amylose maize starch. Cereal Chemistry, 79,680-689.

Bruner, R. L. (1964). Determination of reducing value. In R.L. Whistler (Ed), Methods in Carbohydrate Chemistry: Starch (Vol. IV, pp. 67-71). Academic Press, NY.

Buléon, A. Colonna, P. Planchot V. and Ball, S. (1998). Starch granules: Structure and biosynthesis. International Journal of Biological Macromolecules 23, 85-112.

Buléon, A., Bizot, H., Delage, M. M. and Multon, J. L. (1982). Evolution of Crystallinity and Specific Gravity of Potato Starch versus Water Ad- and Desorption. Starch, 34, 361-366.

Bulpin, P. V., Welsh, E. J. and Morris, E. R. (1982). Physical characterization of amylose-fatty acid complexes in granules and in solution. Starch, 34, 335 .

Burrell, M. M. (2003). Starch: the need for improved quality or quantity - an overview.Journal of Experimental Botany, 54,451-456.

Cairns, P., Bogracheva, T. Y., Ring, S. G., Hedley, C. L. and Morris, V. J. (1997). Determination of the polymorphic composition of smooth pea starch. Carbohydrate Polymers, 32, 275-282.

Calabrese, V. T. and Khan, A., (1999). Amylose-iodine complex formation without KI: evidence for absence of iodide ions within the complex. Journal of Polymer Science: Part A, 37, 2711-2717.

Cameron, R. E. and Donald, A. M., (1992). A small-angle X-ray scattering study of the annealing and gelatinisation of starch. Polymer, 33, 2628-2635. 
Cao, H. and Shannon, J. C. (1997). BT1, a possible adenylate translocator, is developmentally expressed in maize endosperm but not detected in starchy tissues from several other species. Physiologia Plantarum, 100, 400-406.

Capron, L, Robert, P., Colonna, P., Brogly, M. and Planchot, V. (2007). Starch in rubbery and glassy states by FTIR spectroscopy. Carbohydrate Polymers, 68, 249-259.

Cardoso, M. B., Putaux, J. L., Nishiyama, Y., Helbert, W., Hytch, M., Silveira, N. P. and Chanzy, H. (2007), Single crystals of V-amylose complexed with $a$-naphthol Biomacromolecules, 8, 1319-1326.

Carlson, T. L. G., Larsson, K., Dinh-Nguyen, N. and Krog, N. (1979). A study of the amylosemonoglyceride complex by Raman spectroscopy. Starch, 31, 222-224.

Cheetham, N. W. H. and Tao, L. P. (1998a). Solid state NMR studies on the structural and conformational properties of natural maize starches. Carbohydrate Polymers, 36, 285292.

Cheetham, N. W. H. and Tao, L. P. (1998b) Amylose conformational transitions in binary DMSO/water mixtures. Carbohydrate Polymers, 35, 287-295.

Chung, H. J., Hoover, R. and Liu, Q. (2009b). The impact of single and dual hydrothermal modifications on the molecular structure and physicochemical properties of normal corn starch. International Journal of Biological Macromolecules, 44, 203-210.

Chung, H. J., Liu, Q. and Hoover, R. (2009a). Impact of annealing and heat-moisture treatment on rapidly digestible, slowly digestible and resistant starch levels in native and gelatinized corn, pea and lentil starches. Carbohydrate Polymers, 75, 436-447.

Chung, H. J., Liu, Q. and Hoover, R. (2010). Effect of single and dual hydrothermal treatments on the crystalline structure, thermal properties and nutritional fractions of pea, lentil and navy bean starches. Carbohydrate Polymers, 43, 501-508.

Cleven, R., van den Berg, C. and van der Plas, L. (1978). Crystal Structure of Hydrated Potato Starch. Starch, 30, 223-228. 
Collado, L. S. and Corke, H. (1999). Heat-moisture treatment effects on sweet potato starches differing in amylose content. Food Chemistry, 65, 339-346

Colonna, P. and Buleon, A. (2009). Thermal transitions of starches. In A. C. Bertolini, (Ed), Starches: Characterization, properties and applications, (pp 72-95). CRC Press, Boca Raton, FL.

Colonna, P., Buléon, A. and Lemarié, F. (1988), Action of Bacillus subtilis $\alpha$-amylase on native wheat starch. Biotechnology and Bioengineering, 31, 895-904.

Colonna, P., Leloup, V. and Buleon, A. (1992). Limiting factors of starch hydrolysis, European Journal of Clinical Nutrition, 46, S17-\$32.

Cooke, D. and Gidley, M. (1992). Loss of crystallinity and molecular order during starch gelatinization: origin of the enthalpic transition. Carbohydrate Research, 227, 103-112.

Copeland, L., Blazek, J., Salman, H. and Tang, M. C. (2009). Form and functionality of starch, Food Hydrocolloids, 23, 1527-1534

Cowie, J. M. G. (1961). Studies on amylose and its derivatives. I. Molecular size and configuration of amylose molecules in various solvents. Die Makromoleculare Chemie, $42,230-247$.

Craig, S. A. S., Maningat, C. C., Seib, P. A. and Hoseney, R. C. (1989). Starch paste clarity. Cereal Chemistry 66, 173-182.

Davis, H. and Khan, A. (1994). Determining the chromophore in the amylopectin-iodine complex by theoretical and experimental studies. Journal of Polymer Science Part A: Polymer Chemistry, 32, 2257-2265.

Davis, H., Skrzypek, W. and Khan, A. (1994). Iodine binding by amylopectin and stability of the amylopectin-iodine complex. Journal of Polymer Science Part A: Polymer Chemistry, $32,2267-2274$.

Davis, J. P., Supatcharee, N., Khanddelwal, R. L. and Chibbar, R. N. (2003). Synthesis of novel starches in Planta: Opportunities and challenges. Starch, 55, 107-120. 
Debet, M. R. and Gidley, M. J. (2006). Three classes of starch granule swelling: Influence of surface proteins and lipids. Carbohydrate Polymers, 64, 452-465.

Dona, A. C., pages, G., Gilbert, R. G. and Kuchel, P. W. (2010). Digestion of starch: In vivo and in vitro kinetic models used to characterise oligosaccharide or glucose release. Carbohydrate Polymers, 80, 599-617.

Donald, A. M. (2004). Understanding starch structure and functionality. In: A-C. Eliasson, (Ed), Starch in food: Structure, function and applications (pp. 156-184). Woodhead Publishing Limited/CRC Press LLC, Cambridge/Boca Raton.

Donald, A. M. Kato, K. L., Perry, A. P and Waigh, T. A. (2001). Scattering studies of internal structure of starch granules. Starch, 53,504-512.

Donovan, J. W. (1979). Phase transitions of the starch-water system. Biopolymers 18, 263-275.

Donovan, J. W. and Mapes, C. J. (1980). Multiple phase transitions of starches and Naegeli amylodextrins. Starch, 32,190-193.

Doublier, J. L. (1987). A rheological comparison of wheat, maize, faba bean and smooth pea starches. Journal of Cereal Science, 5, 247-262.

Eerlingen, R. C., Jacobs, H., Block, K. and Delcour, J. A. (1997). Effects of hydrothermal treatments on the rheological properties of potato starch. Carbohydrate Research, 297, $347-356$

Eerlingen, R.C., Jacobs, H., Van Win, H. and Delcour, J. A. (1996). Effect of hydrothermal treatment on the gelatinization properties of potato starch as measured by differential scanning calorimetry. Journal of Thermal Analysis, 47, 1229-1246.

ELIANETM (2006), http://www.agfdt.de/loads/st06/semeiabb.pdf (2010-09-28).

Eliasson, A. C. and Gudmundsson, M. (1996). Starch: physicochemical and functional aspects. In A. C. Eliasson, (Ed), Carbohydrates in food (pp. 431-503). Marcel Dekker.

Eliasson, A. C. and Krog, N. (1985). Physical properties of amylose-monoglyceride complexes. Journal of Cereal Science, 3, 239-248. 
Englyst, H. N., Kingman, S. M. and Cummings, J. H. (1992). Classification and measurement of nutritionally important starch fractions. European Journal of Clinical Nutrition, 46: $533-550$,

Eppendorfer, W. H., Eggum, B. O. and Bille, S. W. (1979). Nutritive value of potato crude protein as influenced by manuring and amino acid composition. Journal of Science Food and Agriculture 30, 361-368.

Evans, A. and Thompson, D. B. (2004). Resistance to amylase digestion in four native highamylose maize starches. Cereal Chemistry 81, 31-37.

Evans, A., McNish, N. and Thompson, D. B. (2003). Polarization colors of lightly iodinestained maize starch for amylose-extender and related genotypes in the W64A inbred line. Starch, 55, 250-257.

Evans, I. D. and Haisman, D. R. (1982). The effect of solutes on the gelatinization temperature range of potato starch. Starch, 34, 224-231.

Evans, I. D., and Haisman, D. R. (1979). Rheology of gelatinized starch suspensions. Journal of Textural Studies, 10,347-370.

Evers, A. D. and Juliano, B. O. (1976). Varietal Differences in Surface Ultrastructure of Endosperm Cells and Starch Granules of Rice. Starch, 28, 160-166.

Fannon J. E., Hauber R. J., and BeMiller J. N., 1992. Surface pores of starch granules. Cereal Chemistry, 69, 284-288.

Fannon, J. E., Gray, J. A., Gunawan, N., Huber, K. C. and BeMiller, J. N. (2004). Heterogeneity of starch granules and the effect of granule channelization on starch modification. Cellulose, 11, 247-254.

Fannon, J. E., Shull, J. M. and BeMiller, J. N. (1993). Interior channels of starch granules. Cereal Chemistry, 70, 611-613.

Franco, C. M. L., Ciacco, C. F. and Tavares, D. Q. (1995). Effect of the heat-moisture treatment on the enzymatic susceptibility of corn starch granules, Starch, 47, 223-228. 
French, D. (1972). Fine structure of starch and its relationship to the organization of starch granules. Starch 19,8-25.

French, D. (1984). Organization of starch granules. In R. L. Whistler, J. N. Bemiller, and E. F. Paschall, (Eds), Starch: Chemistry and Technology, (pp. 183-247). Academic Press, Orlando, FL.

Fujii, M., Honda, K. and Fujita, H. (1973). Dilute solution of amylose in dimethylsulfoxide. Biopolymers, 12, 1177-1195.

Fuwa, H. (1982). Enzymic degradation of starch granules. Journal of Japanese Society of Slarch Science, 29, 99-106.

Fuwa, H., Nakajima, M., Hamada, A. and Glover, D. V. (1977). Comparative susceptibility to amylases of starches from different plant species and several single endosperm mutants and their double-mutant combinations with OPAQUE-2 inbred Oh43 maize. Cereal Chemistry, 54, 230-237.

Gallant, D. J., Bouchet, B. and Baldwin, P. M. (1997). Microscopy of starch: evidence of a new level of granule organization. Carbohydrate Polymers, 32, 177-191.

Gallant, D. J., Bouchet, B., Buléon A. and Pérez, S. (1992). Physical characteristics of starch granules and susceptibility to enzymatic degradation. European Journal of Clinical Nutrition, 46, S3-S16.

Galliard, T. (1987). Starch: properties and potential. (Vol 13): John Wiley, Chichester, U.K.

Galliard, T. and Bowler, P. (1987). Morphology and composition of starch. In T. Galliard, (Ed), Starch, Properties and Potential, (pp. 55-78) . John Wiley, Chichester, UK.

Genkina, N. K., Kiseleva, V. 1. and Noda, T. (2009). Comparative investigation on acid hydrolysis of sweet potato starches with different amylopectin chain-length. Starch, 61, $321-325$.

Gerard, C., Barron, C., Colonna, P. and Planchot, V. (2001). Amylose determination in genetically modified starches. Carbohydrate Polymers, 44, 19-27. 
Gerard, C., Colonna, P. Buleon, A. and Planchot, V. (2002). Order in maize mutant starches revealed by mild acid hydrolysis. Carbohydrate Polymers, 48, 131-141.

Gerard, C., Colonna, P., Bulcon, A. and Planchot, V. (2001). Amylolysis of maize mutant starches. Journal of Science and Food Agriculture, 81, 1281-1287.

Gernat, Ch., Radosta, S., Anger, H. and Damaschun, G. (1993). Crystalline parts of three different conformations detected in native and enzymatically degraded starches. Starch/Starke, 45, 309-314.

Gidley, M. J., and Bulpin, P. V. (1987). Crystallisation of malto-oligosaccharides as model of the crystalline forms of starch: Minimum chain-length requirement for the formation of double helices. Carbohydrate Research, 161, 291-300.

Gidley, M. J. and Bociek, S. M. (1985). Molecular organization in starches: A ${ }^{13}$ C.CP/MAS NMR study. Journal of the American Chemical Society, 107, 7040-7044.

Gillian, D. A. Sargent, J. G. and Schofield, J. D. (1981). Wheat starch granule proteins: the isolation and characterization of a salt-extractable protein from starch granules. Journal of the Science of Food and Agriculture, 32, 371-377.

Glaring, M. A., Koch, C. B. and Blennow, A. (2006). Genotype-specific spatial distribution of starch molecules in the starch granule: a combined CLSM and SEM approach, Biomacromolecules, 7, 2310-2320

Godet, M. C., Bizot, H. and Buléon, A. (1995). Crystallization of amylose-fatty acid complexes prepared with different amylose chain lengths. Carbohydrate Polymers, 27, 47-52.

Goesaert, H., Slade, L., Levine, H. and Delcour, J. A. (2009). Amylases and bread firming - an integrated view. Journal of Cereal Science, 50, 345-352.

Gomand, S. V., Lamberts, L., Visser, R. G. F. and Delcour, J. A. (2010). Physicochemical properties of potato and cassava starches and their mutants in relation to their structural properties. Food Hydrocolloids, 24, 424-433. 
Grabitske, A. H. and Slavin, J. L. (2009). Gastrointestinal effects of low digestible carbohydrates. Critical Review in Food Science and Nutrition, 49, 327-360.

Gray, G. M. (1992). Starch digestion and absorption in nonruminants. Journal of Nutrition, 122, $172-177$

Gray, J. A. and BeMiller, J. N. (2004). Development and utilization of reflectance confocal laser scanning microscopy to locate reaction sites in modified starch granules. Cereal Chemistry, 81, 278-286.

Greenwell, P. and Scofield, J. D. (1986). A starch granule protein associated with endosperm softness in wheat. Cereal Chemistry, 63, 379-380.

Greenwood, C. T. and Thompson, J. (1962). Physiochemical studies on starches. XXIV. Fractionation and characterization of starches of various plant origins. Journal of Chemical Society, 42, 222-229.

Gunaratne, A. and Corke, H. (2007). Gelatinizing, pasting and gelling properties of potato and amaranth starch mixtures. Cereal Chemistry, 84, 22-29.

Gunaratne, A. and Hoover, R. (2002). Effect of heat-moisture treatment on the structure and physicochemical properties of tuber and root starches. Carbohydrate Polymers, 40, 425437.

Gunaratne, A., Kong, X. and Corke, H. (2010). Functional properties and retrogradation of heatmoisture treated wheat and potato starches in the presence of hydroxypropyl $\beta$ cyclodextrin. Starch, 62,69-77.

Guraya, H. S., Kadan, R. S. and Champagne, E. T. (1997). Effect of rice starch-lipid complexes on in vitro digestibility, complexing index, and viscosity. Cereal Chemistry, 74, 561565.

Hagenimana, A. and Ding, X. (2005). A comparative study on pasting and hydration properties of native rice starches and their mixtures. Cereal Chemistry, 82, 72-76. 
Hall, D. M., and Sayre, J. G. (1970). A scanning electron microscope study of starches. II. Cereal starches. Textile Research Journal, 40, 256-266.

Han, X. Z. and Hamaker, B. R. (2002). Location of starch granule-associated proteins revealed by confocal laser scanning microscopy. Journal of Cereal Science, 35, 109-116.

Han, X. Z., Benmoussa, M., Gray, J. A., BeMiller, J. N. and Hamaker, B. R. (2005). Detection of proteins in starch granule channels. Cereal Chemistry, 82, 351-355.

Hanashiro, I., Tagawa, M., Shibahara, S., Iwata, K., Takeda, Y. (2002). Examination of molarbased distribution of A, B and C chains of amylopectin by fluorescent labelling with 2aminopyridine. Carbohydrate Research, 337, 1211-1215.

Hanes, C. S. (1937). The action of amylases in relation to the structure of starch and its metabolism in the plant. Parts I-III. New Phytologist, 36, 101-141.

Hedley, L., Bogracheva, T. Y. and Wang, T. L. (2002). A genetic approach to studying the morphology, structure and function of starch granules using pea as a model. Starch, 54, $235-242$.

Helbert, W. and Chanzy, H. (1994). Single-crystals of V-amylose complexed with n-butanol or n-pentanol structural features and properties. International Journal of Biological Macromolecules, 16, 207-213.

Hermans, P. H. and Weidinger, A. (1948). Quantitative X-ray investigations on the crystallinity of cellulose fibres. A background analysis. Journal of Applied Physics, 19, 419-506.

Hizukuri, S. (1985). Relationship between the distribution of the chain length of amylopectin and the crystalline structure of starch granules. Carbohydrate Research, 141, 295-306.

Hizukuri, S. (1986). Polymodal distribution of the chain lengths of amylopectins, and its significance. Carbohydrate Research, 147, 342-347.

Hizukuri, S., Kaneko, T. and Takeda, Y. (1983). Measurement of the chain length of amylopectin and its relevance to the origin or crystalline polymorphism of starch granules. Biochemica et Biophysica Acta, 760, 188-191. 
Hizukuri, S., Tabata, S. and Nikuni, Z. (1970). Studies on starch phosphate. Part 1. Estimation of glucose-6-phosphate residues in starch and the presence of other bound phosphate(s). Starch, 22, 338-343.

Hizukuri, S., Takeda, Y., Shitaozono, T., Abe, J., Ohtakara, A., Takeda, C. and Suzuki, A. (1988), Structure and Properties of Water Chestnut (Trapa natans L. var. bispinosa Makino) Starch. Starch, 40, 165-171

Hizukuri, S., Takeda, Y., Yasuda, M. and Suzuki, A. (1981). Multibranched nature of amylose and the action of de-branching enzymes. Carbohydrate Research, 94, 205-213.

Holm, J. and Bjorck, I. (1988). Effect of thermal processing of wheat on starch. II. Enzymic availability. Journal of Cereal Science, 8, 261-268.

Holm, J., Bjorck, I., Ostrowska, S., Eliasson, A. C., Asp, N. G., Larsson, K. and Undquist, I. (1983). Digestibility of amylose-lipid complexes in-vitro and in-vivo. Starch, 35, 294 297.

Hoover, R. (1998). Starch- Lipid Interactions. In R. H. Walter (Ed), Polysaccharide association structures in food, (pp. 227-256). Marcell Dekker, Inc. NY.

Hoover, R. (2000). Acid treated starches. Food Review International, 16, 369-392.

Hoover, R. (2001). Composition, molecular structure, and physico-chemical properties of tuber and root starches: a review. Carbohydrate Polymers, 45, 253-267.

Hoover, R. (2010). The impact of heat-moisture treatment on molecular structures and properties of starches isolated from different botanical sources. Critical Reviews in Food Science and Nutrition, 50, 1-13.

Hoover, R. and Hadziyev, D. (1981). Characterization of potato starch and its monoglyceride complexes. Starch, 33, 290-330.

Hoover, R. and Manuel, H. (1996). The effect of heat-moisture treatment on the structure and physicochemical properties of normal maize, waxy maize, dull waxy maize and amylomaize V starches. Journal of Cereal Science, 23, 153-162. 
Hoover, R. and Manuel, H. (1995). A comparative study of the physicochemical properties of starches from two lentil cultivars. Food Chemistry, 53, 275-284.

Hoover, R. and Ratnayake, W.S. (2004). Determination of total amylose content of starch. In Wrolstad et al. (Eds.), Handbook of Food Analytical Chemistry - Water, protein, enzymes, Lipids, and carbohydrates (pp. 689-691). Wiley-Interscience, Hoboken, NJ.

Hoover, R. and Sosulski, F. (1986). Effect of cross linking on functional properties of legume starches. Starch, 38, 149-155.

Hoover, R. and Sosulski, F. W. (1985). Studies on the functional characteristics and digestibility of starches from Phaseolus vulgaris biotypes. Starch, 37, 181-191.

Hoover, R. and Vasanthan, T. (1994c). The effect of annealing on the physicochemical properties of the wheat, oat, potato and lentil starches. Journal of Food Biochemistry, 17, 303-325.

Hoover, R. and Vasanthan, T. (1994a). Effect of heat-moisture treatment on the structure and physicochemical properties of cereal, legume and tuber starches. Carbohydrate Research, $252,33-53$.

Hoover, R, and Vasanthan, T. (1994b). The flow properties of native, heat-moisture treated and annealed starches from wheat, oat, potato and lentil. Journal of Food Biochemistry, 18, $67-82$.

Hoover, R., Hughes, T., Chung, H. J. and Liu, Q. (2010). Composition, molecular structure, properties and modification of pulse starches: A review. Food Research International, 43, 399-413.

Hoover, R., Swamidas, G. and Vasanthan, T. (1993). Studies on the physico- chemical properties of native, defatted, and heat-moisture treated pigeon pea (Cajanus cajan L.) starch. Carbohydrate Research, 246, 185-203.

Hoover, R., Vasanthan, T., Senanayake, N. J., and Martin, A. M. (1994). The effects of defatting and heat-moisture treatment on the retrogradation of starch gels from wheat, oat, potato and lentil. Carbohydrate Research, 26, 13-24. 
Hormdok, R. and Noomhorm, A. (2007). Hydrothermal treatments of rice starch for improvement of rice noodle quality, LWT - Food Science and Technology, 40, 17231731.

Huber, K. C. and BeMiller, J. N. (1997). Visualization of channels and cavities of corn and sorghum starch granules. Cereal Chemistry, 74, 537-541.

Huber, K. C. and BeMiller, J. N. (2000). Channels of maize and sorghum starch granules. Carbohydrate Polymers, 4I, 269-276.

Imam, S. H. (1989). A tightly bound $\mathrm{M}_{\mathrm{r}} 55,000$ polypeptide in cornstarch associated with the amylose portion of the granule. Journal of cereal Science, 9, 231-236.

Imberty, A. and Pérez, S. (1988). A revisit to the three dimensional structure of B-type starch. Biopolymers, 27, 308-325.

Imberty, A., Buléon, A., Tran, V. and Pérez, S. (1991). Recent advances in knowledge of starch structure. Starch, 43, 375-384.

Imberty, A., Chanzy, H., Pérez, S., Buléon, A. and Tran, V. (1988). The double helical nature of the crystalline part of A-starch. Journal of Molecular Biology, 201, 365-378.

Jacobasch, G., Dongowski, G., Schmiedl, D., and Schmehl, K. M. (2006). Hydrothermal treatment of Novelose 330 results in high yield of resistant starch type 3 with beneficial prebiotic properties and decreased secondary bile acid formation in rats. British Journal of Nutrition, 95, 1068-1074.

Jacobs, H. and Delcour, J. A. (1998). Hydrothermal modifications of granular starch, with retention of the granular structure: a review. Journal of Agricultural Food Chemistry, 46, 2895-2905.

Jacobs, H., Eerlingen, R. C., Rouseu, N., Colonna, P. and Delcour, J. A. (1998). Acid hydrolysis of native and annealed wheat, potato and pea starches-DSC melting features and chain length distributions of lintnerised starches. Carbohydrate Research, 308, 359-371

Jane, J. L. (2007). Structure of starch granules. Journal of Applied Glycoscience, 54, 31-36. 
Jane, J. L. and Robyt, J. F. (1984). Structure studies of amylose V complexes and retrogradaded amylose by action of alpha amylase, a new method for preparing amylodextrins. Carbohydrate Research, 132, 105-110.

Jane, J. L., Wong, K. S. and McPherson, A. E. (1997). Branch-structure difference in starches of A- and B-type X-ray patterns revealed by their Naegeli dextrins. Carbohydrate Research, 300,219-227.

Jane, J. L., Chen, Y. Y., Lee, L. F., McPherson, A. E., Wong, S., Radosavlievic, M. and Kasemsuwan, T. (1999). Effects of amylopectin branch chain length and amylose content on the gelatinization and pasting properties of starch. Cereal Chemistry, 76, 629-637.

Jane, J., Kasemsuwan, T., Chen, J. F. and Juliano, B. O. (1996). Phosphorus in rice and other starches. Cereal Foods World, 41, 827-832.

Jane, J., Kasemsuwan, T., Leas, S., Zobel, H. and Robyt, J. F. (1994). Anthology of starch granule morphology by scanning electron microscopy. Starch, 46, 121-129.

Jane, J. L., Xu, A., Radosavljevic, M. and Seib, P. A. (1992). Location of amylose in normal starch granules. I: susceptibility of amylose and amylopectin to cross-linking reagents. Cereal Chemistry, 69, 405- 409

Jane, J. L. and Shen, J. J. (1993). Internal structure of potato starch granule revealed by chemical gelatinization. Carbohydrate Research, 247, 279-290.

Jane, J. L. (2009). Structural features of starch granules II. In J. BeMiller, and R. Whistler (Eds), Starch:Chemistry and Technology, (pp 193-236). Academic Press NY:

Jayakody, L., Hoover, R., Liu, Q. and Donner, E. (2007). Studies on tuber starches. II. Molecular structure, composition and physicochemical properties of yam (Dioscorea $\mathrm{Sp}$.) starches grown in Sri Lanka. Carbohydrate Polymers, 69, 148-163.

Jenkins, D. J. A., Wolever, T. M. S., Collier, G. R., Ocana, A., Rao, A. V., Buckely, G., Lam, Y., Mayer, A., and Thompson, L. U. (1987). Metabolic effects of a low-glycemic-index diet. American Journal of Clinical and Nutrition, 46, 968-975. 
Jenkins, D. J. A., Wolever, T. M. S., Taylor, R. H., Barker, H., Fielder, H., Baldwin, J. M., Bowling, A. C., Newman, H. C., Jenkins, A. L. and Goff, D. V. (1981). Glycemic index of foods: a physiological basis of carbohydrate exchange. American Journal of Clinical Nutrition, 34, 362-366.

Jenkins, P. J. and Donald, A. M. (1995). The influence of amylose on starch granule structure. International Journal of Biological Macromolecules, 17, 315-321

Jenkins, P. J. and Donald, A. M. (1998). Gelatinisation of starch - a combined WAXS/SAXS/DSC and SANS study. Carbohydrate Research, 308, 133-147.

Jenkins, P. J., Cameron, R. E. and Donald, A. M. (1993). A universal feature in the structure of starch granules from different botanical sources. Starch, 45, 417-420.

John, M., Schmidt, J. and Kneifel, H. (1983). lodine-maltosaccharide complexes: relation between chain-length and colour. Carbohydrate Research, 119, 254-257.

Jouquand, C., Ducruet, V. and Le Bail, P. (2006). Formation of amylose complexes with C6aroma compounds in starch dispersions and its impact on retention. Food Chemistry, 96, 461470.

Juszczaka, L., Fortuna, T. and Krok, F. (2003). Non-contact Atomic Force Microscopy of Starch Granules Surface. Part I. Potato and Tapioca Starches. Starch, 55, 1-7.

Jyothi, A. N., Sajeev, M. S. and Sreekumar, J. N. (2010). Hydrothermal modifications of tropical tuber starches. 1. Effect of heat-moisture treatment on the physicochemical, theological and gelatinization characteristics. Starch, 62, 28-40.

Kainuma, K. and French, D. (1971). Nageli amylodextrin and its relationship to starch granule structure. 1. Preparation and properties of amylodextrins from various starch types. Biopolymers, 10, 1673-1680.

Kainuma, K. (1988). Structure and chemistry of starch granule. In P. K. Stumpf and E. E. Conn, (Eds), The biochemistry of plants, (pp. 141-180). Academic Press, NY. 
Kang, K. J., Kim, S., Lee, S. K. and Kim, S. K. (1997). Relationship between molecular structure of acid-hydrolyzed rice starch and retrogradation. Korean Journal of Food Science and Technology, 29, 876-881.

Karim, A. A., Norziah, M. H. and Seow, C. C. (2000). Methods for the study of starch retrogradation. Food Chemistry, 71, 9-36.

Karim, A. A., Toon, L. C., Lee, V. P., Ong, W. Y., Fazilah, A. and Noda, T. (2007). Effects of phosphorus contents on the gelatinization and retrogradation of potato starch. Journal of Food Science, 72, C132-138.

Karkalas, J., Ma, S., Morrison, W. and Pethrick, R. A. (1995). Some factors determining the thermal properties of amylose inclusion complexes with fatty acids. Carbohydrate Research, 268, 233-247.

Karkalas, J., Ma, S., Morrison, W. R. and Pethrick, R.A. (1995). Some factors determining the thermal properties of amylose inclusion complexes with fatty acids. Carbohydrate Research, 268, 233-247.

Kasemsuwan, T. and Jane, J. (1994). Location of amylose in normal starch granules. II. Location of phosphodiester cross-linking revealed by phosphorous-31 nuclear magnetic resonance. Cereal Chemistry, 71, 282-287

Kawabata, A., Takase, N., Miyoshi, T., Sawayama, S., Kimura, T. and Kudo, K. (1994). Microscopic observation and X-ray diffractometry of heat-moisture treated starch granules. Starch, 48, 463-469.

Khunae, P., Tran, T., and Sirivongpaisal, P. (2007). Effect of heat-moisture treatment on structural and thermal properties of rice starches differing in amylose content. Starch, 59, 593-599.

Kim, H. and Huber, K. (2008). Channels within soft wheat starch A- and B-type granules. Journal of Cereal Science, 48, 159-172.

Kim, R. E. and Ahn, S. Y. (1996). Gelling properties of acid-modified red bean starch gels. Agriculture Chemistry and Biotechnology, 39, 49-53. 
Kitamura, S. and Kuge, T. (1989), A differential scanning calorimetric study of the conformational transitions of schizophyllan in mixture of water and dimethylsulfoxide. Biopolymers, 28, 639-654.

Klucinec, J. D. and Thompson, D. B. (1998). Fractionation of high amylose maize starches by differential alcohol precipitation and chromatography of the fractions. Cereal Chemistry, $75,887-896$.

Knutson, C. A., Khoo, U., Cluskey, J. E. and Inglett, G. E. (1982). Variation in enzyme digestibility and gelatinization behaviour of corn starch granule fractions. Cereal Chemistry, 59, 512-515.

Kotting, O., Pusch, K., Tiessen, A., Geigenberger, P., Steup, M. and Ritte, G. (2005). Identification of a novel enzyme required for starch metabolism in Arabidopsis leaves. The phosphoglucan, water dikinase. Plant Physiology, 137, 242-252.

Kozlov, S. S., Noda, T., Bertoft, E. and Yuryev, V. P. (2006). Structure of starches exctracted from near-isogenic wheat lines. Part I. Effect of different GBSS I combinations. Journal of Thermal Analysis and Calorimetry, 86, 291-301.

Kozlov, S. S., Blennow, A., Krivandin, A. V. and Yuryev, V. P. (2007). Structural and thermodynamic properties of starches extracted from GBBS and GWD suppressed potato lines. International Journal of Biological Macromolecules, 40, 449-460.

Kubelka, P. and Munk, F. (1931). An article on optics of paint layers. Physik, 12, 593-601.

Kugimiya, M. and Donovan, J. W. (1981). Calorimetric determination of the amylose content of starches based on the formation and melting of the amylose-lysolecithin complex. Journal of Food Science, 46, 765 .

Kulp, K. and Lorenz, K. (1981). Heat-moisture treatment of starches. I. Physicochemical properties. Cereal Chemistry, 58, 46-48.

Kurahashi, Y. and Yoshino, Z. (2000). HMT starch: Its production, properties, and uses (in Japanese), Oyo Toshitsu Kagaku 47, 125-132. 
Kurakake, M., Tachibana, Y., Masaki, K. and Komaki, T. (1996). Adsorption of alpha-amylase on heat-moisture treated starch. Journal of Cereal Science, 23, 163-168.

Kweon, M., Haynes, L., Slade, L. and Levine, H. (2000). The effect of heat and moisture treatments on enzyme digestibility of AeWx, Aewx and aeWx corn starches. Journal of Thermal Analytical Calorimetry, 59, 571-586.

Langton, M. and Hermasson, A. M. (1989). Microstructural changes in wheat starch dispersions during heating and cooling. Food Microstructure, 8, 29-39.

Lansky, S., Kooi, M. and Schoch, T. J. (1949). Properties of the fractions and linear subfractions from various starches. Journal of the American Chemical Society, 71, 4066-4075.

Lawal, O. S. (2005). Studies on the hydrothermal modifications of new cocoyam (Xanthosoma sagittifolium) starch. International Journal of Biological Macromolecules, 37, 268-277.

Lawal, O. S. and Adebowale, K. O. (2005). An assessment of changes in thermal and physicochemical parameters of jack bean (Canavalia ensiformis) starch following hydrothermal modifications. European Food Research and Technology, 221, 631-638.

Le Corre, D., Bras, J. and Dufresne, A. (2010). Starch Nanoparticles: A Review Biomacromolecules, 11, 1139-1153.

Leach, W., McCowen, D. and Schoch, T. J. (1959), Structure of the starch granule. I. Swelling and solubility patterns of various starches. Cereal Chemistry, 36, 534-544.

Lee, S. H. and BeMiller, J. N. (2008). Lysophosphatidylcholine identified as channel-associated phospholipid of maize starch granules. Cereal Chemistry, 85, 776-779.

Levine, H. and Slade, L. (1988). Non-equilibrium behaviour of small carbohydrate-water systems. Pure Applied Chemistry, 60, 1841.

Levine, H. and Slade, L. (1989). Influence of glassy and rubbery states on the thermal, mechanical, and structural properties of doughs and baked products. In H. Fairidi, J. M Faubion, (Eds), Dough rheology and baked product texture, (pp 157-300). Van Nostrand Reinhold, NY. 
Li, J. H., Vasanthan, T., Rossnagel, B. and Hoover, R. (2001). Starch from hull-less barley: I. Granule morphology, composition, and amylopectin structure. Food Chemistry, 74, 395405 .

Li, J-Y. and Yeh, An-I. (2001). Relationships between thermal, rheological characteristics and swelling power for various starches. Journal of Food Engineering, 50, 141-148.

Lim, C. C., Ferguson, R. L. and Tannock G. W. (2005). Dietary fibres as "prebiotics": Implications for colorectal cancer. Molecular Nutrition and Food Research, 49, 609 619.

Lim, S. T., Chang, E. H. and Chung, H. J. (2001). Thermal transition characteristics of heatmoisture treated maize and potato starches. Carbohydrate Polymers, 46, 107-115.

Lim, S., Kasemsuwan, T. and Jane, J. (1994). Characterization of phosphorus in starch by $31 \mathrm{P}-$ nuclear magnetic resonance spectroscopy. Cereal Chemistry, 71, 488-493.

Lin, P.-Y. and Czuchajowska, Z. (1998). Role of Phosphorus in Viscosity, Gelatinization, and Retrogradation of Starch. Cereal Chemistry, 75, 705-709.

Lindeboom, N., Chang, P. R. and Tylera, R. T (2004). Analytical, biochemical and physicochemical aspects of starch granule size, with emphasis on small granule starches: A review. Starch, 56, 89-99.

Lineback, D. R. (1986). Current concepts of starch structure and its impact on properties. Journal of Japanese Society of Starch Science, 33, 80-88.

Lineback, D. R. (1984). The starch granule organisation and properties. Bakers Digest, 13, 1621.

Lineback, D. R. and Rasper, V. F. (1988). Wheat carbohydrates. In Y. Pomeranz, (Ed), Wheat, Chemistry and Technology, (pp. 277-372). AACC, St Paul, MN.

Liu, J. and Zhoa, S. (1990). Scanning Electron Microscope Study on Gelatinization of Starch Granules in Excess Water. Starch, 42, 96-98. 
Liu, Q., Charlet, G., Yelle, S. and Arul, J. (2002). Phase transition in potato starch-water system. I. Starch gelatinisation at high moisture level. Food Research International, 35, $397-407$.

Liu, Q., Gu, Z., Donner, E., Tetlow, L. and Emes, M. (2007). Investigation of digestibility in vitro and physicochemical properties of A and B-type starch from soft and hard wheat flour. Cereal Chemistry, 84, 15-21.

Loisel, C., Maache-Rezzoug, Z., Esneault, C. and Doublier, J. L. (2006). Effect of hydrothermal treatment on the physical and rheological properties of maize starches. Journal of Food Engineering, 71, 45-54.

Lopez, E. C., Rolee, A. and Le Meste, M. (2004). Study of starch granules swelling by the blue dextran method and by microscopy. Starch, 56, 576-581.

Lopez-Rubio, A., Flanagan, B. M., Gilbert, E. P., and Gidley, M. J. (2008a). A novel approach for calculating starch crystallinity and its correlation with double helical content: A combined XRD and NMR study. Biopolymers, 89, 761-768.

Lopez-Rubio, A., Flanagan, B. M., Shrestha, A. K., Gidley, M. J. and Gilbert, E. P. (2008b). Molecular rearrangement of starch during in vitro digestion: towards a better understanding of enzyme resistant starch formation in processed starch. Biomacromolecules, 9, 19511958.

Lorberth, R., Ritte, G., Willmitzer, L. and Kossmann, J. (1998). Inhibition of a starch-granulebound protein leads to modified starch and repression of cold sweetening. Nature Biotechnology, 16, 473-477.

Lorenz, K. and Kulp, K. (1981). Heat-moisture treatment of starches. II. Functional properties and baking potential. Cereal Chemistry, 58, 49-52.

Lorenz, K., and Kulp, K. (1982). Cereal and root starch modification by heat-moisture treatment. 1. physicochemical properties. Starch, 34, 50-54. 
Lu, S., Chen, C.Y. and Lii, C.Y. (1996). Gel chromatography fractionation and thermal characterization of rice starch affected by hydrothermal treatment. Cereal Chemistry, 73, 5-11.

Lund, D. (1984). Influence of time, moisture, ingredients, and processing conditions on starch gelatinization. Critical Review in Food Science and Nutrition, 20, 249-273.

Luo, F.-X., Huang, Q., Fu, X., Zhang, L. X. and Yu, S.-J. (2009). Preparation and characterisation of crosslinked waxy potato starch. Food Chemistry, 115, 563-568

MacGregor, E. A., Janeček, Š. and Svensson, B. (2001). Relationship of sequence and structure to specificity in the $\alpha$-amylase family of enzymes. Biochimica et Biophysica Acta, 1546, $1-20$.

Marchant, J. L. and Blanshard, J. M. V. (1978). Studies of the dynamics of the gelatinization of starch granules employing a small angle light scattering system, Starch/Stärke, 30, 257264.

Matveev, Y. L., Elankin, N. I., Kalistratova, E. N., Danilenko, A. N., Niemann, C. and Yuryev, V. P. (1998). Estimation of contributions of hydration and glass transition to heat capacity changes during melting of native starches at excess water. Starch, 50, 141-147.

Matveev, Y. I., van Soest, J. J. G., Nieman, C., Wasserman, L. A., Protserov, V. and Ezernitskaja, M. and. Yuryev, V. P. (2001). The relationship between thermodynamic and structural properties of low and high amylose maize starches. Carbohydrate Polymers, 44, 151-160.

McPherson A. E. and Jane, J. (1999). Comparison of waxy potato with other root and tuber starches. Carbohydrate Polymers, 40, 57-70.

Mercier, C., Charbonnier, R. Grebaut, J. and De La Gueriviere, J. F. (1980). Formation of amylose lipid complexes by twin-screw extrusion cooking of manioc starch. Cereal chemistry, 57, 4-9. 
Mikkelsen, R. Baunsgaard, L., Blennow, A. (2004). Functional characterization of alphaglucan,water dikinase, the starch phosphorylating enzyme. Biochemistry Journal, 377, $525-532$.

Miles, M. J., Morris, V. J., Oxford P. D. and Ring, S. G. (1985). The roles of amylose and amylopectin in the gelation and retrogradation of starch. Carbohydrafe Research, 135, $271-281$.

Mishra, S and Raj, T. (2006). Morphology and functional properties of corn, potato and tapioca starches. Food Hydrocolloids, 20, 557-566.

Miyoshi, E. (2002). Effect of heat-moisture treatment and lipids on gelatinization and retrogradation of maize and potato starches. Cereal Chemistry, 79, 72-77.

Moorthy, S.N. (1999). Effect of steam pressure treatment on the physicochemical properties of dioscorea starches. Journal of Agricultural and Food Chemistry, 47, 1695-1699.

Morell, M. K., Samuel, M. S. and O'Shea, M. G. (1998). Analysis of starch structure using fluorophore-assisted carbohydrate electrophoresis. Electrophoresis, 19, 2603-2611.

Morrison, W. R. (1981). Starch lipids: A reappraisal. Starch, 33, 408-410.

Morrison, W. R. (1985). Extraction of lipids from cereal starches with hot aqueous alcohols. Starch, 37, 83-87.

Morrison, W. R. (1988). Lipids in cereal starches: A review. Journal of Cereal Science, 8, 1-15

Morrison, W. R. and Karkalas, J. (1990). Starch. In P.M. Dey, (Ed), Methods in plant biochemistry: carbohydrates, (pp. 252-323). Academic Press, London, UK.

Morrison, W. R., Milligan, T. P. and Azudin, M. N. (1984). A relationship between the amylose and lipid contents of starches from diploid cereals. Journal of Cereal Science, 2, 257271 .

Morrison, W. R., Tester, R. F., Snape, C. E., Law, R. and Gidley, M. J. (1993a). Swelling and gelatinization of cereal starches: IV. Some effects of lipid complexed amylose and free amylose in waxy and normal barley starches. Cereal Chemistry. 70, 385-391 
Morrison, W. R. (1964). A fast simple and reliable method for the micro determination of phosphorus in biological material. Analytical Biochemistry, 7, 218-224.

Morrison, W. R., Law, R. V. and Snape, C. E. (1993b). Evidence for inclusion complexes of lipids with V-amylose in maize, rice and oat starches. Journal of Cereal Science, 18, 107-109.

Mua, J. P. and Jackson, D. S. (1997). Fine structure of corn amylose and amylopectin fractions with various molecular weights. Journal of Agricultural and Food Chemistry, 45, 38403847 .

Mu-Forster, C. and Wasserman, B. P. (1988). Surface localisation of zein storage proteins in starch granules from maize endosperm. Proteolytic removal by thermolysin and in-vitro cross-linking of granule associated polypeptides. Plant Physiology, 116, 1563-1571.

Mu-Forster, C., Huang, R., Powers, J. R., Harriman, R.W., Knight, M., Singletary, G. W., Keeling P. L. and Wasserman, B. P. (1996). Physical association of starch biosynthetic enzymes with starch granules of maize endosperm. Granule-associated forms of starch synthase I and starch branching enzyme II. Plant Physiology, 111, 821-829.

Mutungi, C., Rost, F., Onyango, C., Jaros, D. and Rohm, H. (2009). Crystallinity, thermal and morphological characteristics of resistant starch type III produced by hydrothermal treatment of debranched cassava starch. Starch, 61, 634-645.

Myers, A. M., Morell, M. K., James, M. G. and Ball, S. G. (2000). Recent progress toward understanding biosynthesis of the amylopectin crystal. Plant Physiology, 122, 989-997

Nakazawa Y. and Wang, Y. J. (2004). Effect of annealing on starch-palmitic acid interaction. Carbohydrate Polymers, 57,327-335.

Nakazawa, Y. and Wang, J-J. (2003). Acid hydrolysis of native and annealed starches and branch-structure of their Naegeli dextrins. Carbohydrate Research, 338, 2871-2882.

Nara, S., Mori, A. and Komiya, T. (1978). Starch, 30, 111-114.

Nguyen, Q. T., Aptel, P and Neel, J. (1976). Biopolymers, 15, 2097 
Noda, T., Kimura, T., Otani, M., Ideta, O., Shimada T., Saito, A. and Suda, I. (2002). Physicochemical properties of amylose-free starch from transgenic sweet potato. Carbohydrate Polymers, 49, 253-260.

Noda, T., Takigawa, S., Matsuura-Endo, C., Kim, S.-J., Hashimoto, N., Yamauchi, H., Hanashiro, I. and Takeda, Y. (2005). Physicochemical properties and amylopectin structures of large, small, and extremely small potato starch granules. Carbohydrate Polymers, 60, 245-251.

O'Brien, S. O. and Wang, Y. J. (2008). Susceptibility of annealed starches to hydrolysis by $\alpha$ amylase and glucoamylase. Carbohydrate Polymers, 72, 597-607.

O'Shea, M. G., Samuel, M. S., Konik, C. M. and Morell, M. K. (1998). Fluorophore-assisted carbohydrate electrophoresis (FACE) of oligosaccharides - Efficiency of labelling and high-resolution separation. Carbohydrate Research, 307, 1-12

Oates, C. G. (1990). Evidence for protein crosslinks in mung-bean starch. In G. O. Phillips, D. J. Wedlock, and P. A. Williams, (Eds). Gums and Stabilisers for the Food Industry, (pp 203-206). IRL Press, Oxford, UK.

Oates, C. G. (1997). Towards an understanding of starch granule structure and hydrolysis. Trends in Food Science and Technology, 82, 375-382.

Olayinka, O., Adebowale, K. O. and Olu-Owolabi, B. I. (2008). Effect of heat-moisture treatment on physicochemical properties of white sorghum starch. Food Hydrocolloids, $22,225-230$.

Osunsam, A. T., Akingbala, J. O., and Oguntimein, G. B. (1989). Effect of storage on starch content and modification of cassava starch. Starch, 41, 54-57.

Paris, M., Bizot, H., Emery, J., Buzare, J. Y. and Buleon, A. (2001). NMR local range investigations in amorphous starchy substrates I. Structural heterogeneity probed by ${ }^{13} \mathrm{C}$ CP-MAS NMR. International Journal of Biological Macromolecules, 29, 127-136. 
Peat, S., Whelan,W. J., Thomas,G. J. (1952). Evidence of multiple branching in waxy maize starch, (pp. 4546-4548). Journal of the Chemical Society Chemical Communications, London.

Peng, M., Gao, M., Abdel-Aal, E. S. M., Hucl, P. and Chibbar, R. N. (1999). Separation and characterization of A- and B-type starch granules in wheat endosperm. Cereal Chemistry, 76, 375-379.

Perera, C. and Hoover, R. (1998). The reactivity of porcine pancreatic $\alpha$-amylase towards native, defatted and heat-moisture treated potato starches before and after hydroxypropylation. Starch, 50, 206-213.

Pérez, S. and Bertoft, E., (2010). The molecular structures of starch components and their contribution to the architecture of starch granules: A comprehensive review. Starch, 62, 389-420.

Peroni, F. H. G., Rocha, T. S. and Franco, C. M. L. (2006). Some Structural and Physicochemical Characteristics of Tuber and Root Starches. Food Science and Technology International, 12, 505-513.

Perry, P. A. and. Donald, A. M. (2002). The effect of sugars on the gelatinisation of starch. Carbohydrate Polymers, 49, 155-165.

Planchot V., Colonna, P., Gallant D. J., and Bouchet, B. (1995). Extensive degradation of native starch granules by á-amylase from Aspergillus fumigatus. Journal of Cereal Science, 21 , 163-171.

Planchot, V., Colonna, P., Buleon, A. and Gallent, D. (1997). Amylolysis of starch granules and $\alpha$-glucan crystallites. In R. J. Frazier, A. M. Donald and P. Richmond, (Eds), Starch structure and functionality, (pp. 141-152). Royal Society of Chemistry, Cambridge, UK.

Prentice, R. D. and Stark, J. R. (1992). Granule residues and "ghosts" remaining after heating Atype barley-starch granules in water. Carbohydrate Research, 227, 121-130. 
Purwani, E. Y., Widaningrum, R., Thahir, and Muslich (2006). Effect of heat- moisture treatment of sago starch on its noodle quality. Indonesian Journal of Agricultural Science, 7,8-14.

Qi, X., Tester, R. F, Snape, C. E. and Ansell, R. (2003). Molecular basis of the gelatinisation and swelling characteristics of waxy rice starches grown in the same location during the same season. Joumal of Cereal Science, 37, 363-376.

Qi, X., Tester, R. F., Snape, C. E., Yuryev, V., Wasserman, L. A. and Ansell, R. (2004). Molecular basis of the gelatinisation and swelling characteristics of waxy barley starches grown in the same location during the same season. Part II. Crystallinity and gelatinisation characteristics. Journal of Cereal Science, 39, 57-66.

Quigley, T. A., Kelly C. T., Doyle E. M. and Fogarty W. M. (1998). Patterns of raw starch digestion by the glucoamylase of Cladosporium gossypiicola ATCC 38026. Process Biochemistry, 33, 677-681

Rahman, S., Kosar-Hashemi, B., Samuel, M. S., Hill, A., Abbot, D. C., Skerritt, J. H., Preiss, J., Appels, R. and Morell, M. K. (1995). The major proteins of wheat endosperm starch granules. Australian Journal of Plant Physiology, 22, 793 -803.

Rao, V. S. R. and Foster, J. F. (1963). Studies of the conformation of amylose in solution. Biopolymers, l, 527-544.

Raphaelides, S. and Karkalas, J. (1988). Thermal dissociation of amylose-fatty acid complexes. Carbohydrate Research, 172, 65-82.

Rappenecker, G. and Zugenmaier, P. (1981). Detailed refinement of the crystal structure of Vhamylose. Carbohydrate Research, 89, 11-19.

Ratnayake W. S., Hoover, R., Shahidi, F., Perera, C. and Jane, J. (2001). Composition, molecular structure and physicochemical properties of starches from four field pea (Pisum sativum L.) cultivars. Food Chemistry, 74, 189-202.

Ratnayake, W. S., Hoover, R. and Warkentin, T. (2002). Pea starch: composition, structure and properties - A Review. Starch, 54, 217-234. 
Rendleman, J. A. J. (2003). The reaction of starch with iodine vapor. Determination of iodideion content of starch-iodine complexes. Carbolydrate Polymers, 51, 191-202.

Rickard, J. E., Asaoka, M. and Blanshard, J. M. V. (1991). Review of the physicochemical properties of cassava starch. Tropical Science, 31, 189-207.

Ridout, M. J., Gunning, A. P., Parker, M. L., Wilson, R. H. and Morris, V. J. (2002). Using AFM to image the internal structure of starch granules. Carbohydrate Polymers, 50, 123-132.

Riisom, T., Krog, N., and Eriksen, J. (1984). Amylose complexing capacities of cis- and transunsaturated monoglycerides in relation to their functionality in bread. Journal of Cereal Science, 2, 105-118.

Ring, S. G., Gee, M. J., Whittam, M., Orford P. and Johnson, I. T. (1988). Resistant starch: its chemical form in foodstuffs and effect on digestibility in vitro. Food Chemistry, 28, 97109.

Ring, S. G., Collona, P., Panson, K. J., Kalicheversky, M. T., Miles, M. J., Morris, V. J. and Oxford, P. D. (1987). The gelation and crystallization of amylopectin. Carbohydrate Research, 162, 277-293.

Ring, S, G., L'Anson, K. J, and Morris, V. J. (1985). Static and dynamic light scattering studies of amylose solutions. Macromolecule, 18, 182-188.

Ritte, G., Heydenreich, M., Mahlow, S., Haebel, S., Kötting, O. and Steup, M. (2006). Phosphorylation of C6- and C3-positions of glucosyl residues in starch is catalyzed by distinct dikinases. FEBS Letters, 580, 4872-4876.

Ritte, G., Lloyd, J., Eckermann, N., Rottmann, A., Kossmann, J. and Steup, M. (2002). The starch-related R1 protein is an $\alpha$-glucan, water dikinase. Plant Biology, 99, 7166-7171

Roach, R. R. and Hoseney, R. C. (1995). Effect of certain surfactants on the swelling, solubility and amylograph consistency of starch. Cereal Chemistry, 72, 571-577. 
Robin, J. P., Mercier, C., Charbonnière, R. and Guilbot, A. (1974). Lintnerized starches. Gelfiltration and enzymatic studies of insoluble residues from prolonged acid treatment of potato starch. Cereal Chemistry, 51,389-406.

Robin, J. P., Mercier, C., Duprat, F., Charbonnière, R. and Guilbot, A. (1975). Lintnerized starches. Chromatographic and enzymatic studies of insoluble residues from acid hydrolysis of various cereal starches, particularly, waxy maize starch. Starch, 27, 36-45

Robyt, J. F and French, D. (1970). The action pattern of porcine pancreatic $\alpha$-amylase in relationship to the substrate binding site of the enzyme. Journal of Biological Chemistry, $245,3917-3927$.

Robyt, J. F. (2009). Enzymes and their action on starch. In J. BeMiller, and R. Whistler (Eds), Starch:Chemistry and Technology, (pp 238-284). Academic Press, NY:

Rundle R. E. (1947). The Configuration of Starch in the Starch-Iodine Complex. V. Fourier Projections from X-Ray Diagrams. Journal of American Chemical Society, 69, 17691772 .

Rundle, R. E. and French, D. (1943). The configuration of starch in the starch-iodine complex. III. X-ray diffraction studies of the starch-iodine complex. Journal of the American Chemical Society, 65, 1707-1710.

Rye, C. S and Withers, S. G. (2000). Glycosidase mechanisms. Current Opinion in Chemical Biology, 4, 573-580.

Saenger, W. (1984). The structure of the blue starch-iodine complex. Naturwissenschaften, 71 , 31-36.

Saibene D. and Seetharaman, K. (2006). Segmental mobility of polymers in starch granules at low moisture contents. Carbohydrate Polymers, 64, 539-547.

Saibene, D. and Seetharaman, K. (2010). Amylose involvement in the amylopectin clusters of potato starch granules. Carbohydrate Polymers, 82, 376-383. 
Saibene, D., Zobel, H. F., Thompson, D. B. and Seetharaman, K. (2008). Iodine binding by granular corn and potato starch: Evidence for differential location of amylose. Starch, $60,165-173$.

Sair, L. (1967). Heat-moisture treatment of starch. Cereal Chemistry, 44, 8-26

Saitô, H., Yamada, J., Yukumoto, T., Yajima, H. and Endo, R. (1991). Conformational stability of $\mathrm{V}$-amyloses and their hydration induced conversion to B-type form as studied by highresolution solid-state C13 NMR-spectroscopy. Bulletin of the Chemical Society of Japan, $64,3528-3537$.

Sajilata, M. G. Singhal, R. S. and Kulkarni, P. R. (2006). Resistant starch-A review. Comprehensive Reviews in Food Science and Food Safety, 5, 1-17.

Sakonidou, E. P., Karapantsios, T. D. and Raphaelides, S. N. (2003). Mass transfer limitations during starch gelatinization. Carbohydrate Polymers, 53, 53-59.

Sang, Y., and Seib, P. A. (2006). Resistant starchesfrom amylosemutants of corn by simultaneous heat-moisture treatment and phosphorylation. Carbohydrate Polymers, 63, 167-175.

Sasaki, T. and Matsuki, J. (1998). Effect of wheat starch structure on swelling power. Cereal Chemistry, 75, 525-529.

Satin, M. (1998). Functional properties of starches. In Spolight tropical starch misses market. AGSIreport, Agriculture21, FAO-Magazine, $11 \mathrm{p}$.

Satin, M. (2000). Functional properties of starches. In: International Symposium on Tropical Root and Tuber Crops, Thiruvananthapuran (Trivandrum).

Schoch, T. J. (1942). Non-carbohydrate substance in the cereal starches. Journal of American Chemical Society, 64, 2954-2956.

Schoch, T. J. and Maywald, E. C. (1956). Microscopic examination of modified starches. Analytical Chemistry, 28, 382-387 
Schofield, J. D. and Greenwell, P. (1987). Wheat starch granules and their technological significance. In I. D. Morton, (Ed), Cereals in a European context, (pp. 407-420). Elis Horwood/VCH, England/Weinheim.

Schwall, G. P., Safford, R., Westcott, R. J., Jeffcoat, R., Tayal, A., Shi, Y. C., Gidley, M. J. and Jobling, S. A. (2000). Production of very-high-amylose potato starch by inhibition of SBE A and B. Nature of Biotechnology, 18,551-554

Seguchi, M. and Yamada, Y. (1989). Study of proteins extracted from the surface of wheat starch granules with sodium dodecyl sulphate. Cereal Chemistry, 66, 193-196.

Seneviratne, H. D. and Biliaderis, C. G. (1991). Action of $\alpha$-amylases on amylose-lipid complex superstructure. Journal of Cereal science, 13, 129-141.

Senior, M. B. and Hamori, E. (1973). Investigation of the effect of amylose/ iodine complexation on the conformation of amylose in aqueous solution. Biopolymers, 12, 6578.

Sevenou, O., Hill, S. E., Farhat, I. A., Mitchell, J. R. (2002). Organization of the external region of the starch granule as determined by infrared spectroscopy. International Journal of Biological Macromolecules, 31, 79-85.

Shannon, J. C. and Garwood, D. L. (1984). Genetics and physiology of starch development. In R. L. Whistler, J. N. Bemiller, and E. F. Paschall, (Eds), Starch: Chemistry and Technology, (pp. 25-86). Academic Press, Orlando, FL.

Shannon, J. C., Pien, F. M., Cao, H. and Liu, K. C. (1998). Brittle-1, an adenylate translocator, facilitates transfer of extraplastidial synthesized ADP-glucose into amyloplasts of maize endosperms. Plant Physiology, 117, 1235-1252.

Shewry, P. R. (2003). Tuber storage proteins. Annals of Botany, 91, 755-769.

Shi, Y.-C. and Seib, P. A. (1992). The structure of four waxy starches related to gelatinization and retrogradation. Carbohydrate Research, 227, 131-145. 
Shih, F., King, J., Daigle, K., An, H. J., and Ali, R. (2007). Physicochemical properties of rice starch modified by hydrothermal treatments. Cereal Chemistry, 84, 527-553.

Shin, S. I., Choi, H. J., Chung, K. M., Hamaker, B. R., Park, K. H., and Moon, T. W. (2004). Slowly digestible starch from debranched waxy sorghum starch: Preparation and properties. Cereal Chemistry, 81, 404-408.

Shin, S. I., Kim, H. J., Ha, H. J., Lee, S. H., and Moon, T. W. (2005). Effect of hydrothermal treatment on formation and structural characteristics of slowly digestible non-pasted granular sweet potato starch. Starch, 57, 421-430.

Shogren, R. (2007). Effect of Orientation on the Physical Properties of Potato Amylose and High-Amylose Corn Starch Films. Biomacromolecules, 8, 3641-3645.

Shogren, R. L., Fanta, G. F. and Felker, F. C. (2006). X-ray diffraction study of crystal transformations in spherulitic amylose/lipid complexes from jet-cooked starch. Carbohydrate Polymers, 64, 444-451.

Singh, J. and Singh, N. (2001). Studies on the morphological, thermal and rheological properties of starch separated from some Indian potato cultivars. Food Chemistry 75, 67-77.

Singh, J. Dartois, A. and Kaur, L. (2010). Strach digestibility in food matrix: a review. Trends in Food Science and Technology, 21, 168-180.

Singh, J., McCarthy, O. J. and Singh, H. (2006). Physico-chemical and morphological characteristics of New Zealand Taewa (Maori potato) starches. Carbohydrate Polymers, 64, 569-581.

Singh, J., Singh, N. and Saxena, S. K. (2002). Effect of fatty acids on the rheological properties of corn and potato starch. Journal of Food Engineering, 52, 9-16.

Singh, N., Singh, J., Kaur, L., Sodhi, N. S. and Gill, B. S. (2003). Morphological, thermal and rheological properties of starches from different botanical sources. Food Chemistry, 81, 219-231. 
Singh, S., Raina, C. S., Bawa, A. S., and Saxena, D. C. (2005). Effect of heat- moisture treatment and acid modification on rheological, textural, and differential scanning calorimetry characteristics of sweet potato starch. Journal of Food Science, 70, E373E378.

Slade, L. and Levine, H. (1988). Non-equilibrium melting of native granular starch. Part I. Temperature location of the glass transition associated with gelatinization of A-type cereal starches. Carbohydrate Polymers, 8, 183-208.

Slaughter, S. L., Ellis, P. R. and Butterworth, P. J. (2001). An investigation of the action of porcine pancreatic $\alpha$-amylase on native and gelatinised starches. Biochimica et Biophysica Acta, 1525, 29-36.

Smith, O. (1987). Effect of cultural and environmental conditions on potatoes for processing. In Talburt, William, F, and Smith, Ora, (Eds), Potato processing, Van Nostrand, Reinhold, NY.

Sopade, P. A. (1990). Starch gelatinisation and retrogradation in food systems with special references to baked foods. In A. J. Rayar, B. K. Kaigama, J. O. Olukosi and A. B. Anaso (Eds), Wheat in Nigeria: Production, processing and utilization (pp. 251-261).

South, J. B. Morrison, W. R. and Nelson, O. E. (1991). A relationship between the amylose and lipid contents of starches from various mutants for amylose content in maize. Journal of Cereal Science, 14, 267-278.

Srichuwong, S, Sunarti, T. C, Mishima, T., Isono, N. and Hisamatsu, M. (2005). Starches from different botanical sources. II: Contribution of starch structure to swelling and pasting properties. Carbohydrate Polymers, 62,25-34.

Srichuwong, S., Sunarti, T. C. Mishima, T., Isono, N and Hismatsu, M. (2005). Starches from different botanical sources. I: Contribution of amylopectin fine structure to thermal properties and enzyme digestibility. Carbohydrate Polymers, 60, 529-538.

Sterling, C. (1960). Crystallinity of potato starch. Starch, 12, 182.

Sterling, C. (1973). Pore size in potato starch. Starch, 25, 115-118. 
Stevens, D. J. and Elton, G. A. H. (1971). Thermal properties of the starch/water system. Part I. Measurement of heat of gelatinisation by differential scanning calorimetry. Starch, 23, 8 11.

Stoddard, F. L. (1999). Survey of starch particle-size distribution in wheat and related species, Cereal Chemistry, 76, 145-149.

Stute, R. 1992). Hydrothermal modification of starches: The difference between annealing and heat-moisture-treatment. Starch, 44, 205-214

Sujka, M. and Jamroz, J. (2007). Starch granule porosity and its changes by means of amylolysis. International Agrophysics, 21, 107-113.

Sujka, M. and Jamroz, J. (2009). a-Amylolysis of native potato and corn starches - SEM, AFM, nitrogen and iodine sorption investigations. LWT - Food Science and Technology, 42, 1219-1224.

Suryanarayana, C. and Norton, M. G. (1998). Practical aspects of X-ray diffraction. In: C. Suryanarayana and M.G. Norton, Editors, X-ray diffraction: A practical approach, Plenum Press, NY (1998), pp. 63-94.

Svegmark, K. and Hermansson, A. M. (1993). Microstructure and rheological properties of composites of potato starch granules and amylose: a comparison of observed and predicted structure. Food Structure, 12, 181-193.

Svegmark, K., Helmersson, K., Nilsson, G., Nilsson, P. O., Anderson, R., and Svenson, E. (2002). Comparison of potato amylopectin starches and potato starches-influence of year and variety. Carbohydrate Polymers, 47, 331-340.

Swinkels, J. J. M. (1985). Composition and properties of commercial native starches. Starch, $37,1-5$.

Tabata, S. and Hizukuri, S (1971). Studies on starch phosphate. 2. Starch, 23, 267-272. 
Tahir, R., Ellis, P. R. and Butterworth, P. J. (2010). The relation of physical properties of native starch granules to the kinetics of amylolysis catalysed by porcine pancreatic $\alpha$-amylase. Carbohydrate Polymers, 81, 57-62.

Takase, K., Matsumoto, T., Mizuno, H. and Yamane, K. (1992). Site-directed mutagenesis of active site residues in Bacillus subtilis a-amylase. Biochimica Biophysica Acta, 1]20, 281-288.

Takeda Y. and Hizukuri, S. (1981). Re-examination of the action of sweet potato beta amylase on phosphorylated (1-4) a-glucan. Carbohydrate Research, 89, 174-178.

Takeda Y. and Hizukuri, S. (1982). Location of phosphate groups in potato amylopectin. Carbohydrate Research, 102, 321-327.

Takeda, Y., Hizukuri, S., Takeda, C, and Suzuki, A. (1987). Structures of branched molecules of amyloses of various origins and molar fractions of branched and unbranched molecules. Carbohydrate Research, 165, 139-145.

Tamaki, S., Hisamatsu, M., Teranishi, K., Adachi, T. and Yamada, T. (1998). Structural Change of maize starch granules by ball-mill treatment. Starch, 50,342-348.

Tang, M. C. and Copeland, L. (2007). Analysis of complexes between lipids and wheat starch. Carbohydrate Polymers, 67, 80-85.

Tao, B. Y, Reilly, P. J. and Robyt, J. F. (1989). Detection of covalent intermediate in the mechanism of action of porcine pancreatic a-amylase by using $13 \mathrm{C}$ nuclear magnetic resonance. Biochimica et Biophysica Acta, 995, 214-220.

Tatge, H., Marshall, J., Martin, C., Edwards, E. A. and Smith, A. M. (1999). Evidence that amylose synthesis occurs within the matrix of starch granules. Plant Cell Environment, 22, 543-550.

Tester, R. F, and Karkalas, J. (1996). Swelling and gelatinization of oat starches, Cereal Chemistry, 73, 271-273. 
Tester, R. F. and Karkalas, J. (2002). Starch In A. Steinbüchel (series Ed), E. J. Vandamme, S. De Baets, and A. Steinbüchel (volume Eds), Biopolymers Volume 6, Polysaccharides II: Polysaccharides from Eukaryotes (pp. 381-438). Wiley-VCH, Weinheim.

Tester, R. F, and Morrison, W. R. (1990a). Swelling and gelatinization of cereal starches. 1. Effects of amylopectin, amylose, and lipids, Cereal Chemistry, 67, 551-557.

Tester, R. F., Karkalas, J. and Qi, X. (2004). Starch - composition, fine structure and architecture. Journal of Cereal Science, 39, 151-165.

Tester, R.F. and Morrison, W, R. (1990b). Swelling and gelatinization of cereal starches II. Waxy rice starches. Cereal Chemistry, 67, 558-563.

Thoma, J. A. and French, D. (1960). The starch-iodine-iodide interaction. Part I. Spectrophotometric investigations. Journal of the American Chemical Society, 82, 41444147.

Thompson, D. B. (2000). On the non-random nature of amylopectin branching. Carbohydrate Polymers, 43, 223-239.

van Soest, J. J. G., Tournois, H., de Wit, D., Johannes, F.G, and Vliegenthart, J. F. G. (1995). Short-range structure in (partially) crystalline potato starch determined with attenuated total reflectance Fourier-transform IR spectroscopy. Carbohydrate Research, 279, 201214.

Vandeputte, G. E. and Delcour, J. A. (2004). From sucrose to starch granule to starch physical behavior: A focus on rice starch. Carbohydrate Polymers, 58, 245-266.

Vandeputte, G. E., Derycke, V., Geeroms, J., and Delcour, J. A. (2003). Rice starches. II. Structural aspects provide insight into swelling and pasting properties. Journal of Cereal Science, 38, 53-59.

Vasanthan, T. and Hoover, R. (1992). A comparative study of the composition of lipids associated with starch granules from various botanical sources. Food Chemistry, 43, 1927. 
Vermeylen, R., Goderis, B. and Delcour, J. A. (2006). An X-ray study of hydrothermally treated potato starch. Carbohydrate Polymers, 64, 364-375.

Vermeylen, R., Goderis, B., Reynaers, H. and Delcour, J. A. (2004). Amylopectin molecular structure reflected in macromolecular organization of granular starch. Biomarcomolecules, 5, 1775-1786.

Villareal, C. P., Juliano, B. O. and Banos, L. (1986). Waxy gene factor and residual protein of rice starch granules. Starch, 38, 118-119.

Virtanen, T., Autio, K., Suortti, T., and Poutanen, K. (1993). Heat-induced changes in native and acid-modified oat starch paste. Joturnal of Cereal Science, 17, 137-145

Visser, R. G. F., Somhorst, I., Kuipers, G. J., Ruys, N. J., Feenstra, W. J. and Jacobsen, E. (1991). Inhibition of the expression of the gene for granule-bound starch synthase in potato by antisense constructs. Molecular Genetics and Genomics, 225, 289-296.

Visser, R. G. F., Suurs, L. C. J. M., Steeneken, P. A. M. and Jacobsen, E. (1997). Some physicochemical properties of amylose-free potato starch. Starch, 49, 443-448.

Waduge, R. N., Hoover, R., Vasanthan, T., Gao, J. and Li, J. (2006). Effect of annealing on the structure and physicochemical properties of barley starches of varying amylose content. Food Research International, 39, 59-77.

Waduge, R. N., Xu, S. and Seetharaman, K. (2010). Iodine absorption properties and its effect on the crystallinity of developing granules. Carbohydrate Polymers, doi:10.1016/J.Carb. Pol.2010.05.093.

Waigh, T. A., Gidley, M. J., Komanshek B. U. and Donald, A. M. (2000b). The phase transformations in starch during gelatinisation: a liquid crystalline approach. Carbohydrate Research, 328, 165-176.

Waigh, T. A., Kato, K. L., Donald, A. M., Gidley, M. J., Clarke, C. J. and Riekel, C. (2000a). Side-chain liquid-crystalline model for starch. Starch, 52, 450-460. 
Waigh, T. A., Perry, A. P., Riekel, C., Gidley, M. J. and Donald, A.M. (1998). Chiral side-chain liquid-crystalline polymeric properties of starch. Macromolecules, 31, 7980-7984.

Wakelin, J. H., Virgin, H. S. and Crystal, E. (1959), Development and comparison of two X-ray methods for determining the crystallinity of cotton cellulose. Journal of Applied Physics, $30,1654-1662$.

Wang, L. and Seib, P. A. (1996). Australian salt-noodle flours and their starches compared to US wheat flours and their starches. Cereal Chemistry, 73, 167-175.

Wang, L. Z. and White, P. J. (1994). Structure and properties of amylose, amylopectin and intermediate material of oat starches. Cereal Chemistry, 71, 263-268.

Wang, S. J., Yu, J. L., Gao, W. Y., Pang, J. P. and Liu, H. Y. (2007). Particle structural changes in native Chinese Yam (Dioscorea opposite Thunb var. Anguo) starches during acid hydrolysis. Carbohydrate Polymers, 69, 286-292.

Wang, S. J., Yu, J. L., Yu, J. G., Chen, H. X. and Pang, J. P. (2007). The effect of acid hydrolysis on morphological and crystalline properties of Rhizoma Dioscorea starch. Food Hydrocolloids, 21, 1217-1222.

Wang, Y.-J., White, P., Pollak, L, and Jane, J. (1993). Amylopectin and intermediate materials in starches from mutant genotypes of the Oh43 inbred line. Cereal Chemistry, 70, 521525.

Watanabe, T. and French, D. (1980). Structural features of nageli amylodextrin as indicated by enzymic degradation. Carbohydrate Research, 84, 115-123.

Watcharatewinkul, Y., Puttanlek, C., Rungsardthong, V. and Uttapap, D. (2009). Pasting properties of a heat-moisture treated canna starch in relation to its structural characteristics. Carbohydrate Polymers, 75, 505-511.

Watcharatewinkul, Y., Uttapap, D., Puttanlek, C. and Rungsardthong, V. (2010). Enzyme digestibility and acid/shear stability of heat-moisture treated canna starch. Starch, 62, 205-216. 
Watson, S. A. (1964). Determination of starch gelatinization temperature. In R. L. Whistler, R. J. Smith, M. L. Wolfrim (Eds). Methods in Carbohydrate Chemistry, (pp 286-288). Academic Press, NY.

Wei, C., Qin, F., Zhu, L., Zhou, W., Chen, Y., Wang, Y., Gu, M. and Liu, Q. (2010). Microstructure and ultrastructure of high-amylose rice resistant starch granules modified by antisense RNA inhibition of starch branching enzyme. Journal of Agricultural Food Chemistry, 58, 1224-1232.

Whistler, R. L. and BeMiller, J. N. (1996). Starch. In: R. L. Whistler and J. N. BeMiller (Eds), Carbohydrate Chemistry for Food Scientists, (pp. 117-151). Eagan Press, St. Paul, MN.

Whistler, R. L. and BeMiller, J. N. (1997). Starch. In R. L. Whistler and J. N. Be-Miller (Eds), Carbohydrate Chemistry for Food Scientists, (pp 117-151). American Association of Cereal Chemists. St Paul.

White, P. J., Abbas, I. R. and Johnson, L. J. (1989). Freeze-thaw stability and refrigeratedstorage retrogradation of starches. Starch, 4I, 176-180.

Widya, Y., Gunawan, N., BeMiller, J. N. (2010). Methods for determining relative average number of channels per maize starch granule and digestion of raw granules of mutant maize cultivars by amyloglucosidase. Cereal Chemistry, 87, 194-203.

Withers, S. G. and Aebersold, R. (1995). Approaches to labeling and identification of active-site residues in glycosidases. Protein Science, 4, 361-372.

Wolever, T. M. S. and Mehling, C. (2002). High-carbohydrate-low glycemic index dietary advice improves glucose disposition index in subjects with impaired glucose tolerance. British Journal of Nutrition, 87, 477-487.

Wongsagonsup, R., Varavinit, S. and James BeMiller, N. (2009). Increasing slowly digestible starch content of normal and waxy maize starches and properties of starch products. Cereal Chemistry, 85, 738-745.

Wu, H. C. H. and Sarko, A. (1978a). The double helical molecular structure of crystalline Bamylose. Carbohydrate Research, 61, 7-25. 
Wu, H. C. H., and Sarko, A. (1978b). The double helical molecular structure of crystalline Aamylose. Carbohydrate Research, 61, 27-40.

Yamamoto, M. Sano, T. and Yasunaga, T. (1982). Interaction of amylose with iodine. I. Characterization of cooperativity binding isotherms for amyloses. Bulletin of the Chemical Society of Japan, 55, 1886-1889.

Yamashita, Y. and Monobe, K. (1971). Single crystals of amylose V complexes. 3. Crystals with 81 helical configuration. Journal of Polymer Science Part B: Polymer Physics, 9, 1471.

Yashushi, Y. B., Takenouchi, T. and Takeda, Y. (2002). Molecular structure and some physicochemical properties of waxy and low-amylose barley starches. Carbohydrate Polymers, 47, 159-167.

Yoon, J.-W., Lim, S.-T. (2003). Molecular fractionation of starch by density-gradient ultracentrifugation. Carbohydrate Research, 338, 611-617.

Yoshimoto, Y., Tashiro, J., Takenouchi, T. and Takeda, Y. (2000). Molecular structure and some physicochemical properties of high-amylose barley starch. Cereal Chemistry, 77, 279-285.

Yoshino, Y., Hayashi, M. and Seguchi, M. (2005). Presence and Amounts of Starch Granule Surface Proteins in Various Starches. Cereal Chemistry, 82, 739-742.

Yoshino, Z., Komaki, T., and Kurahashi, Y. 1994. Process for producing HMT starch. US. patent 5,362329 .

Yu, X., Houtman, C. and Atalla, R. H. (1996). The complex of amylose and iodine. Carbohydrate Research, 292, 129-141.

Yuan, R. C., Thompson, D. B. and Boyer, C. D. (1993). Fine structure of amylopectin in relation to gelatinization and retrogradation behaviour of maize starches from three wax-containing genotypes in two inbred lines. Cereal Chemistry, 70, 81-89.

Yuryev, V. P., Krivandin, A. V., Kiseleva, V. I., Wasserman, L. A., Genkina, N. K. and Fornal, J., Blaszczak, W. and Schiraldi, A. (2004). Structural parameters of amylopectin clusters 
and semi-crystalline growth rings in wheat starches with different amylose content. Carbohydrate Research, 339, 2683-2691.

Yuryev, V. P., Kozlov, S. S., Noda, T., Bertoft, E. and Blennow, A. (2007). Influence of different GBSS1 and GWD combinations on the amylose localization within wheat and potato starch granules. In V. P. Yuryev, P. Tomasik and E. Bertoft, (Eds), Starch: Achievements in Under-standing of Structure (pp. 1-47). Nova Science, NY.

Yusuph, M., Tester, R. F., Ansell, R. and Snape, C. E. (2003). Composition and properties of starches extracted from tubers of different potato varieties grown under the same environmental conditions. Food Chemistry, 82, 283-289.

Zavareze, E. R., Storck, C. R., Castro, L. A. S., Schirmer, M. A. and Dias, A. R. G. (2010), Effect of heat-moisture treatment on rice starch of varying amylose content. Food Chemistry, 121, 358-365

Zhang, G., Ao, Z. and Hamaker, B. R. (2006). Slow digestion property of native cereal starches. Biomacromolecules, 7, 3252-3258.

Zhang, T. and Oates, C. G. (1999). Relationship between $\alpha$-amylase degradation and physicochemical properties of sweet potato starches. Food Chemistry, 65, 157-163.

Zhou, Y., Hoover, R. and Liu, Q. (2004). Relationship between $\alpha$-amylase degradation and the structure and physicochemical properties of legume starches. Carbohydrate Polymers, 57, 299-317.

Ziegler, G. R., Creek, J. A. and Runt, J. (2005). Spherulitic crystallization in starch as a model for starch granule initiation. Biomacromolecules, 6, 1547-1554.

Ziegler, G. R., Thompson, D. B. and Casasnovas, J. (1993). Dynamic measurement of starch granule swelling during gelatinisation. Cereal Chemistry, 70, 247-261.

Zobel, H. F. (1988a). Molecules to granules: A comprehensive starch review. Starch, 40, 44 50. 
Zobel, H. F., (1988b). Starch crystal transformations and their industrial importance. Starch, 40, 1-7. 


\section{PUBLICATIONS}

Varatharajan, V., Hoover, R, Liu, Q., Seetharaman, K. (2010). The impact of heat-moisture treatment on the molecular structure and physicochemical properties of normal and waxy potato starches. Carbohydrate Polymers, 81, 466-475.

Varatharajan, V., Hoover, R., Jihong, L., Vasanthan, T., Nantanga K. K. M., Seetharaman, K., Liu, Q., Donner, E., Jaiswal, S. and Chibbar, R. N. (2010). Impact of structural changes at different temperatures of heat-moisture treatment on the susceptibility of normal and waxy potato starches towards hydrolysis by porcine pancreatic alpha amylase. (In-review- Food Chemistry). 


\section{SCHOLARSHIPS AND AWARDS}

- School of Graduate Studies (SGS) Merit Award (2008), Memorial University of Newfoundland

- First place in the Graduate Student Competition (Carbohydrate Division) at the Institute of Food Technologists' (IFT) Conference held in Anaheim, CA, June 6-9, 2009

- Barrowman Biochemistry Travel Award for 2009, Memorial University of Newfoundland

- Fellow of the School of Graduate Studies (2009-2010), Memorial University of Newfoundland

- AACC International Student Travel Award (2010), AACC International Annual Meeting in Savannah GA, USA (Oct-24-27, 2010)

- University of Guelph/Ontario Ministry of Agriculture, Food and Rural Affairs (OMAFRA)/ High Quality Personnel (HQP) Scholarship (2010-2011)

- University International Graduate Scholarship (UIGS) (2010-2011), University of Guelph 
APPENDICES 
Appendix 1

Curve fitting for XRD using Gaussian function 

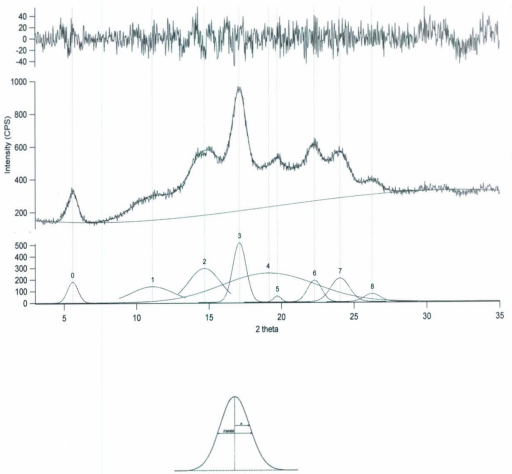

- Peak height and FWHM were calculated after subtracting the amorphous (peak 4) background 
Appendix 2

Standard curve for determination of amylose content (Hoover and Ratnayake, 2004) 


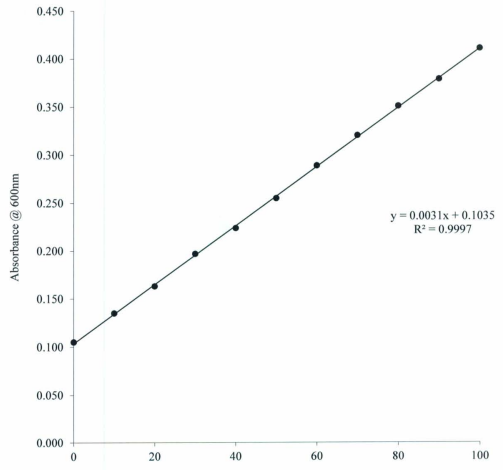

Amylose percentage (\%) 


\section{Appendix 3}

Standard curve for determination of reducing sugar as maltose (Bruner, 1964) 


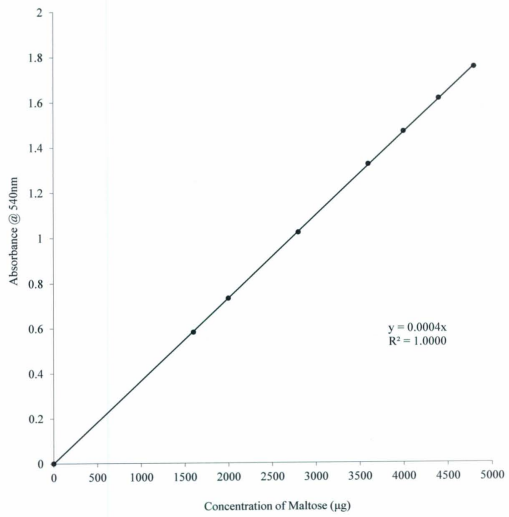


Appendix 4

Phosphorus calibration curve (Morrison, 1964) 


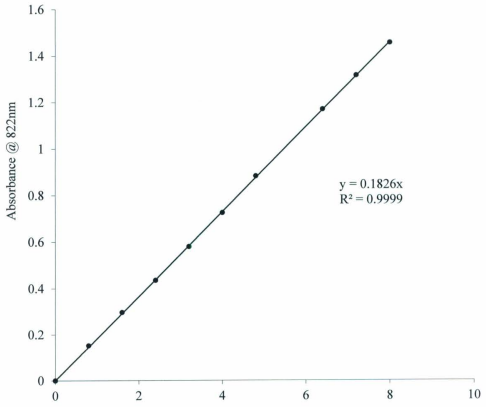

Phosphorus Concentration $(\mu \mathrm{g})$ 



